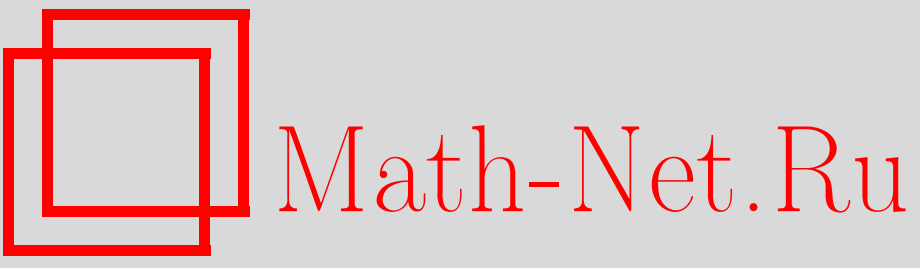

Ю. М. Мовсисян, Сверхтождества в алгебрах и многообразиях, УМН, 1998, том 53, выпуск 1, 61-114

DOI: https://doi.org/10.4213/rm9

Использование Общероссийского математического портала Math-Net.Ru подразумевает, что вы прочитали и согласны с пользовательским соглашением

http://www.mathnet.ru/rus/agreement

Параметры загрузки:

IP : 35.173 .219 .12

26 апреля 2023 г., 12:31:55 


\section{СВЕРХТОЖДЕСТВА В АЛГЕБРАХ И МНОГООБРАЗИЯХ}

Ю. М. Мовсисян

\section{СОДЕРЖАНИЕ}

Введение

$\S 1$. Понятие сверхтождества. Сверхтождества бинарных представлений 64

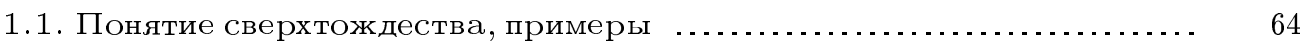

1.2. Бинарные теоремы Кэли для полугруп, идемпотентных и коммутативных полугрупп, групп и мультипликативных групп полей ..... 72

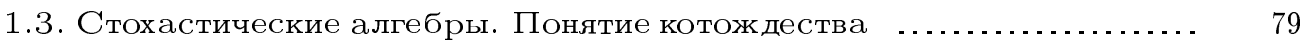

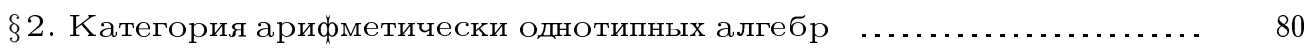

2.1. Гомоморфизмы и автоморфизмы арифметически однотипных алгебр 80

2.2. Сверхмногообразия алгебр …............................. 85

$\S 3$. Категория арифметически однотипных систем. Неклассическая се-

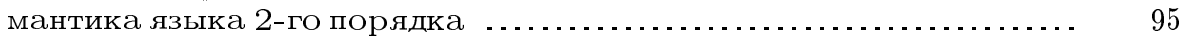

$\S 4$. Нетривиальные сверхтождества ассоциативности и дистрибутивности в обратимых и близких к ним алгебрах .................. 98

Список литературы .................................................... 112

\section{Введение}

Теория моделей (алгебр) изучает связи между формальным языком и его интерпретациями или моделями (алгебрами). Простейший из формальных языков - язык первого порядка (ступени) [1], [2]. Основателями теории моделей (алгебр) язька первого порядка были Левенгейм, Сколем, Гедель, Тарский, Мальцев, Биркгоф.

Следующим по важности формальным языком является язык второго порядка (второй ступени), подробно описьваемьй в [1], [2].

Формулы в язьке 2-го порядка (называемые формулами 2-го порядка) составляются из тех же логических символов, предметных, предикатных и функциональных переменных, что и в формальном язьке 1-го порядка. Отличие состоит лишш в том, что в формулах 2-го порядка кванторные символы $\forall, \exists$ могут связывать не только предметные переменные, но и предикатные или функциональные переменные.

Формула язька 2-го порядка назьвается замкнутой (козамкнутой), если каждая ее предметная (соответственно функциональная и предикатная) переменная связана квантором, т.е. замкнутая (козамкнутая) формула не содержит свободных предметных 
(соответственно функциональных и предикатных) переменных. Формула 2-го порядка назьвается абсолютно замкнутой, если каждая ее (предметная, функциональная, предикатная) переменная связана квантором, т.е. такая формула не содержит ни предметных, ни функциональных, ни предикатных свободных переменных.

О классах алгебр и алгебраических систем, определяемых формулами 2-го порядка общего вида, мало что известно [1], [3]-[5].

Важньй класс формул 2-го порядка со специализированньми кванторами выделен А. И. Мальцевьм [6], доказавшим, что для каждого свойства моделей, которое можно выразить квазиуниверсальными формулами, справедлива локальная теорема: если квазиуниверсальная формула $\Phi$ истинна на подсистемах $\mathscr{A}_{i}$, локально покрывающих алгебраическую систему $\mathscr{A}$, то $\Phi$ истинна и на $\mathscr{A}$ (см. также [7]).

С начала шестидесятых годов в различных разделах алгебры и ее приложениях исследуются следуюшие два класса абсолютно замкнутых формул второго порядка (применительные к алгебрам) с ограниченными кванторами:

$$
\begin{gathered}
\forall X_{1}, \ldots, X_{k} \exists X_{k+1}, \ldots, X_{m} \forall x_{1}, \ldots, x_{n}\left(W_{1}=W_{2}\right), \\
\forall X_{1}, \ldots, X_{m} \forall x_{1}, \ldots, x_{n}\left(W_{1}=W_{2}\right),
\end{gathered}
$$

где $W_{1}, W_{2}$ - термы (слова) от функциональных переменных $X_{1}, \ldots, X_{m}$ и от предметных переменных $x_{1}, \ldots, x_{n}$. Первая формула назьвается $\forall \exists(\forall)$-тождеством, а вторая - сверхтождеством или $\forall(\forall)$-тождеством. Вьполнимость (истинность) этих формул в алгебре $\mathscr{A}=(Q ; \Sigma)$ понимается с ограниченными функциональными кванторами $\left(\forall X_{i}\right),\left(\exists X_{j}\right)$, означающими: “для каждого значения $X_{i}=A_{i} \in \Sigma$ соответствующей арности", “сушествует значение $X_{j}=A_{j} \in \Sigma$ соответствуюшей арности"; предполагается возможность такой замены, т.е.

$$
\left\{\left|X_{1}\right|, \ldots,\left|X_{m}\right|\right\} \subseteq\{|A| \mid A \in \Sigma\}=T_{\mathscr{A}}
$$

где $|S|-$ арность $S$.

Алгебра $\mathscr{A}=(Q ; \Sigma)$ назьвается алгеброй с арифметическим типом $T \subseteq \mathbb{N}$ или $T$-алгеброй, если $T=T_{\mathscr{A}}$. Например, арифметический тип кольца $Q(+, \cdot)$ есть $\{2\}$.

Формула языка 2-го порядка (2-й ступени) без предикатных переменных называется $T$-формулой, если арность каждой ее функщиональной переменной содержится в $T$. Значение и истинность $T$-формулы в $T$-алгебре $\mathscr{A}=(Q ; \Sigma)$ определяем с такими же ограниченными функциональньми кванторами $\left(\forall X_{i}\right),\left(\exists X_{j}\right)$, что и в $\forall \exists(\forall)$-тождествах и сверхтождествах. При интерпретации $T$-формулы в $T$-алгебре $\mathscr{A}=(Q ; \Sigma)$ значения (соответствующих арностей) для свободных функщиональных переменных также берем из множества операций $\Sigma$.

Совокупность $T$-формул $\mathscr{F}$ назьвается выполнимой в $T$-алгебре $\mathscr{A}$, если существуют такие значения из $\mathscr{A}$ для всех входящих в элементы $\mathscr{F}$ свободных (предметных и функциональных) переменных, при которых формулы из $\mathscr{F}$ становятся истинньми в $\mathscr{A}$. Совокупность $T$-формул назьвается вьполнимой, если существует $T$-алгебра, в которой выполнима эта совокупность.

Пусть $n \in \mathbb{N}$. Совокупность $T$-формул $\Gamma$ назьвается $n$-вьполнимой, если сушествует $T$-алгебра, в которой вьполнимо любое подмножество $\mathscr{F} \subseteq \Gamma$ порядка $\leqslant n$. 
Указанная семантика согласована с гомоморфизмами $T$-алгебр [8], [9], определяемыми как пары $(\varphi, \widetilde{\psi})$ отображений с условием:

$$
\varphi A\left(x_{1}, \ldots, x_{n}\right)=[\widetilde{\psi}(A)]\left(\varphi x_{1}, \ldots, \varphi x_{n}\right) .
$$

Существование фильтрованных и ультрапроизведений, в соответствующей категории $T$-алгебр $(Q ; \Sigma)$ и их гомоморфизмов $(\varphi, \widetilde{\psi})$ (в качестве морфизмов), приводит к следующей теореме компактности для $T$-формул: если вьполнима ( $n$-вьполнима) каждая конечная часть бесконечной совокупности $T$-формул, то вьполнима $(n$-вьполнима) и вся заданная совокупность $T$-формул $(n=1,2, \ldots)$.

В качестве следствия получаем теорему компактности для абсолютно замкнутых $T$-формул (называемых $T$-предложениями). Поэтому, если $T$-предложение (в частности, $T$-сверхтождество) $F$ равносильно бесконечной системе $T$-предложений $\left(T\right.$-сверхтождеств) $\Gamma$, тогда существует конечное подмножество $\Gamma_{0} \subseteq \Gamma$, равносильное $F$.

Подобный подход к моделям и алгебраическим системам приводит к теореме компактности для формул 2-го порядка с ограниченньми функциональными и предикатньми кванторами (см. $\S 3$ ниже). Ограниченность предикатных кванторов $\left(\forall P_{i}\right),\left(\exists P_{j}\right)$ в моделях (алгебраических системах) здесь определяется аналогично ограниченности функциональных кванторов в алгебрах. ${ }^{1}$

Следующие результаты также показывают естественность формул вида (0.1) и (0.2) и их моделей (т.е. алгебр с такими формулами).

Хорошо известно, что любое полугрупповое слово с помошю тождества ассоциативности приводится к левонормированной форме расстановки скобок. Если же в слове участвуют различные, например, бинарные операции из алгебры $(Q ; \Sigma)$, тогда любое такое слово с помощью выполняющегося в этой алгебре $\forall \exists(\forall)$-тождества ассоциативности

$$
\forall X, Y \exists X^{\prime}, Y^{\prime} \forall x, y, z\left(X(x, Y(y, z))=X^{\prime}\left(Y^{\prime}(x, y), z\right)\right)
$$

приводится к левонормированной форме расстановки скобок, что легко доказывается индукцией по числу предметных переменных. При этом исходное слово может содержать только одну неассоциативную (например, квазигрупповую) операцию.

Аналогично, исходя из следуюшего $\forall \exists(\forall)$-тождества ассоциативности

$$
\forall X, Y \exists X^{\prime}, Y^{\prime} \forall x, y, z\left(X(Y(x, y), z)=X^{\prime}\left(x, Y^{\prime}(y, z)\right)\right),
$$

любое слово с бинарными операциями можно привести к правонормированной форме расстановки скобок. В частности, вместо $\forall \exists(\forall)$-тождеств ассощиативности $(0.3),(0.4)$ здесь можно взять и $\forall(\forall)$-тождества ассощиативности, т.е. сверхтождества ассоциативности. Возникает естественная задача - описание класса алгебр с формулами 2-го порядка (0.3), (0.4) (см. [10], [11]) или со сверхтождествами ассоциативности. О важности такой постановки задачи, например, в теории кордирований см. [12], [13]. Как

\footnotetext{
${ }^{1}$ Традиционная семантика формул 2-го порядка, определяемая на множествах (см., например, [2]), является частным случаем введенной семантики, если вместе с множеством рассматривать всевозможные операции и предикаты, определенные на нем.
} 
следствие изучения таких формул, возникает естественная характеристика бесконечных неассоциативных луп [11]: неассоциативная лупа $Q($ о) будет бесконечной тогда и только тогда, когда функциональное уравнение $x \circ(y \circ z)=A[B(x, y), z]$ имеет решение в множестве $Q$.

Каждая группа (полугрупп) вкладывается в симметрическую групу (полугруппу), тождества которой равносильны сверхтождествам; каждая алгебра вкладывается в алгебру функций, тождества которой равносильны сверхтождествам или $\forall \exists(\forall)$-тождествам (А. Кэли, П. Кон) ${ }^{2}$

Многообразия алгебр характеризуются (обычными) тождествами, однако многообразия многообразий (определяемые как абстрактные классы многообразий алгебр, замкнутых по подмногообразиям, по произведениям многообразий и по редуктам многообразий) уже характеризуются сверхтождествами [14] (см. также [9], где имеется характеризация и квазимногообразий многообразий).

Многообразия многообразий как класс многообразий алгебр, удовлетворяюший условиям биркгофовского типа, возникли в связи с мальцевскими классами многообразий $[15]$.

Отметим ряд результатов статьи.

В $\S 1$ полугруппы, групш и мультипликативные группы полей представляются (характеризуются) как полугрупшы и группы бинарных функций со сверхтождествами. Здесь применяется метод дважды транзитивных групп (ср. [18]).

В $\S 2$ доказывается теорема о представимости пар груп $(G, H)$, где $H \unlhd G$ в качестве пар групп автоморфизмов $(\varphi, \widetilde{\psi})$ и $(\varphi, \widetilde{\varepsilon})$ (где $\widetilde{\varepsilon}$ - тождественное отображение) одной и той же алгебры (cp. [19], [20]).

В $\S 2$ доказьваются также характеристики подпрямо неразложимых алгебр рассматриваемых сверхмногообразий и с их помошью характеризуются сверхтождества некоторых классических многообразий (ср. [21], [22]).

Параграф 4 посвящен нетривиальным сверхтождествам ассоциативности и дистрибутивности в обратимых и близких к ним алгебрах. В частности, характеризуются обратимые алгебры с нетривиальньм сверхтождеством левой (правой) дистрибутивности при наличии тривиального сверхтождества правой (левой) дистрибутивности. Важную роль здесь играет классическая теорема Муфанг [23].

Выражаю признательность профессору Г. М. Бергману из Калифорнийского университета (Беркли), предложившему ряд сокрашений в доказательствах некоторых результатов статьи.

\section{§ 1. Понятие сверхтождества. Сверхтождества бинарных представлений}

\section{1. Понятие сверхтождества, примеры}

Сверхтождество

$$
\forall X_{1}, \ldots, X_{m} \forall x_{1}, \ldots, x_{n}\left(W_{1}=W_{2}\right)
$$

\footnotetext{
${ }^{2}$ Примечательно также, что более ста лет назад в трудах известного немецкого математика Эрнста Шрёдера [16], [17] тождества рассмотрены как "уравнения", при этом "неизвестными" в них считались операции.
} 
для краткости записывается без кванторной приставки: $W_{1}=W_{2}$. Число $m$ называется функциональным рангом, а число $n$ - предметным рангом сверхтождества (1.0). Пусть $T \subseteq \mathbb{N}, T \neq \varnothing$. Сверхтождество (1.0) называется $T$-сверхтождеством, если $\left\{\left|X_{1}\right|, \ldots,\left|X_{m}\right|\right\} \subseteq T . T$-алгебра - это алгебра с арифметическим типом $T$ (см. введение).

Будем говорить, что в $T$-алгебре $\mathscr{A}=(Q ; \Sigma)$ вьполняется $T$-сверхтождество (1.0), если равенство $W_{1}=W_{2}$ справедливо, когда в нем каждая предметная переменная и каждая функциональная переменная заменяются соответственно любым элементом из $Q$ и любой операцией соответствуюшей арности из $\Sigma$.

$T$-алгебра $\mathscr{A}=(Q ; \Sigma)$ назьвается функционально тривиальной, если она по каждой арности $n \in T$ содержит лишш одну $n$-арную операцию $A \in \Sigma$. В противном случае $T$-алгебра назьвается функционально нетривиальной. Функционально нетривиальные алгебры кратко будем назьвать нетривиальными алгебрами. В функщионально тривиальных алгебрах понятия сверхтождества и тождества неразличимы.

$T$-сверхтождество можно трактовать и как пару элементов алгебры слов (термов) с арифметическим типом $T$. Идея алгебры слов приводит к такой $T$-алгебре, множество $n$-арных операций которой $(n \in T)$ имеет сколь угодно большую мощность. Сперва определим арифметический тип для абстрактных множеств. Если $U-$ произвольное множество и $\Theta: U \rightarrow \mathbb{N}$ - некоторое отображение этого множества в множество всех натуральных чисел, то образ $\Theta(U) \subseteq \mathbb{N}$ будем назьвать арифметическим типом $U$. При этом, если $\omega \in U$ и $\Theta(\omega)=n$, то $\omega$ называется $n$-арным, а $n$ называется арностью элемента $\omega$ (обозначение: $n=|\omega|$ ). Множество с арифметическим типом $T \subseteq \mathbb{N}$ называется $T$-множеством. Далее стандартным путем определяется понятие алгебры слов с арифметическим типом $T$.

Пусть $\mathscr{X}$ - произвольное непустое множество, а $U$ - произвольное $T$-множество $(\mathscr{X} \cap U=\varnothing)$. Элементы множества $\mathscr{X}$ назовем предметными переменными, а Элементы множества $U$ - функциональными переменными. Понятие $T$-слова (или $T$-терма) определяется индуктивно: во-первых, каждое предметное переменное есть $T$-слово, и во-вторых, если $\omega \in U-$ функциональная переменная с арностью $m$ и $V_{1}, \ldots, V_{m}$ суть $T$-слова, то выражение $\omega\left(V_{1}, \ldots, V_{m}\right)$ также есть $T$-слово. Других $T$-слов нет. Равенство двух $T$-слов определяется их графическим совпадением. Обозначим через $(\mathscr{X}) U$ совокупность всех $T$-слов. Соответствуюшая $T$-алгебра $\langle(\mathscr{X}) U ; U\rangle=U(\mathscr{X})$ и есть $T$-алгебра слов. $T$-алгебра слов $U(\mathscr{X})$ назьвается стандартной, если множество $\mathscr{X}$ счетно, а множество $U$ для любого $n \in T$ содержит ровно счетное число $n$-арных операций.

Слово (терм) - это $T$-слово для некоторого $T \subseteq \mathbb{N}$.

Пусть $U(\mathscr{X})$ - стандартная $T$-алгебра слов и пусть $\mathscr{X}=\left\{x_{1}, \ldots, x_{n}, \ldots\right\}$. Естественно выделяется $n$-арный слой $T$-алгебры $U(\mathscr{X})$, состояший из $n$-арных $T$-слов $(T$-термов):

а) каждый элемент $x_{i}$, где $i=1, \ldots, n$, является $n$-арным $T$-словом;

б) если $W_{1}, \ldots, W_{m}$ являются $n$-арными $T$-словами, то для любого $m$-арного функционального переменного $X \in U, m \in T$, выражение $X\left(W_{1}, \ldots, W_{m}\right)$ также является $n$-арным $T$-словом;

в) $T$-слово называется $n$-арным, если оно является $n$-арным согласно а) и б). 
$n$-арное слово (терм) - это $n$-арное $T$-слово при некотором $T \subseteq \mathbb{N}$.

Класс алгебр называется классом $T$-алгебр, если каждая его алгебра есть $T$-алгебра. Алгебры $\mathscr{A}$ и $\mathscr{A}^{\prime}$ назьваются арифметически однотипными, если $T_{\mathscr{A}}=T_{\mathscr{A}^{\prime}}$. Таким образом, группоид и кольцо являются арифметически однотипными алгебрами.

Сверхтождество $W_{1}=W_{2}$ будет $T$-сверхтождеством, если $W_{1}, W_{2}$ являются элементами $T$-алгебры слов или $W_{1}, W_{2}$ являются $T$-словами.

Систему $T$-сверхтождеств считаем истинной (и говорим, что система выполняется) в $T$-алгебре $\mathscr{A}$, если в $\mathscr{A}$ вьполняется каждое сверхтождество этого семейства; $T$-сверхтождество $W_{1}=W_{2}$ выполняется в классе $T$-алгебр, если оно вьполняется в любой алгебре этого класса. В частности, можно говорить о сверхтождествах многообразий: сверхтождество $W_{1}=W_{2}$ называется сверхтождеством многообразия $V$, если оно выполняется в каждой алгебре $\mathscr{A} \in V$. Сверхтождество называется нетривиальным, если его функциональный ранг $>1$, и тривиальным - в противном случае.

Если многообразия $V_{1}$ и $V_{2}$ (с одной и той же сигнатурой) удовлетворяют некоторому сверхтождеству, то их решеточное объединение $V_{1} \vee V_{2}$ также удовлетворяет этому сверхтождеству.

Каждое тождество многообразия $V$ будет его сверхтождеством (разумеется, надо рассматривать символы операции тождества как функциональные переменные) тогда и только тогда, когда клон многообразия $V$ является $\varepsilon$-свободным в смысле [9]. В частности, каждое тождество многообразия $V$ будет сверхтождеством полиномиальной (термальной) алгебры $P(\mathscr{A})$ любой алгебры $\mathscr{A} \in V$ тогда и только тогда, когда клон многообразия $V$ является относительно свободным [24].

Многообразие $V$ назовем совершенным, если каждое тождество этого многообразия является и его сверхтождеством. Класс всех совершенных многообразий (с одной и той же сигнатурой) образует подрешетку в решетке всех многообразий (с данной сигнатурой).

Пусть $\mathscr{L}$ - некоторьй непустой набор тождеств с сигнатурой $\tau$, а $M_{\mathscr{L}}^{\tau}-$ класс всех алгебр с сигнатурой $\tau$, в которых каждое тождество из $\mathscr{L}$ вьполняется как сверхтождество. $M_{\mathscr{L}}^{\tau}$ будет многообразием для любого $\mathscr{L} \neq \varnothing$. Многообразие $V$ алгебр с сигнатурой $\tau$ будет совершенным тогда и только тогда, когда сушествует множество $\mathscr{L}$ тождеств с сигнатурой $\tau$ такое, что

$$
V=M_{\mathscr{L}}^{\tau}
$$

Каждое многообразие (или класс алгебр с одной и той же сигнатурой) $V$ содержится в наименьшем совершенном многообразии $\widehat{V}$ (назьваемом совершенной оболочкой $V$ ), определяемом всеми теми тождествами $V$, которые вьполняются в $V$ как сверхтождества. Например (это следует из теоремы $2.3, \S 2$ ), совершенная оболочка многообразия булевых алгебр определяется тождествами:

$$
\begin{gathered}
x+x=x, \quad x \cdot x=x, \\
x+y=y+x, \quad x \cdot y=y \cdot x, \\
x+(y+z)=(x+y)+z, \quad x \cdot(y \cdot z)=(x \cdot y) \cdot z, \\
x(y+z)=x y+x z, \quad x+y z=(x+y)(x+z),
\end{gathered}
$$




$$
\begin{gathered}
\left(x^{\prime}\right)^{\prime}=x, \\
x^{\prime}+y=(x+y)^{\prime}+y, \quad x^{\prime} \cdot y=(x \cdot y)^{\prime} \cdot y, \\
(x+y)^{\prime}+\left(x+y^{\prime}\right)^{\prime}=x^{\prime}, \quad(x \cdot y)^{\prime} \cdot\left(x \cdot y^{\prime}\right)^{\prime}=x^{\prime} .
\end{gathered}
$$

Структурная характеризация таких алгебр содержится в [21]. Частным случаем совершенных многообразий являются солидные многообразия [25]. Многообразие $V$ называется солидным, если каждое тождество этого многообразия является сверхтождеством не только для $V$, но и для полиномиальной алгебры $P(\mathscr{A})$ любой алгебры $\mathscr{A} \in V$. Класс всех солидных многообразий (с одной и той же сигнатурой) образует подрешетку в решетке всех совершенных многообразий (с данной сигнатурой).

$T$-сверх тождество $W_{1}=W_{2}$ назьвается следствием системы $T$-сверх тождеств $\mathscr{L}$, если всякий раз, когда система $\mathscr{L}$ вьполняется в $T$-алгебре, в ней выполняется и сверхтождество $W_{1}=W_{2}$, т.е. для любой $T$-алгебры $\mathscr{A}$

$$
\mathscr{A} \models \mathscr{L} \rightarrow \mathscr{A} \models\left(W_{1}=W_{2}\right)
$$

(запись $\mathscr{A} \models \mathscr{L}$ означает вьполнимость любого сверх тождества из $\mathscr{L}$ в алгебре $\mathscr{A}$ ). О соответствующей теореме о полноте для сверхтождеств см. [8], [9] и [21].

Сверхтождество $w_{1}=w_{2}$ назьвается сверхтождеством ассоциативности (абелевости, коммутативности, левой дистрибутивности, правой дистрибутивности, ...), если при игнорировании функциональных переменных сверхтождество $w_{1}=w_{2}$ преврашается в равенство ассоциативности (абелевости, коммутативности, левой дистрибутивности, правой дистрибутивности, ...), т.е. в равенство $((x, y), z)=(x,(y, z))$ (соответственно: $((x, y),(u, v))=((x, u),(y, v)),(x, y)=(y, x)$, $(x,(y, z))=((x, y),(x, z)),((x, y), z)=((x, z),(y, z)), \ldots)$.

ПримеР 1.0. В любом кольце $Q(+, \cdot)$ вьполняются сверхтождества

$$
\begin{aligned}
& X(X(Y(x, x), Y(x, x)), Y(X(x, x), X(x, x))) \\
& \quad=X(Y(X(x, x), X(x, x)), X(Y(x, x), Y(x, x))), \\
& \quad X(Y(Y(x, x), X(x, x)), Y(X(x, x), Y(x, x))) \\
& \quad=X(Y(X(x, x), Y(x, x)), Y(Y(x, x), X(x, x))) .
\end{aligned}
$$

Известно, что всякий группоид (в частности, и группоид слов) вкладывается в мультипликативную часть некоторого кольца. Поэтому, если в многообразии всех колец вьполняется сверхтождество $W_{1}=W_{2}$, тогда, заменяя все функциональные переменные в $W_{1}, W_{2}$ точкой, равенство $W_{1}=W_{2}$ превратим в графическое равенство, поскольку в группоиде слов вьполняются лишь тождества вида $W=W$.

В любом коммутативном кольце вьполняется сверхтождество коммутативности

$$
X(x, y)=X(y, x),
$$

а в любом ассоциативном кольце - сверхтождество ассоциативности

$$
X(x, X(y, z))=X(X(x, y), z) .
$$


ПримеР 1.1. В полиномиальной алгебре любой группы (полугруппы, лупы Муфанг) вьполняется сверхтождество

$$
X(Y(x, x), Y(x, x))=Y(X(x, x), X(x, x)) .
$$

В полиномиальной алгебре любой коммутативной групшы (полугрупшы) выполняется сверхтождество абелевости

$$
X(Y(x, y), Y(u, v))=Y(X(x, u), X(y, v)) .
$$

ПримеР 1.2. В любой решетке $Q(+, \cdot)$ выполняются тривиальные сверхтождества идемпотентности, коммутативности и ассоциативности:

$$
\begin{gathered}
X(x, x)=x \\
X(x, y)=X(y, x) \\
X(x, X(y, z))=X(X(x, y), z) .
\end{gathered}
$$

В нетривиальной решетке $Q(+, \cdot)$ не выполняется сверхтождество

$$
X(x, Y(x, y))=x
$$

однако в любой решетке $Q(+, \cdot)$ выполняются нетривиальные сверхтождества

$$
\begin{aligned}
Y(y, x) & =Y(y, X(x, Y(x, y))), \\
Y(X(x, z), y) & =Y(X(x, z), X(y, Y(y, z))) .
\end{aligned}
$$

Сверхтождество $W_{1}=W_{2}$ назьвается однородным (по А.И. Мальцеву), если в словах $W_{1}$ и $W_{2}$ участвуют одни и те же предметные переменные. Очевидно, что любое сверхтождество, вьполняюшееся в нетривиальной решетке, является однородным. Поэтому однородным является и любое сверхтождество любого нетривиального многообразия решеток.

ПримеР 1.3. Решетка будет модулярной тогда и только тогда, когда в ней вьполняется сверхтождество

$$
X(Y(x, X(y, z)), Y(y, z))=Y(X(x, Y(y, z)), X(y, z))
$$

Решетка будет дистрибутивной тогда и только тогда, когда в ней вьполняются следуюшие нетривиальные сверхтождества левой и (или) правой дистрибутивности:

$$
\begin{aligned}
& \left(d_{1}\right) \quad X(x, Y(y, z))=Y(X(x, y), X(x, z)), \\
& \left(d_{2}\right) \quad X(Y(x, y), z)=Y(X(x, z), X(y, z)) .
\end{aligned}
$$

В полиномиальной алгебре любой дистрибутивной решетки, следовательно, и любой полурешетки вьполняются сверхтождества дистрибутивности $\left(d_{1}\right),\left(d_{2}\right)$. 
ПРимеР 1.4. В любой булевой алгебре $Q\left(+, \cdot,{ }^{\prime}\right)$ вьполняется следующее нетривиальное сверхтождество:

$$
X\left(Y(x, y)^{\prime}, z\right)^{\prime}=Y\left(X\left(x^{\prime}, z\right)^{\prime}, X\left(y^{\prime}, z\right)^{\prime}\right)
$$

(здесь штрих ' выступает в роли унарной функциональной переменной).

Множество всех бинарных операций, определенных на $Q$, обозначим через $\mathscr{F}_{Q}^{2}$, и на этом множестве определим следуюшие операции:

$$
\begin{aligned}
& A \cdot B(x, y)=A[x, B(x, y)], \\
& A \circ B(x, y)=A[B(x, y), y],
\end{aligned}
$$

где $A, B \in \mathscr{F}_{Q}^{2}, x, y \in Q$. Операции ( · ) и ( о ) назьваются правым и левьм умножением операций соответственно.

Множество $\mathscr{F}_{Q}^{2}$ образует моноид относительно левого и правого умножения операший. Эти две полугруппы изоморфины. Единичным элементом для полугруппы $\mathscr{F}_{Q}^{2}(\cdot)$ служит элемент $E \in \mathscr{F}_{Q}^{2}$, определенньй по правилу $E(x, y)=y$, а единичным элементом полугрупшы $\mathscr{F}_{Q}^{2}\left(\right.$ ( ) - элемент $F \in \mathscr{F}_{Q}^{2}$, определенньй по правилу $F(x, y)=x$. Изоморфизм между указанными моноидами устанавливается соответствием $A \rightarrow A^{*}$, где $A^{*}(x, y)=A(y, x)$. Действительно, оно биективно и $(A \cdot B)^{*}=A^{*} \circ B^{*}$, поскольку:

$$
(A \cdot B)^{*}(x, y)=A \cdot B(y, x)=A(y, B(y, x))=A^{*}\left(B^{*}(x, y), y\right)=A^{*} \circ B^{*}(x, y) .
$$

ПРЕДЛОЖЕНИЕ 1.0. В неодноэлементной бинарной алгебре $(Q ; \Sigma)$ с условием $E, F \in \Sigma$ (следовательно, и в полиномиальной алгебре любой неодноэлементной алгебрьи)

1) не выполняется тривиальное сверхтождество коммутативности;

2) не выполняется никакое нетривиальное сверхтождество ассоииативности.

Если в такой алгебре выполняется:

3) нетривиальное сверхтождество абелевости, тогда оно будет иметь функииональный ранг 2 и вид (1.1);

4) нетривиальное сверхтождество левой дистрибутивности, тогда оно будет иметь функииональныи й ранг 2 и вид $\left(d_{1}\right)$;

5) нетривиальное сверхтождество правой дистрибутивности, тогда оно будет иметь функциональный ранг 2 и вид $\left(d_{2}\right)$.

Доказательство предложения 1.0 проводится путем непосредственной проверки.

Бинарная операция $A$, определенная на множестве $Q$, назьвается обратимой справа (слева), если для любых $a, b \in Q$ уравнение $A(a, x)=b($ соответственно $A(y, a)=b)$ имеет единственное решение в $Q$. Для однозначно определенных решений $x, y \in Q$ приняты обозначения: $x=A^{-1}(a, b), y={ }^{-1} A(b, a)$.

Таким образом, для любых $a, b \in Q$ имеем

$$
A\left(a, A^{-1}(a, b)\right)=A^{-1}(a, A(a, b))=b,
$$


если $A$ - обратима справа, и

$$
A\left({ }^{-1} A(b, a), a\right)={ }^{-1} A(A(b, a), a)=b,
$$

если $A$ - обратима слева. Операция $A^{-1}$ (соответственно ${ }^{-1} A$ ) назьвается правой (левой) обратной для обратимой справа (слева) операции $A$. Легко заметить, что операция $A^{-1}$ (соответственно ${ }^{-1} A$ ) также обратима справа (слева), причем:

$$
\left(A^{-1}\right)^{-1}=A={ }^{-1}\left({ }^{-1} A\right), \quad\left(A^{-1}\right)^{*}={ }^{-1}\left(A^{*}\right), \quad\left({ }^{-1} A\right)^{*}=\left(A^{*}\right)^{-1} .
$$

Бинарная операция $A \in \mathscr{F}_{Q}^{2}$ назьвается обратимой, если она обратима справа и слева. В этом случае операция $A$ имеет правую и левую обратные и для них справедливы равенства

$$
\left({ }^{-1}\left(A^{-1}\right)\right)^{-1}={ }^{-1}\left(\left({ }^{-1} A\right)^{-1}\right)=A^{*} .
$$

Бинарная алгебра $(Q ; \Sigma)$ назьвается обратимой (справа, слева), если каждая операция $A \in \Sigma$ обратима (справа, слева). Обратимая алгебра с одной операцией - квазигруппа.

Каждая обратимая алгебра $(Q ; \Sigma)$ порождает еще пять обратимых алгебр: $\left(Q ; \Sigma^{-1}\right),\left(Q ;{ }^{-1} \Sigma\right),\left(Q ;{ }^{-1}\left(\Sigma^{-1}\right)\right),\left(Q ;\left({ }^{-1} \Sigma\right)^{-1}\right)$ и $\left(Q ; \Sigma^{*}\right)$, где

$$
\begin{aligned}
\Sigma^{-1} & =\left\{A^{-1} \mid A \in \Sigma\right\}, \\
{ }^{-1} \Sigma & =\left\{{ }^{-1} A \mid A \in \Sigma\right\}, \\
{ }^{-1}\left(\Sigma^{-1}\right) & =\left\{{ }^{-1}\left(A^{-1}\right) \mid A \in \Sigma\right\}, \\
\left({ }^{-1} \Sigma\right)^{-1} & =\left\{\left(^{-1} A\right)^{-1} \mid A \in \Sigma\right\}, \\
\Sigma^{*} & =\left\{A^{*} \mid A \in \Sigma\right\} .
\end{aligned}
$$

При этом множество $\Sigma^{-1}$ (или $\left.{ }^{-1} \Sigma\right)$ содержательно и для обратимой справа (слева) алгебры $(Q ; \Sigma)$.

ПРЕДЛОЖЕНИЕ 1.1. Множество всех обратимых справа (слева) операций, определенных на $Q$, является группой относительно правого (левого) умноэения операций. Эти две группы изоморфны.

ДокАЗАТЕЛЬство. Множество всех обратимых справа (слева) операций, определенных на $Q$, обозначим через $\mathscr{F}_{Q}^{r}$ (соответственно $\left.\mathscr{F}_{Q}^{l}\right)$. Если $A, B \in \mathscr{F}_{Q}^{r}$, то и $A \cdot B \in \mathscr{F}_{Q}^{r}$, поскольку уравнение $A \cdot B(a, x)=A(a, B(a, x))=b$ имеет единственное решение $x \in Q$ для любых $a, b \in Q$. Для каждой обратимой справа операции $A \in \mathscr{F}_{Q}^{r}$ имеет место равенство

$$
A \cdot A^{-1}=A^{-1} \cdot A=E,
$$

где $A^{-1}$ - правая обратная операция для $A$.

Аналогично, совокупность $\mathscr{F}_{Q}^{l}$ всех обратимых слева операций, определенных на $Q$, образует группу относительно левого умножения операций. Соответствие $A \rightarrow A^{*}$ - изоморфизм между этими группами.

Если $\Sigma \subseteq \mathscr{F}_{Q}^{r}$, то через $(\Sigma)$ обозначим подгруппу группы $\mathscr{F}_{Q}^{r}(\cdot)$, порожденную подмножеством $\Sigma$. Если же $\Sigma \subseteq \mathscr{F}_{Q}^{l}$, тогда через $((\Sigma))$ обозначается подгруппа группы $\mathscr{F}_{Q}^{l}(\circ)$, порожденная $\Sigma$. 
ПРЕДЛОЖЕНИЕ 1.2. Если в обратимой справа алгебре $(Q ; \Sigma)$ выполняется сверхтождество $\left(d_{1}\right)$, тогда в алгебре $\left(Q ; \Sigma \cup \Sigma^{-1}\right)$ такэе выполняется сверхтождество $\left(d_{1}\right)$. Если в обратимой слева алгебре $(Q ; \Sigma)$ выполняется сверхтождество $\left(d_{2}\right)$, тогда в алгебре $\left(Q ;{ }^{-1} \Sigma \cup \Sigma\right)$ также выполняется это сверхтождество.

ДокАЗАТЕльство. Докажем первую часть предложения, аналогично доказьвается и вторая часть. Для любых $A, B \in \Sigma$ и для любых $x, y, z \in Q$ рассмотрим следуюшее равенство:

$$
A^{-1}\left(x, B\left(y, z^{\prime}\right)\right)=B\left(A^{-1}(x, y), A^{-1}(x, z)\right)
$$

(такой элемент $z^{\prime} \in Q$ существует ввиду обратимости справа рассматриваемых операций). Пользуясь определением правой обратной операции $A^{-1}$, имеем:

$$
\begin{aligned}
B\left(y, y^{\prime}\right) & =A\left(x, B\left(A^{-1}(x, y), A^{-1}(x, z)\right)\right) \\
& =B\left(A\left(x, A^{-1}(x, y)\right), A\left(x, A^{-1}(x, z)\right)\right)=B(y, z),
\end{aligned}
$$

откуда следует, что $z=z^{\prime}$. Таким образом, справедливо тождество

$$
A^{-1}(x, B(y, z))=B\left(A^{-1}(x, y), A^{-1}(x, z)\right)
$$

для любых $A, B \in \Sigma$. С помощью этого тождества теперь докажем тождество

$$
A^{-1}\left(x, B^{-1}(y, z)\right)=B^{-1}\left(A^{-1}(x, y), A^{-1}(x, z)\right) .
$$

Пусть

$$
A^{-1}\left(x, B^{-1}(y, z)\right)=B^{-1}\left(A^{-1}(x, y), A^{-1}\left(x, z^{\prime}\right)\right)
$$

тогда

$$
\begin{aligned}
A^{-1}\left(x, z^{\prime}\right) & =B\left(A^{-1}(x, y), A^{-1}\left(x, B^{-1}(y, z)\right)\right) \\
& =A^{-1}\left(x, B\left(y, B^{-1}(y, z)\right)\right)=A^{-1}(x, z) .
\end{aligned}
$$

Отсюда следует равенство $z=z^{\prime}$. Остается доказать тождество

$$
A\left(x, B^{-1}(y, z)\right)=B^{-1}(A(x, y), A(x, z))
$$

для любых $A, B \in \Sigma$. Пусть

$$
A\left(x, B^{-1}\left(y, z^{\prime}\right)\right)=B^{-1}(A(x, y), A(x, z)),
$$

тогда

$$
\begin{aligned}
B^{-1}\left(y, z^{\prime}\right) & =A^{-1}\left(x, B^{-1}(A(x, y), A(x, z))\right) \\
& =B^{-1}\left(A^{-1}(x, A(x, y)), A^{-1}(x, A(x, z))\right)=B^{-1}(y, z)
\end{aligned}
$$

и, следовательно, $z=z^{\prime}$. 
СлЕДСТВИЕ 1.1. Если в обратимой справа (слева) алгебре $(Q ; \Sigma)$ выполняется сверхтождество $\left(d_{1}\right)$ (соответственно $\left(d_{2}\right)$ ), тогда в расширенной алгебре $(Q ;(\Sigma))$ (соответственно $(Q ;((\Sigma)))$ ) также выполняется это сверхтождество.

ДокАЗАТЕЛЬство (первая часть). По индукции докажем справедливость тождества

$$
S(x, P(y, z))=P(S(x, y), S(x, z))
$$

для любых $S, P \in(\Sigma)$. Пусть

$$
\begin{array}{ll}
S=A_{1}^{\varepsilon_{1}} \cdots A_{m}^{\varepsilon_{m}}, & \varepsilon_{i}= \pm 1, \\
P=B_{1}^{\delta_{1}} \cdots B_{k}^{\delta_{k}}, & \delta_{j}= \pm 1,
\end{array}
$$

где $A_{i}, B_{j} \in \Sigma$. Длиной $\partial(S)$ операции $S \in(\Sigma)$ назовем число операций $A_{i} \in \Sigma$ в каком-нибудь представлении (1.3). Когда $\partial(S)=\partial(P)=1$, тогда $S, P \in \Sigma \cup \Sigma^{-1}$, и утверждение следует из предложения 1.2. Предположим справедливость утверждения в случае $\partial(S), \partial(P) \leqslant n-1$.

Далее возможны три случая:

1) $\partial(S)=n, \partial(P)<n$. Тогда $S=S_{1} \cdot S_{2}$, где $\partial\left(S_{1}\right), \partial\left(S_{2}\right)<n$, и по предположению индукции

$$
\begin{aligned}
S(x, P(y, z)) & =S_{1} \cdot S_{2}(x, P(y, z))=S_{1}\left(x, S_{2}(x, P(y, z))\right) \\
& =S_{1}\left(x, P\left(S_{2}(x, y), S_{2}(x, z)\right)\right)=P\left(S_{1}\left(x, S_{2}(x, y)\right), S_{1}\left(x, S_{2}(x, z)\right)\right) \\
& =P\left(S_{1} \cdot S_{2}(x, y), S_{1} \cdot S_{2}(x, z)\right)=P(S(x, y), S(x, z)) .
\end{aligned}
$$

2) $\partial(S)<n, \partial(P)=n$. Здесь полагается $P=P_{1} \cdot P_{2}$, где $\partial\left(P_{1}\right), \partial\left(P_{2}\right)<n$, и опять применяется индуктивное предположение.

3) $\partial(S)=\partial(P)=n$. Полагая $S=S_{1} \cdot S_{2}$, где $\partial\left(S_{1}\right), \partial\left(S_{2}\right)<n$, рассмотрение сводим к случаю 2).

\section{2. Бинарные теоремы Кэли для полугрупп, идемпотентных}

\section{и коммутативных полугрупп, групп и мультипликативных групп полей}

В дальнейшем, говоря о полугрупе $\mathscr{F}_{Q}^{2}$, мы имеем в виду полугрупу $\mathscr{F}_{Q}^{2}(\cdot)$. Эту полугруппу назовем полугруппой бинарных функций множества $Q$.

Пусть даны множество $Q$ и некоторая полугрупп $\Gamma$, вообще говоря, не связанная с $Q$. Будем говорить, что задано представление полугруппы $Г$ бинарными функциями множества $Q$ [18], если определено отображение

$$
(*): \Gamma \times Q^{2} \rightarrow Q
$$

- бинарное действие $\Gamma$ в $Q$, сопоставляюшее каждому $\gamma \in \Gamma$ и каждой паре $(x, y) \in Q^{2}$ элемент $\gamma *(x, y) \in Q$, причем предполагается справедливость условия:

$$
\gamma_{1} \gamma_{2} *(x, y)=\gamma_{1} *\left(x, \gamma_{2} *(x, y)\right)
$$

для любых $x, y \in Q$ и $\gamma_{1}, \gamma_{2} \in \Gamma$. 
Допустим, что задано такое представление. Тогда каждому $\gamma \in \Gamma$ отвечает некоторая бинарная операция $\varphi(\gamma) \in \mathscr{F}_{Q}^{2}$ :

$$
\varphi(\gamma)(x, y)=\gamma *(x, y) .
$$

Приходим к отображению $\varphi: \Gamma \rightarrow \mathscr{F}_{Q}^{2}$ с условием:

$$
\begin{aligned}
\varphi\left(\gamma_{1} \cdot \gamma_{2}\right)(x, y) & =\gamma_{1} \gamma_{2} *(x, y)=\gamma *\left(x_{1}, \gamma_{2} *(x, y)\right) \\
& =\varphi \gamma_{1}\left(x, \varphi \gamma_{2}(x, y)\right)=\left(\varphi \gamma_{1} \cdot \varphi \gamma_{2}\right)(x, y),
\end{aligned}
$$

т.е.

$$
\varphi\left(\gamma_{1} \cdot \gamma_{2}\right)=\varphi \gamma_{1} \cdot \varphi \gamma_{2}
$$

и отображение $\varphi$ - гомоморфизм из полугрупшы $\Gamma$ в полугрупу $\mathscr{F}_{Q}^{2}$. Таким образом, задавая представление $\Gamma$ бинарньми функциями множества $Q$, мы одновременно определяем гомоморфизм полугрупп

$$
\varphi: \Gamma \rightarrow \mathscr{F}_{Q}^{2} .
$$

Верно и обратное, если имеется некоторый гомоморфизм

$$
\varphi: \Gamma \rightarrow \mathscr{F}_{Q}^{2},
$$

тогда, полагая для любых $x, y \in Q$ и $\gamma \in \Gamma$

$$
\gamma *(x, y)=(\varphi \gamma)(x, y),
$$

простой проверкой убеждаемся, что здесь действительно имеем представление $\Gamma$ бинарными функциями множества $Q$. Итак, задание представления полугруппы $\Gamma$ бинарными функциями множества $Q$ равносильно заданию некоторого гомоморфизма $\Gamma$ в $\mathscr{F}_{Q}^{2}$. Такое представление полугруппы $\Gamma$ назовем ее бинарньм представлением в множестве $Q$.

Если задано бинарное представление полугруппы $\Gamma$, то элементы из $\Gamma$ действуют на множестве $Q$ как бинарные операции:

$$
\alpha(x, y)=\alpha *(x, y), \quad \alpha \in \Gamma, \quad x, y \in Q .
$$

Следовательно, множество $Q$ становится бинарной $\Gamma$-алгеброй с тождеством

$$
\alpha \cdot \beta(x, y)=\alpha(x, \beta(x, y)) \quad \forall \alpha, \beta \in \Gamma,
$$

называемой бинарным Г-полигоном.

Если эта Г-алгебра удовлетворяет сверхтождеству

$$
\left.W_{1}=W_{2} \text { (или формуле } \Phi\right),
$$

то будем говорить, что исходное представление удовлетворяет этому сверх тождеству (или формуле $\Phi$ ). 
Если $Г$ - моноид с единицей $e$, то в определении его бинарного представления добавляется условие $\varphi(e)=E$. Бинарное представление называется обратимым справа, если соответствующий бинарный $\Gamma$-полигон $Q$ обратим справа, т.е. для любого $\alpha \in \Gamma$ и для любых $a, b \in Q$ уравнение $\alpha(a, x)=b$ имеет единственное решение $x \in Q$. Аналогично определяется обратимость слева и обратимость бинарного представления. Нетрудно заметить, что любое бинарное представление любой групшы Г обратимо справа.

Бинарное представление полугрупшы называется точным, если соответствующий гомоморфизм есть мономорфизм.

ПРЕДЛОЖЕНИЕ 1.3 (бинарная теорема Кэли для моноидов). Каждый моноид Г имеет точное бинарное представление, удовлетворяющее сверхтождествам

$$
\begin{aligned}
X(x, X(y, z)) & =X(X(x, y), X(x, z)), \\
X(X(x, y), X(u, v)) & =X(X(x, u), X(y, v)) .
\end{aligned}
$$

ДокАЗАТЕльство. Пусть $Q=\Gamma$ и $x, y \in Q, \gamma \in \Gamma$. Положим $\gamma(x, y)=\gamma \cdot y$, где ( · ) - произведение моноида Г. Условие

$$
\gamma_{1} \cdot \gamma_{2}(x, y)=\gamma_{1}\left(x, \gamma_{2}(x, y)\right)
$$

здесь следует из ассоциативности умножения в $\Gamma$. Кроме того, $e(x, y)=e \cdot y=y$. Наличие единищы $e \in$ Г обеспечивает точность бинарного представления:

$$
\gamma_{1} \neq \gamma_{2} \rightarrow \gamma_{1}(x, e) \neq \gamma_{2}(x, e)
$$

Вьполнимость указанных сверхтождеств очевидна.

Допустим теперь, что Г есть полугруппа без единицы. Мы можем присоединить к Г внешним образом единищу и прийти к моноиду $\Gamma^{*}$. Точное бинарное представление моноида $\Gamma^{*}$ индуцирует точное бинарное представление полугруппы $Г$. Возникают следуюшие утверждения.

ПРЕДЛОЖЕНИЕ 1.4 (бинарная теорема Кэли для полугрупп). Каждая полугруnпа имеет точное бинарное представление, удовлетворяющее сверхтождест-

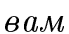

$$
\begin{aligned}
X(x, X(y, z)) & =X(X(x, y), X(x, z)), \\
X(X(x, y), X(u, v)) & =X(X(x, u), X(y, v)) .
\end{aligned}
$$

ПРЕДЛОЖЕНИЕ 1.5 (бинарная теорема Кэли для идемпотентных полугрупп). Каждая идемпотентная полугруппа имеет точное бинарное представление, удовлетворяющее сверхтождествам

$$
\begin{aligned}
X(x, X(y, z)) & =X(X(x, y), z) \\
X(x, X(y, z)) & =X(X(x, y), X(x, z)), \\
X(X(x, y), X(u, v)) & =X(X(x, u), X(y, v)) .
\end{aligned}
$$


ПРЕДЛОЖЕНИЕ 1.6 (бинарная теорема Кэли для коммутативных полугрупп). Каждая коммутативная полугруппа имеет точное бинарное представление, удовлетворяющее сверхтождествам левой дистрибутивности $\left(d_{1}\right)$ и абелевости (1.1). И обратно, если полугруппа имеет такое точное бинарное представление, тогда она коммутативна.

ДоказАТельство. Необходимость очевидна. Достаточность:

$$
\begin{aligned}
\alpha \cdot \beta(x, y) & =\alpha(x, \beta(x, y))=\beta(\alpha(x, x), \alpha(x, y)) \\
& =\alpha(\beta(x, x), \beta(x, y))=\beta(x, \alpha(x, y))=\beta \cdot \alpha(x, y) .
\end{aligned}
$$

Бинарное представление

$$
\varphi: \Gamma \rightarrow \mathscr{F}_{Q}^{2}
$$

называется транзитивным, если множество $Q$ одноэлементно или для любых $a, b, c \in$ $Q$, где $b \neq a \neq c$, сушествует такой элемент $\alpha \in \Gamma$, что $\alpha(a, b)=c$.

Теорема 1.1 (бинарная теорема Кэли для групп). Моноид является группой тогда и только тогда, когда он имеет точное транзитивное и обратимое справа бинарное представление, удовлетворяющее сверхтождеству левой дистрибутивности $\left(d_{1}\right)$.

ДокаЗАТЕЛЬство. Достаточность. Пусть $\Gamma-$ моноид и пусть $\varphi: \Gamma \rightarrow \mathscr{F}_{Q}^{2}-$ точное, транзитивное и обратимое справа бинарное представление $\Gamma$, удовлетворяющее сверхтождеству $\left(d_{1}\right)$. Утверждение в случае $|Q|=1$ - тривиально. Пусть $|Q|=2$. Тогда сушествуют всего четыре обратимые справа бинарные операции, определенные на этом множестве $Q$, которые в совокупности образуют четверную группу Клейна относительно правого умножения операций. Эта группа не имеет подполугрупп, отличных от подгрупш. Поэтому, если моноид Г вкладьвается в эту группу, то и он является группой. Остается рассмотреть случай $|Q| \geqslant 3$.

Из условия обратимости справа следует, что отображение $\alpha_{a}(x)=\alpha(a, x)$ является биекцией множества $Q$, а из сверхтождества $\left(d_{1}\right)$ следует, что $\alpha_{a}$ - автоморфизм бинарного Г-полигона $Q$. Если два автоморфизма совпадают на некотором элементе $b \in Q$, то они будут совпадать и на подполигоне Г-полигона $Q$, порожденном этим элементом $b \in Q$. Согласно определению транзитивности представления бинарньй $\Gamma$-полигон $Q$ порождается любьми двумя его неравными элементами, поэтому если автоморфизмы $\alpha, \beta$ совпадают на двух различных элементах из $Q$, тогда они будут совпадать и на любом элементе $x \in Q$.

Далее, докажем ипликацию:

$$
\alpha(a, b)=\beta(a, b) \rightarrow \alpha=\beta
$$

где $a, b \in Q, a \neq b, \alpha, \beta \in \Gamma$. Рассмотрим две возможности.

1) Бинарньй $Г$-полигон $Q$ идемпотентен, т.е. $\alpha(x, x)=x$ для любых $\alpha \in \Gamma, x \in Q$. В этом случае $\alpha(a, a)=\beta(a, a)$ и автоморфизмы $\alpha_{a}$ и $\beta_{a}$ совпадают на двух различных элементах $a \neq b$. Следовательно, $\alpha_{a}(x)=\beta_{a}(x)$ для любого $x \in Q$, т.е. $\alpha(a, x)=\beta(a, x)$. Однако по условию $|Q| \geqslant 3$, и по транзивности для любого $y \in Q$ 
сушествуют $c \in Q, y \neq c \neq a$, и $\delta \in \Gamma$ со свойством $y=\delta(c, a)=\delta_{c}(a)$, и поскольку $\delta_{c}$ - автоморфизм бинарного Г-полигона $Q$, то

$$
\begin{aligned}
\delta_{c}(\alpha(a, x)) & =\delta_{c}(\beta(a, x)), \\
\alpha\left(\delta_{c}(a), \delta_{c}(x)\right) & =\beta\left(\delta_{c}(a), \delta_{c}(x)\right), \\
\alpha(y, u) & =\beta(y, u)
\end{aligned}
$$

для любых $y, u \in Q$.

2) Бинарньй $\Gamma$-полигон $Q$ не является идемпотентным, т.е. существуют $\gamma \in \Gamma$ и $x \in Q$ такие, что $\gamma(x, x) \neq x$. Тогда $\gamma(y, y) \neq y$ для любого $y \in Q$.

Докажем это. Пусть $\gamma(t, t)=t$ для некоторого $t \in Q, t \neq x$. Опять по условию $|Q| \geqslant 3$ и по транзитивности сушествуют $c \in Q, x \neq c \neq t$, и $\delta \in \Gamma$ со свойством $x=\delta(c, t)=\delta_{c}(t)$, поэтому

$$
\begin{aligned}
\delta_{c}(\gamma(t, t)) & =\delta_{c}(t), \\
\gamma\left(\delta_{c}(t), \delta_{c}(t)\right) & =\delta_{c}(t), \\
\gamma(x, x) & =x,
\end{aligned}
$$

что противоречит выбору элементов $\gamma \in \Gamma, x \in Q$.

Следовательно, подполигон, порожденньй элементом $b \in Q$, содержит по крайней мере два различных элемента $b \neq \gamma(b, b)$ (и, следовательно, совпадает с $Q$ ). Таким образом, автоморфизмы $\alpha_{a}$ и $\beta_{a}$ будут совпадать на любом $x \in Q$, т.е. $\alpha(a, x)=\beta(a, x)$, а отсюда, как и в случае 1$)$, выводится равенство $\alpha=\beta$.

Рассмотрим подг руппу $(\Gamma)$ группы $\mathscr{F}_{Q}^{r}(\cdot)$, порожденную $\Gamma \subseteq \mathscr{F}_{Q}^{r}$. Согласно следствию 1.1 в $(\Gamma)$-алгебре $Q$ также вьполняется сверхтождество $\left(d_{1}\right)$. Следовательно, существует транзитивное, точное и обратимое справа бинарное представление $\psi:(\Gamma) \rightarrow \mathscr{F}_{Q}^{2}$, удовлетворяюшее сверх тождеству левой дистрибутивности $\left(d_{1}\right)$ и продолжаюшее представление $\varphi$. Для завершения доказательства достаточности покажем равенство $(\Gamma)=\Gamma$.

Пусть $\alpha \in(\Gamma)$. Существуют элементы $b \neq a \neq c$ такие, что $\alpha(a, b)=c$. В самом деле, фиксируя $a \in Q$ и выбирая элемент $b$ со свойством $b \neq a, b \neq \alpha^{-1}(a, a)$ (возможность такого выбора обеспечивается условием $|Q| \geqslant 3)$, получим $\alpha(a, b)=c \neq a$. Действительно, если бы $\alpha(a, b)=a$, тогда

$$
\alpha^{-1}(a, \alpha(a, b))=\alpha^{-1}(a, a), \quad \alpha^{-1} \cdot \alpha(a, b)=\alpha^{-1}(a, a)
$$

и $b=\alpha^{-1}(a, a)$, что противоречит выбору элемента $b$. В силу транзитивности исходного бинарного представления $\varphi$ существует такой $\beta \in \Gamma$, что $\beta(a, b)=c$. Следовательно, $\alpha(a, b)=\beta(a, b)$, где $a \neq b$, и потому $\alpha=\beta \in \Gamma$.

Необходимость. Пусть $\Gamma$ - группа. Каждому элементу $\gamma \in \Gamma$ сопоставим бинарную операцию, определенную на множестве $Q=\Gamma: \gamma(x, y)=y x^{-1} \gamma^{-1} x$. Тогда:

$$
\begin{gathered}
e(x, y)=y, \\
\gamma_{1} \cdot \gamma_{2}(x, y)=y x^{-1}\left(\gamma_{1} \gamma_{2}\right)^{-1} x=y x^{-1} \gamma_{2}^{-1} \gamma_{1}^{-1} x \\
=y x^{-1} \gamma_{2}^{-1} x x^{-1} \gamma_{1}^{-1} x=\gamma_{2}\left(x, y x^{-1} \gamma_{2}^{-1} x\right)=\gamma_{1}\left(x, \gamma_{2}(x, y)\right),
\end{gathered}
$$


т.е. имеем бинарное представление группы Г. Это бинарное представление является точным, транзитивньм и обратимым справа. Непосредственно проверяется также, что построенное бинарное представление группы $Г$ удовлетворяет сверхтождеству левой дистрибутивности $\left(d_{1}\right)$ :

$$
\begin{aligned}
\gamma_{2}\left(\gamma_{1}(x, y), \gamma_{1}(x, z)\right) & =z x^{-1} \gamma_{1}^{-1} x\left(y x^{-1} \gamma_{1}^{-1} x\right)^{-1} \gamma_{2}^{-1} y x^{-1} \gamma_{1}^{-1} x \\
& =z x^{-1} \gamma_{1}^{-1} x x^{-1} \gamma_{1} x y^{-1} \gamma_{2}^{-1} y x^{-1} \gamma_{1}^{-1} x \\
& =z y^{-1} \gamma_{2}^{-1} y x^{-1} \gamma_{1}^{-1} x=\gamma_{1}\left(x, \gamma_{2}(y, z)\right)
\end{aligned}
$$

Следующий результат показьвает, что на язьке бинарных представлений класс мультипликативных групп полей выделяется в классе всех групп одним сверхтождеством идемпотентности (ср. с теоремой 1.1$){ }^{3}$

ТЕОРема 1.2 ([18], бинарная теорема Кэли для мультипликативных групп полей). Моноид является мультипликативной группой некоторого поля тогда и только тогда, когда он имеет точное, транзитивное и обратимое справа бинарное представление, удовлетворяющее сверхтождествам левой дистрибутивности $\left(d_{1}\right)$ и идемпотентности $X(x, x)=x$.

ДокаЗАТЕльство. Достаточность. Пусть моноид Г имеет точное, транзитивное и обратимое справа бинарное представление $\varphi: \Gamma \rightarrow \mathscr{F}_{Q}^{2}$, удовлетворяющее сверхтождествам левой дистрибутивности $\left(d_{1}\right)$ и идемпотентности. Согласно теореме 1.1 моноид Г является группй. Из сверхтождества идемпотентности вытекает, что эта группа абелева. Действительно:

$$
\alpha \cdot \beta(x, y)=\alpha(x, \beta(x, y))=\alpha(\beta(x, x), \beta(x, y))=\beta(x, \alpha(x, y))=\beta \cdot \alpha(x, y) .
$$

При условии $|Q| \leqslant 2$ утверждение очевидно, так как единственная идемпотентная и обратимая справа бинарная операция, определенная на таком множестве $Q$, - операция $E(x, y)=y$, поэтому будем предполагать $|Q| \geqslant 3$. Здесь можно предположить также, что группа Г нетривиальна.

Из транзитивности представления $\varphi$ следует, что группа Aut $Q$ всех автоморфизмов бинарного Г-полигона $Q$ является минимальной дважды транзитивной в смысле [26], т.е. для любых двух пар $(a, b)$ и $(c, d)$ элементов из $Q$ с условием $a \neq b, c \neq d$ сушествует единственньй автоморфизм $\lambda \in \operatorname{Aut} Q$ такой, что $\lambda a=c, \lambda b=d$. Для доказательства рассмотрим следующие возможности.

1) $c \neq b$. Тогда существует $\alpha \in \Gamma$ со свойством $\alpha(b, a)=c, \alpha(b, b)=b$, т.е. $\alpha_{b}(a)=c$ и $\alpha_{b}(b)=b$. Сушествует также $\beta \in \Gamma$ со свойством $\beta(c, b)=d, \beta(c, c)=c$, т.е. $\beta_{c}(b)=d$ и $\beta_{c}(c)=c$. Поэтому произведение $\lambda=\alpha_{b} \cdot \beta_{c}$ - единственный автоморфизм с требуемьм свойством.

2) $d \neq a$. Рассмотрение аналогично случаю 1$)$.

3) $c=b, d=a$. В этом случае согласно предположению $|Q| \geqslant 3$ выбираем элемент $s \in Q$, где $s \neq a, b$, и применяем случай 1$)$ к парам $(a, b),(s, a)$ и $(s, a),(b, a)$. Произведение полученных двух автоморфизмов будет искомым автоморфизмом.

\footnotetext{
${ }^{3}$ Хорошо известно, что класс мультипликативных групп полей не может быть охарактеризован (аксиоматизирован) элементарно (А. И. Мальцев, С. Р. Когаловский, G. Sabbagh).
} 
Пусть $\alpha \in \Gamma \backslash\{e\}$ и $a, b \in Q, a \neq b$. Тогда $\alpha(a, b) \neq b$ и, следовательно, существует $f(\alpha) \in \Gamma$ со свойством $f(\alpha)(b, a)=\alpha(a, b)$. Если $x, y \in Q, x \neq y$, тогда согласно дважды транзитивности групшы Aut $Q$ существует автоморфизм $\lambda \in \operatorname{Aut} Q$ со свойством $\lambda a=x, \lambda b=y$. Поэтому

$$
\begin{aligned}
\lambda(f(\alpha)(b, a)) & =\lambda(\alpha(a, b)), \\
f(\alpha)(\lambda b, \lambda a) & =\alpha(\lambda a, \lambda b), \\
f(\alpha)(y, x) & =\alpha(x, y) .
\end{aligned}
$$

Однако последнее равенство, очевидно, выполняется и при $x=y$. Кроме того, если к группе Г внешним образом присоединим нуль 0 , определяя ее бинарное действие по правилу $0(x, y)=x$, a $f(0)=e$, тогда полученное тождество будет справедливым и в случае $\alpha=0$. Справедливо также равенство

$$
f(f(\alpha) \cdot f(\beta))(x, y)=\left(\beta \cdot f\left(\alpha \cdot f\left(\beta^{-1}\right)\right)\right)(x, y)
$$

для любых $\alpha \in \Gamma \cup\{0\}, \beta \in \Gamma, x, y \in Q$. Действительно:

$$
\begin{aligned}
& f(f(\alpha) f(\beta))(x, y)=( \\
& =f(\alpha) \cdot f(\beta))(y, x) \\
& \begin{aligned}
\left(\beta \cdot f\left(\alpha \cdot f\left(\beta^{-1}\right)\right)\right)(x, y) & =\beta\left(x, f\left(\alpha \cdot f\left(\beta^{-1}\right)\right)(x, y)\right) \\
& =\beta\left(x,\left(\alpha \cdot f\left(\beta^{-1}\right)\right)(y, x)\right)=\beta\left(x, \alpha\left(y, f\left(\beta^{-1}\right)(y, x)\right)\right) \\
& =\beta\left(x, \alpha\left(y, \beta^{-1}(x, y)\right)\right)=\alpha\left(\beta(x, y), \beta\left(x, \beta^{-1}(x, y)\right)\right) \\
& =\alpha\left(\beta(x, y),\left(\beta \cdot \beta^{-1}\right)(x, y)\right)=\alpha(\beta(x, y), e(x, y)) \\
& =\alpha(\beta(x, y), y) .
\end{aligned}
\end{aligned}
$$

После этого доказательство достаточности можно завершить с использованием следующего утверждения [27]: коммутативная група $\Gamma(\cdot)$ с единицей $e \in \Gamma$ является мультипликативной групой некоторого поля тогда и только тогда, когда сушествует отображение $f: \Gamma \cup\{0\} \rightarrow \Gamma \cup\{0\}$ со свойствами $f(0)=e, f(f(\alpha))=\alpha$ и

$$
f(f(\alpha) \cdot f(\beta))=\beta \cdot f\left(\alpha \cdot f\left(\beta^{-1}\right)\right)
$$

для любых $\alpha \in \Gamma \cup\{0\}, \beta \in \Gamma$.

Необходимость. Пусть $\Gamma$ - мультипликативная группа некоторого поля $Q(+, \cdot)$, $\Gamma=Q \backslash\{0\}$. Каждому элементу $\gamma \in \Gamma$ сопоставим бинарную операцию, определенную на множестве $Q$ :

Тогда $1(x, y)=y$ и

$$
\gamma(x, y)=(1-\gamma) x+\gamma y \text {. }
$$

$$
\begin{aligned}
\gamma_{1} \cdot \gamma_{2}(x, y) & =\left(1-\gamma_{1} \gamma_{2}\right) x+\gamma_{1} \gamma_{2} y=x-\gamma_{1} \gamma_{2} x+\gamma_{1} \gamma_{2} y \\
& =x-\gamma_{1} x+\gamma_{1} x-\gamma_{1} \gamma_{2} x+\gamma_{1} \gamma_{2} y=x-\gamma_{1} x+\gamma_{1}\left(x-\gamma_{2} x+\gamma_{2} y\right) \\
& =\left(1-\gamma_{1}\right) x+\gamma_{1}\left(\left(1-\gamma_{2}\right) x+\gamma_{2} y\right)=\gamma_{1}\left(x, \gamma_{2}(x, y)\right) .
\end{aligned}
$$

Точность, транзитивность, обратимость справа построенного бинарного представления и справедливость для него сверхтождеств $\left(d_{1}\right)$ и идемпотентности также легко проверяются непосредственно.

Теорема 1.2 доказана.

Общую характеристику минимальных дважды транзитивных групп подстановок см. в $[28]$. 


\section{3. Стохастические алгебры. Понятие котождества}

Пусть $P$ - линейно-упорядоченное поле. Интервал $(0,1) \subseteq P$ - полугрупша относительно умножения поля $P$. Любое бинарное представление этой полугруппы $\Gamma=(0,1)$ в множестве $Q$ можно трактовать как бинарную $\Gamma$-алгебру $Q$ с тождеством

S0. $\quad \alpha \cdot \beta(x, y)=\alpha(x, \beta(x, y))$ для любых $\alpha, \beta \in(0,1)$.

Бинарное представление полугрупш $(0,1)$ в множестве $Q$ называется стохастической алгеброй над $P$ (по другой терминологии - конвексором [29] или барицентрической алгеброй [30]), если оно удовлетворяет следуюшим равенствам:

S1. $\alpha(x, x)=x$,

S2. $\alpha(x, y)=(1-\alpha)(y, x)$,

S3. $\alpha(x, \beta(y, z))=\alpha \beta\left(\frac{\alpha(1-\beta)}{1-\alpha \beta}(x, y), z\right)$

для любых $x, y, z \in Q$ и $\alpha, \beta \in(0,1)$. Первая аксиома - сверхтождество идемпотентности, вторая и третья $-\forall \exists(\forall)$-тождества коммутативности и ассоциативности.

ПримеР 1.5. В любой стохастической алгебре вьполняются сверхтождества левой и правой дистрибутивности $\left(d_{1}\right),\left(d_{2}\right)$ :

$$
\begin{aligned}
\beta(\alpha(x, y), \alpha(x, z)) & \stackrel{S 3 .}{=} \beta \alpha\left(\frac{\beta(1-\alpha)}{1-\beta \alpha}(\alpha(x, y), x), z\right) \\
& \stackrel{S 2 .}{=} \beta \alpha\left(\frac{1-\beta \alpha-\beta+\beta \alpha}{1-\beta \alpha}(x, \alpha(x, y)), z\right) \\
& \stackrel{S 0 .}{=} \beta \alpha\left(\frac{(1-\beta) \alpha}{1-\beta \alpha}(x, y), z\right) \stackrel{S 3 .}{=} \alpha(x, \beta(y, z)) .
\end{aligned}
$$

Именно с учетом этого факта получена характеризация решеток идеалов стохастических алгебр [9] (задача Л. А. Скорнякова): решетка идеалов каждой стохастической алгебры изоморфна решетке идеалов некоторой полурешетки. Каждый идеал стохастической алгебры является пересечением простых идеалов.

В частности, решетка идеалов конечно-порожденной стохастической алгебры - конечна.

Элемент $x$ линейно-упорядоченного поля $P$ называется конечным, если модуль $|x|<n$ для некоторого $n$. Конечные элементы поля $P$ образуют подкольцо $F$. Поскольку $(0,1) \subseteq F$, то всякое выпуклое подмножество $Q \quad F$-модуля естественно преврашается в стохастическую алгебру, если положить $\alpha(x, y)=(1-\alpha) x+\alpha y$, где $\alpha \in(0,1), x, y \in Q$. Такие стохастические алгебры называются аффинными. Пример не аффинной стохастической алгебры можно получить из любой полурешетки $Q(\cdot)$, если положить $\alpha(x, y)=x \cdot y$ для любого $\alpha \in(0,1)$. Такие стохастические алгебры выделяются в классе всех стохастических алгебр сверхтождеством $X(x, y)=Y(x, y)$. Каждая обратимая (слева) стохастическая алгебра будет аффинной [31].

В заключение параграфа определим понятие котождества [8] как абсолютно-замкнутую формулу 2-го порядка с ограниченными функциональными кванторами:

$$
\exists x_{1}, \ldots, x_{n} \forall X_{1}, \ldots, X_{m}\left(w_{1}=w_{2}\right) .
$$


Котождество (1.4) называется $T$-котождеством, если оно - $T$-формула, т.е. $\left\{\left|X_{1}\right|, \ldots,\left|X_{m}\right|\right\} \subseteq T$.

Будем говорить, что в $T$-алгебре $\mathscr{A}=(Q ; \Sigma)$ вьполняется $T$-котождество (1.4), если существуют значения предметных переменных $x_{1}, \ldots, x_{n}$ из $Q$ такие, что равенство $w_{1}=w_{2}$ вьполняется, когда в нем каждая функциональная переменная заменяется любой операцией из $\Sigma$ соответствуюшей арности.

Котождество (1.4) обычно записьвается без кванторной приставки, с заменой в равенстве $w_{1}=w_{2}$ предметных переменных соответствуюшими фиксированньми значениями из $Q$.

ПримеР 1.6. В любой мультиоператорной $\Omega$-групе [32] вьполняется котождест$\mathrm{BO}$

$$
X(0, \ldots, 0)=0,
$$

т.е. 0 - идемпотентный элемент рассматриваемой алгебры, где 0 - единичньй элемент $\Omega$-групшы.

ПримеР 1.7 [31]. Пусть $P$-поле действительных чисел, $(0,1) \subseteq P$ и $\mathscr{A}=\langle Q ;(0,1)\rangle$ - стохастическая алгебра над $P$. Если в алгебре $\mathscr{A}$ вьполняется соотношение $\lambda(a, c)=$ $\lambda(b, c)$, то в ней вьполняется и котождество $X(a, c)=X(b, c)$. Если в алгебре $\mathscr{A}$ выполняется соотношение $\lambda(a, b)=\mu(a, b)$, где $\lambda \neq \mu$, то в ней вьполняется и котождество $X(a, b)=Y(a, b)$.

Пример 1.8 (Дж. фон Нейман). Пусть $L(+, \cdot)$ - модулярная решетка и $a, b, c \in L$. Подрешетка в $L$, порожденная элементами $a, b, c$, будет дистрибутивной тогда и только тогда, когда в $L(+, \cdot)$ вьполняется котождество дистрибутивности

$$
X(a, Y(b, c))=Y(X(a, b), X(a, c)) .
$$

\section{§. Категория арифметически однотипных алгебр}

\section{1. Гомоморфизмы и автоморфизмы арифметически однотипных алгебр}

Пусть $\mathscr{A}=(Q ; \Sigma)$ и $\mathscr{A}^{\prime}=\left(Q^{\prime} ; \Sigma^{\prime}\right)-$ арифметически однотипные алгебры ${ }^{4}$ или $T_{\mathscr{A}} \subseteq T_{\mathscr{A}^{\prime}} ;$ пару $(\varphi, \widetilde{\psi})$ отображений $\varphi: Q \rightarrow Q^{\prime}, \widetilde{\psi}: \Sigma \rightarrow \Sigma^{\prime}$ назовем гомоморфизмом [8] из алгебры $\mathscr{A}$ в алгебру $\mathscr{A}^{\prime}$ и обозначим $(\varphi, \widetilde{\psi}): \mathscr{A} \Rightarrow \mathscr{A}^{\prime}$, если отображение $\widetilde{\psi}$ сохраняет арность операций и для любой операции $A \in \Sigma,|A|=n$, справедливо равенство

$$
\varphi A\left(x_{1}, \ldots, x_{n}\right)=[\widetilde{\psi}(A)]\left(\varphi x_{1}, \ldots, \varphi x_{n}\right)
$$

для любых $x_{1}, \ldots, x_{n} \in Q$.

Помимо полулинейных преобразований $(\varphi, \widetilde{\psi})$ линейных пространств, линейных алгебр и модулей [33], [34] введенные морфизмы охватывают концепцию слабого гомоморфизма, восходящую к Марчевскому [35] (см. также [36]-[38]), понятие полиморфизма, введенное Бурбаки [39], и др. ${ }^{5}$

\footnotetext{
${ }^{4}$ Для простоты в работе рассматриваются алгебры без нуларных операций.

${ }^{5}$ Слабые гомоморфизмы (автоморфизмы) изучались в работах многих авторов (K. Denecke, J. Dudek, A. W. Higgins, T. Katrinak, M. Kolibiar, J. Michalski, E. Plonka, L. Polak, D. Schweigert, A. Solecki, S. Swierczkowski, K. Urbanik, ... ).
} 
Для гомоморфизмов $(\varphi, \widetilde{\psi})$ стандартным образом вводятся понятия эпиморфизма, мономорфизма, изоморфизма, автоморфизма и эндоморфизма. Гомоморфизм $(\varphi, \widetilde{\psi})$ называется эпиморфизмом, если $\varphi, \widetilde{\psi}$ сюръективны, и мономорфизмом, если отображения $\varphi, \widetilde{\psi}$ инъективны. Изоморфизм - это одновременно эпи- и мономорфизм. Гомоморфизм (изоморфизм) $(\varphi, \widetilde{\psi}) T$-алгебры в себя назьвается ее эндоморфизмом (автоморфизмом). Множество всех эндоморфизмов (автоморфизмов) $(\varphi, \widetilde{\psi})$ одной и той же $T$-алгебры образует полугрупп с единищей (соответственно групп) относительно покомпонентного умножения пар: $\left(\varphi_{1}, \widetilde{\psi_{1}}\right) \cdot\left(\varphi_{2}, \widetilde{\psi_{2}}\right)=\left(\varphi_{1} \varphi_{2}, \widetilde{\psi_{1}} \widetilde{\psi_{2}}\right)$.

В качестве примера отметим законы де Моргана в булевых алгебрах, определяюшие автоморфизм $(\varphi, \widetilde{\psi})$ булевой алгебры, где $\varphi$ - взятие дополнения, а отображение $\widetilde{\psi}$ отлично от тождественного.

Группа всех автоморфизмов $(\varphi, \widetilde{\psi})$ одной и той же $T$-алгебры $\mathscr{A}$ обозначим через Aut $\mathscr{A}$.

Автоморфизмы вида $(\varphi, \widetilde{\varepsilon})$, где $\widetilde{\varepsilon}-$ тождественное отображение, называются главными или обычными автоморфизмами, их совокупность - группа, обозначаемая $\mathrm{Aut}^{(\circ)} \mathscr{A}$ и, очевидно, являюшаяся инвариантной подгруппой в группе Aut $\mathscr{A}$.

ТЕОРема 2.1. Пусть $G$ - произвольная группа с произвольной инвариантной nодгруппой $H$. Cуществует алгебра $\mathscr{A}$ такая, что $G \simeq$ Aut $\mathscr{A}$ u $H \simeq \operatorname{Aut}^{(\circ)} \mathscr{A}$, причем последний изоморфизм индуцируется первым изоморфизмом.

ДокАЗАТЕльство (ср. [20], а также [19]). ${ }^{6}$ Пусть

$$
Q=(G \times\{0,1\}) \cup\{a, b, c, d\},
$$

где $(G \times\{0,1\}) \cap\{a, b, c, d\}=\varnothing$. Пусть $G \times\{0\}-$ вполне-упорядоченное множество относительно порядка " $\leqslant$ ". По каждому элементу $g \in G$ определим унарную операцию $g^{*}$ на $Q$ следуюшим образом:

$$
\begin{gathered}
g^{*}(h, 0)=(g, 0), \quad \text { если }(g, 0) \leqslant(h, 0), \quad g \neq h, \\
g^{*}(h, 0)=b, \quad \text { если }(g, 0) \geqslant(h, 0), \\
g^{*}(h, 1)=(h g, 1) \quad \text { для любого } h \in G, \\
g^{*}(a)=(g, 0), \quad g^{*}(b)=b, \quad g^{*}(c)=c, \quad g^{*}(d)=d .
\end{gathered}
$$

Аналогично, по каждому $S \in G / H$ определяется унарная операция $S^{*}$ на $Q$ по правилу:

$$
\begin{aligned}
& S^{*}(h, 0)=(h, 0) \quad \text { для любого } h \in G, \\
& S^{*}(h, 1)=c, \quad \text { если } h \in S, \\
& S^{*}(h, 1)=d, \quad \text { если } h \notin S, \\
& S^{*}(a)=S^{*}(b)=b, \quad S^{*}(c)=S^{*}(d)=d .
\end{aligned}
$$

Очевидно, $g_{1}^{*}=g_{2}^{*} \Leftrightarrow g_{1}=g_{2}$ и $S_{1}^{*}=S_{2}^{*} \Leftrightarrow S_{1}=S_{2}$. В результате получаем унарную алгебру $\mathscr{A}=\langle Q ; \Sigma\rangle$, где $\Sigma=\left\{g^{*} \mid g \in G\right\} \cup\left\{S^{*} \mid S \in G / H\right\}$.

\footnotetext{
${ }^{6}$ Предполагается аксиома выбора.
} 
Для любого $k \in G$ определяя отображение $\varphi_{k}: Q \rightarrow Q$ по правилу:

$$
\begin{array}{clll}
\varphi_{k}(h, 1)=(k h, 1) & \text { для любого } & h \in G, \\
\varphi_{k}(z)=z & \text { для любого } & z \in(G \times\{0\}) \cup\{a, b, c, d\},
\end{array}
$$

замечаем, что вьполнены равенства:

$$
\begin{aligned}
\varphi_{k} g^{*}(x) & =g^{*}\left(\varphi_{k} x\right), \\
\varphi_{k} S^{*}(x) & =(k S)^{*}\left(\varphi_{k} x\right)
\end{aligned}
$$

для любых $k, g \in G, S \in G / H$, т.е. $\left(\varphi_{k}, \widetilde{\psi}_{k}\right) \in \operatorname{Aut} \mathscr{A}$, где $\widetilde{\psi}_{k}\left(g^{*}\right)=g^{*}$ и $\widetilde{\psi}_{k}\left(S^{*}\right)=$ $(k S)^{*}$. При этом

$$
\left(\varphi_{k}, \widetilde{\psi}_{k}\right) \in \operatorname{Aut}^{(\circ)} \mathscr{A} \Leftrightarrow \widetilde{\psi}_{k}=\widetilde{\varepsilon} \Leftrightarrow(k S)^{*}=S^{*} \Leftrightarrow k S=S \Leftrightarrow k \in H \unlhd G .
$$

Соответствие $k \rightarrow\left(\varphi_{k}, \widetilde{\psi}_{k}\right)$ является мономорфизмом групп $G \rightarrow$ Aut $\mathscr{A}$. Остается доказать,что алгебра $\mathscr{A}$ не имеет других автоморфизмов $(\varphi, \widetilde{\psi})$ кроме автоморфизмов вида $\left(\varphi, \widetilde{\psi}_{k}\right), k \in G$. Пусть $(\varphi, \widetilde{\psi}) \in$ Aut $\mathscr{A}$.

Множество $Q$ представим как дизъюнктную сумму двух подмножеств: $Q_{0}=$ $(G \times\{0\}) \cup\{a, b\}, Q_{1}=(G \times\{1\}) \cup\{c, d\}$, каждое из которых содержит по одному одноэлементному подмножеству, замкнутому относительно всех операций из $\Sigma$. Для подмножества $Q_{0}$ это $\{b\}$, а для $Q_{1}$ - это $\{d\}$. Поскольку элемент $a-$ единственный элемент $Q$, не являюшийся значением какой-либо операции из $\Sigma$, то $\varphi \underset{a}{a}=a$. Действительно, если $\varphi a=y \neq a$, то сушествуют $A \in \Sigma, x \in Q$ такие, что $(\widetilde{\psi} A)(\varphi x)=y$, поэтому

$$
\varphi A(x)=(\widetilde{\psi} A)(\varphi x)=y,
$$

где $A(x) \neq a$, что противоречит инъективности $\varphi$.

Для любого $g \in G$ сушествует операция $X \in \Sigma$ такая, что

$$
\begin{gathered}
\varphi g^{*}(x)=X(\varphi x), \\
X(x)=\varphi\left(g^{*}\left(\varphi^{-1} x\right)\right)
\end{gathered}
$$

для любого $x \in Q$. Поэтому

$$
\varphi(g, 0)=\varphi\left(g^{*}(a)\right)=\varphi\left(g^{*}\left(\varphi^{-1} a\right)\right)=X(a) .
$$

Если $X=S^{*}$ для некоторого $S \in G / H$, то $X(a)=b$ и $\varphi(g, 0)=b$, т.е. $\varphi^{-1} b=$ $(g, 0)$, что невозможно, поскольку $\{b\}$ замкнуто относительно всех операций из $\Sigma$, однако $\{(g, 0)\}$ не является таковым. Следовательно, $X=h^{*}$ для некоторого $h \in G$ и $X(a)=h^{*}(a)=(h, 0)$, т.е. $\varphi(g, 0)=(h, 0)$. Аналогично доказывается, что для любого элемента $(g, 0) \in G \times\{0\}$ сушествует элемент $(h, 0) \in G \times\{0\}$ такой, что $\varphi(h, 0)=(g, 0)$. Итак, $\varphi(G \times\{0\})=G \times\{0\}$. 
Согласно определению операций из $\Sigma$ имеем $(g, 0) \leqslant(h, 0)$ тогда и только тогда, когда сушествует операция $X \in \Sigma$ такая, что $X(h, 0)=(g, 0)$. Если $X \in \Sigma$, то и операция $X^{\prime}(x)=\varphi\left(X\left(\varphi^{-1}(x)\right)\right)$ содержится в $\Sigma$, поэтому

$$
\begin{gathered}
(g, 0)=X(h, 0)=\varphi^{-1}\left(X^{\prime}(\varphi(x, 0))\right) \\
\varphi(g, 0)=X^{\prime}(\varphi(h, 0))
\end{gathered}
$$

т.е. $\varphi(g, 0) \leqslant \varphi(h, 0)$, и отображение $\varphi$ сохраняет порядок " $\leqslant$ ".

Далее, методом трансфинитной индукции доказьвается равенство $\varphi(g, 0)=(g, 0)$ для любого $(g, 0) \in G \times\{0\}$. Действительно, для наименьшего элемента это очевидно. Пусть для всех элементов $(x, 0) \in G \times\{0\}$, строго меньших $(a, 0) \in G \times\{0\}$, справедливо равенство $\varphi(x, 0)=(x, 0)$. Докажем равенство $\varphi(a, 0)=(a, 0)$. Возможны три случая. Если $\varphi(a, 0)<(a, 0)$, тогда по индуктивному предположению $\varphi(a, 0)=\varphi^{2}(a, 0)<\varphi(a, 0)$. Если же $(a, 0)<\varphi(a, 0)$, тогда, рассматривая элемент $(h, 0) \in G \times\{0\}$ со свойством $\varphi(h, 0)=(a, 0)<\varphi(a, 0)$, получим $(h, 0)<(a, 0)$. С другой стороны, по индуктивному предположению $(h, 0)=\varphi(h, 0)=(a, 0)$. Полученные противоречия доказьвают равенство $\varphi(a, 0)=(a, 0)$.

Теперь докажем равенство

$$
\varphi g^{*}(x)=g^{*}(\varphi x)
$$

для любых $g \in G, x \in Q$. Для любого $g \in G$ существует операщия $f \in \Sigma$ такая, что

$$
\begin{gathered}
\varphi f(x)=g^{*}(\varphi x), \\
f(x)=\varphi^{-1}\left(g^{*}(\varphi x)\right),
\end{gathered}
$$

где $f=S^{*}$ или $f=h^{*}$ для подходящих $S^{*}, h^{*} \in \Sigma$. Если $f=S^{*}$, тогда $\varphi(g, 0)=$ $(g, 0)=g^{*}(a)$, и приходим к противоречию:

$$
(g, 0)=\varphi^{-1}\left(g^{*}(a)\right)=\varphi^{-1}\left(g^{*}(\varphi a)\right)=S^{*}(a)=b .
$$

Если же $f=h^{*}, h \in G$, тогда

$$
(g, 0)=\varphi^{-1}\left(g^{*}(\varphi a)\right)=h^{*}(a)=(h, 0)
$$

и $h=g$, т.е. $f=g^{*}$.

Поскольку $\varphi Q_{0} \ni \varphi a=a$ и $Q_{0}$ замкнуто относительно всех операций из $\Sigma$, то и $\varphi Q_{0}$ обладает этим свойством, поэтому ввиду определения $g^{*}$ имеем $\varphi Q_{0} \supseteq Q_{0}$. Однако с учетом равенств $\varphi(g, 0)=(g, 0), \varphi a=a$ получим $\varphi b=b, \varphi Q_{0}=Q_{0}$. Поэтому $\varphi Q_{1}=Q_{1}$ и $\varphi(d)=d$.

Определим $\varphi c$. Поскольку $\varphi Q_{1}=Q_{1}$ и $\varphi c \neq \varphi d=d$, то $\varphi c=c$ или $\varphi c=(g, 1)$ для подходящего $g \in G$. Однако второй случай исключается. Действительно, во втором случае, выбирая $S \in G / H$ со свойством $g \in S$, получим:

$$
\varphi^{-1}(c)=\varphi^{-1}\left(H^{*}(g, 1)\right)=\varphi^{-1}\left(H^{*}(\varphi c)\right)=A(c),
$$

где $A \in \Sigma$. Таким образом, $\varphi^{-1}(c) \in\{c, d\}$, что является противоречием. Итак $\varphi c=c$. Следовательно, $\varphi(G \times\{1\})=G \times\{1\}$. 
Наконец, пусть $e$ - единица групшы $G$. Обозначая $\varphi(e, 1)=(k, 1)$, где $k \in G$, получим:

$$
\varphi(h, 1)=\varphi h^{*}(e, 1)=h^{*} \varphi(e, 1)=h^{*}(k, 1)=(k h, 1)=\varphi_{k}(h, 1)
$$

для любого $h \in G$, т.е. $\varphi=\varphi_{k}$. А если $\varphi=\varphi_{k}$, то $(\varphi, \widetilde{\psi})=\left(\varphi_{k}, \widetilde{\psi}_{k}\right)$, поскольку в автоморфизме $(\varphi, \widetilde{\psi})$ отображение $\widetilde{\psi}$ определяется единственным образом по $\varphi$.

Теорема 2.1 доказана.

Арифметически однотипные $T$-алгебры и их гомоморфизмы $(\varphi, \widetilde{\psi})$ в качестве морфизмов, образуют категорию, назьваемую категорией $T$-алгебр, в которой морфизмы между алгебрами меняют не только элементы, но и операции. Следующее утверждение показьвает, что $T$-алгебра слов $U(\mathscr{X})$ - свободный объект этой категории.

ПреДЛОЖЕНИЕ 2.1. Пусть $U(\mathscr{X})=\langle(\mathscr{X}) U ; U\rangle$ есть Т-алгебра слов и $\mathscr{A}=$ $\langle Q ; \Sigma\rangle-$ любая T-алгебра. Если $\widetilde{\psi}: U \rightarrow \Sigma-$ произвольное отображсние, сохраняющее арность операций, то каждое отображсене $\varphi_{0}: \mathscr{X} \rightarrow Q$ можно продолжить до отображения $\varphi:(\mathscr{X}) U \rightarrow Q$ так, что пара $(\varphi, \widetilde{\psi})$ есть гомоморфизм из Т-алгебры слов $U(\mathscr{X})$ в $T$-алгебру $\mathscr{A}$ (причем отображение $\varphi$ определяется единственным образом по $\left.\widetilde{\psi} и \varphi_{0}\right)$.

Действительно, во-первых, определим $\left.\varphi\right|_{\mathscr{X}}=\varphi_{0}$, и, во-вторых, если $\omega-$ функциональная переменная с арностью $m$ и образы слов $v_{1}, \ldots, v_{m}$ при отображении $\varphi$ уже определены, то образом слова $\omega\left(v_{1}, \ldots, v_{m}\right)$ считаем элемент $\widetilde{\psi}(\omega)\left(\varphi v_{1}, \ldots, \varphi v_{m}\right) \in Q$.

Следовательно, в $T$-алгебре $\mathscr{A}=\langle Q ; \Sigma\rangle$ выполняется $T$-сверхтождество $w_{1}=w_{2}$ тогда и только тогда, когда при любом гомоморфизме $(\varphi, \widetilde{\psi}): U(\mathscr{X}) \Rightarrow \mathscr{A}$ из $T$-алгебры слов $U(\mathscr{X})$ в $T$-алгебру $\mathscr{A}$, справедливо равенство $\varphi\left(w_{1}\right)=\varphi\left(w_{2}\right)$.

Пусть $\mathscr{A}=\langle Q ; \Sigma\rangle$ и $\mathscr{A}^{\prime}=\left\langle Q^{\prime} ; \Sigma^{\prime}\right\rangle$ две $T$-алгебры. Обозначим через $\operatorname{Hom}\left(\mathscr{A}^{\prime}, \mathscr{A}\right)$ множество всех гомоморфизмов $(\varphi, \widetilde{\psi})$ из $\mathscr{A}^{\prime}$ в $\mathscr{A}$. Отображение $\widetilde{\psi}: \Sigma^{\prime} \longrightarrow \Sigma$ назовем допустимым, если существует отображение $\varphi: Q^{\prime} \rightarrow Q$ такое, что $(\varphi, \widetilde{\psi}) \in$ $\operatorname{Hom}\left(\mathscr{A}^{\prime}, \mathscr{A}\right)$. Для допустимого отображения $\widetilde{\psi}$ множество $\operatorname{Hom}\left(\mathscr{A}^{\prime}, \mathscr{A}\right)$ называется $\widetilde{\psi}$-суммируемым, если для любого $A \in \Sigma,|A|=n$, и для любых гомоморфизмов $\left(\varphi_{1}, \widetilde{\psi}\right), \ldots,\left(\varphi_{n}, \widetilde{\psi}\right) \in \operatorname{Hom}\left(\mathscr{A}^{\prime}, \mathscr{A}\right)$ пара $\left(\lambda_{A}, \widetilde{\psi}\right)$ содержится в $\operatorname{Hom}\left(\mathscr{A}^{\prime}, \mathscr{A}\right)$, где $\lambda_{A}: Q^{\prime} \rightarrow Q$ определяется по правилу:

$$
\lambda_{A}(x)=A\left(\varphi_{1} x, \ldots, \varphi_{n} x\right), \quad x \in Q^{\prime} .
$$

Непустое множество гомоморфизмов $\operatorname{Hom}\left(\mathscr{A}^{\prime}, \mathscr{A}\right)$ назьвается суммируемым, если оно $\widetilde{\psi}$-суммируемо для любого допустимого $\widetilde{\psi}$. Наконец, $T$-алгебра $\mathscr{A}$ называется абелевой, если $\operatorname{Hom}\left(\mathscr{A}^{\prime}, \mathscr{A}\right)$ суммируемо для любой $T$-алгебры $\mathscr{A}^{\prime}$, как только $\operatorname{Hom}\left(\mathscr{A}^{\prime}, \mathscr{A}\right) \neq \varnothing$.

Следующее утверждение вытекает из предложния 2.1:

Т-алгебра Д будет абелевой тогда и только тогда, когда в ней выполняется сверхтохдество абелевости (точнее $(n, m)$-абелевости)

$$
\begin{aligned}
X\left(Y\left(x_{11}, \ldots, x_{1 n}\right), \ldots, Y\left(x_{m 1},\right.\right. & \left.\left.\ldots, x_{m n}\right)\right) \\
& =Y\left(X\left(x_{11}, \ldots, x_{m 1}\right), \ldots, X\left(x_{1 n}, \ldots, x_{m n}\right)\right)
\end{aligned}
$$

для любых $m, n \in T$. 
Пример 2.1. Любая аффинная стохастическая алгебра - абелева, так как в ней вьполняется сверхтождество $(2,2)$-абелевости (1.1), что устанавливается непосредственной проверкой. Поскольку в свободной стохастической алгебре [29] справедливо квазитождество

$$
\alpha(y, x)=\alpha(z, x) \rightarrow y=z,
$$

то свободная стохастическая алгебра будет аффинной [31] и, следовательно, она также является абелевой алгеброй. Поэтому абелевой является и любая стохастическая алгебра.

\section{2. Сверхмногообразия алгебр}

Пусть $\mathscr{L}$ - некоторьй (непустой) набор $T$-сверхтождеств, а $\mathscr{M}_{\mathscr{L}}^{T}-$ класс всех $T$-алгебр, в которых выполняется каждое сверхтождество из $\mathscr{L}$.

Класс $T$-алгебр $\mathscr{N}$ назьвается сверхмногообразием ( $T$-алгебр), если существует система $T$-сверхтождеств $\mathscr{L}$ со свойством

$$
\mathscr{N}=\mathscr{M}_{\mathscr{L}}^{T}
$$

При этом $\mathscr{L}$ называется определяюшей системой сверхтождеств для $\mathscr{N}$.

Например, многообразие полугрупш является сверхмногообразием $T$-алгебр, где $T=\{2\}$, а $\mathscr{L}-$ множество из двух сверхтождеств:

$$
\begin{aligned}
& X(x, X(y, z))=X(X(x, y), z), \\
& X(x, y)=Y(x, y) .
\end{aligned}
$$

Класс всех абелевых $T$-алгебр является сверхмногообразием $T$-алгебр, определяемым системой сверхтождеств $(n \times m)$-абелевости для $n, m \in T$.

Системы $T$-сверхтождеств $\mathscr{L}_{1}, \mathscr{L}_{2}$ назьваются эквивалентными, если

$$
\mathscr{M}_{\mathscr{L}_{1}}^{T}=\mathscr{M}_{\mathscr{L}_{2}}^{T}
$$

По определению система $T$-сверхтождеств $\mathscr{L}$ имеет конечньй базис (или конечно-базируема), если она эквивалентна конечной системе $T$-сверхтождеств $\mathscr{L}_{0}$. Каждому классу $T$-алгебр $\mathscr{M}$ соответствует класс $Л_{\mathscr{M}}^{T}$ всех таких $T$-сверхтождеств, каждое из которых вьполняется во всех алгебрах из $\mathscr{M}$. Говорят, что класс $T$-алгебр $\mathscr{M}$ имеет конечньй базис сверхтождеств, если $\Omega_{\mathscr{M}}^{T}$ конечно-базируема. Пара отображений

$$
\mathscr{L} \rightarrow \mathscr{M}_{\mathscr{L}}^{T}
$$

и

$$
\mathscr{M} \rightarrow \pi_{\mathscr{M}}^{T}
$$

образует соответствие Галуа.

Пересечение сверхмногообразий $T$-алгебр есть сверхмногообразие $T$-алгебр, а именно:

$$
\bigcap_{i \in I} \mathscr{M}_{\mathscr{L}_{i}}^{T}=\mathscr{M}_{\bigcup_{i \in I}^{T}}^{T} \mathscr{L}_{i}
$$


поэтому для любого класса $T$-алгебр $\mathscr{M}$ сушествует наименьшее (относительно теоретико-множественного включения) сверхмногообразие $T$-алгебр $\mathscr{M}^{*} \supset \mathscr{M}$, называемое сверхмногообразием алгебр, порожденным $\mathscr{M}$. Понятно, что $\mathscr{M}^{*}$ определяется системой $T$-сверхтождеств $Л_{\mathscr{M}}^{T}$, поэтому характеризация сверхмногообразия $\mathscr{M}^{*}$ равносильна характеризации всех сверхтождеств класса $T$-алгебр $\mathscr{M}$.

В дальнейшем понятия подалгебры, прямого и подпрямого произведения и др. рассматриваются в смысле категории $T$-алгебр и их гомоморфизмов $(\varphi, \widetilde{\psi})$ (см. [8], [9] и [21]).

Теорема 2.2. Если $T$-алгебра $\mathscr{A}=\langle Q ; \Sigma\rangle$, где $T=\{2\}$, удовлетворяет сверхтождествам

$$
\begin{gathered}
X(x, x)=x, \\
X(x, y)=X(y, x), \\
X(x, X(y, z))=X(X(x, y), z), \\
X(x, Y(y, z))=Y(X(x, y), X(x, z))
\end{gathered}
$$

u подпрямо неразложима, тогда мощность $|\Sigma| \leqslant 2, a|Q| \leqslant 3$.

ДоказАТельство [21]. В случае $|\Sigma| \geqslant 3 T$-алгебра $\mathscr{A}=\langle Q ; \Sigma\rangle$ оказьвается подпрямо разложимой. Действительно, пусть $A_{1}, A_{2}, A_{3}$ - попарно различные бинарные операции из $\Sigma$, и пусть

$$
F_{i, j}(x, y)=A_{i}\left(x, A_{j}(x, y)\right) .
$$

Определим отношения $\Theta_{i, j}$ на множестве $Q$ следующим образом:

$$
x \Theta_{i, j} y \Longleftrightarrow f_{i, j}(x, y)=x, f_{i, j}(y, x)=y,
$$

где $i, j=1,2,3$ и $i<j$. Рефлексивность и симметричность отношения $\Theta_{i, j}$ очевидны, а транзитивность вытекает из сверхтождества

$$
X(x, Y(x, X(y, Y(y, z))))=X(x, Y(x, X(z, Y(z, y)))),
$$

которое является следствием сверхтождеств (2.1)-(2.4). Покажем это. Во-первых, очевидно, что сверхтождество правой дистрибутивности

$$
X(Y(y, z), x)=Y(X(y, x), X(z, x))
$$

является следствием сверхтождеств (2.1)-(2.4). Далее, имеем:

$$
\begin{aligned}
X(x, Y(y, z)) & \stackrel{(2.4)}{=} Y(X(x, y), X(x, z)) \\
& \stackrel{(2.6)}{=} X(Y(x, X(x, z)), Y(y, X(x, z))) \\
& \stackrel{(2.4)}{=} X(X(Y(x, x), Y(x, z)), X(Y(y, x), Y(y, z))) \\
& =X(X(x, Y(x, z)), X(Y(y, x), Y(y, z))),
\end{aligned}
$$


T.e.

$$
X(x, Y(y, z))=X(X(x, Y(x, z)), X(Y(y, x), Y(y, z))) .
$$

Следовательно,

$$
\begin{aligned}
X(x, X(Y(x, y), Y(y, z))) & \stackrel{(2.2)}{=} X(x, X(Y(y, z), Y(x, y))) \\
& \stackrel{(2.3)}{=} X(X(x, Y(y, z)), Y(x, y)) \\
& \stackrel{(2.7)}{=} X(X(X(x, Y(x, z)), X(Y(y, x), Y(y, z))), Y(x, y)) \\
& \stackrel{(2.1)-(2.3)}{=} X(X(x, Y(x, z)), X(Y(y, x), Y(y, z))) \\
& \stackrel{(2.7)}{=} X(x, Y(y, z)),
\end{aligned}
$$

т.e.

$$
X(x, Y(y, z))=X(x, X(Y(x, y), Y(y, z)))
$$

откуда при замене $y$ на $Y(x, y)$ получим сверхтождество

$$
X(x, X(Y(x, y), Y(Y(x, y), z)))=X(x, Y(Y(x, y), z)) .
$$

Далее, заменяя здесь $y$ на $z$ и $z$ на $y$ и применяя сверхтождества (2.2), (2.3), получим сверхтождество:

$$
X(x, X(Y(x, z), Y(Y(x, y), z)))=X(x, Y(Y(x, y), z)) .
$$

Сравнивая сверхтождества $(2.9),(2.10)$, приходим к сверхтождеству

$$
X(x, X(Y(x, y), Y(Y(x, y), z)))=X(x, X(Y(x, z), Y(Y(x, y), z))),
$$

откуда, с использованием сверхтождества (2.4), получим сверхтождество (2.5).

Докажем, что $\Theta_{i, j}=\left(\Theta_{i, j}, \widetilde{O}\right)$ является конгруэнцией алгебры $\mathscr{A}=\langle Q ; \Sigma\rangle$, т.е. если $x \Theta_{i, j} y$ и $u \Theta_{i, j} v$, то $A(x, u) \Theta_{i, j} A(y, v)$ для любой операции $A \in \Sigma$. Достаточно показать, что $A(x, u) \Theta_{i, j} A(y, u)$, где $x \Theta_{i, j} y$ и $x, y, u \in Q$. Воспользуемся сверхтождеством

$$
X(Y(X(x, y), z), Y(y, z))=Y(X(x, y), z)
$$

также являющимся следствием сверхтождеств (2.1)-(2.4):

$$
\begin{aligned}
X(Y(X(x, y), z), Y(y, z)) & \stackrel{(2.6)}{=} X(X(Y(x, z), Y(y, z)), Y(y, z)) \\
& \stackrel{(2.3)}{=} X(Y(x, z), X(Y(y, z), Y(y, z))) \\
& \stackrel{(2.1)}{=} X(Y(x, z), Y(y, z)) \\
& \stackrel{(2.6)}{=} Y(X(x, y), z) .
\end{aligned}
$$


Пусть $x \Theta_{i, j} y$, т.е. $A_{i}\left(x, A_{j}(x, y)\right)=x$ и $A_{i}\left(y, A_{j}(y, x)\right)=y$, где $A_{i}, A_{j} \in \Sigma$. Тогда для любой операции $A \in \Sigma$ имеем:

$$
\begin{aligned}
A(x, u) & =A\left(A_{i}\left(x, A_{j}(x, y)\right), u\right) \\
& \stackrel{(2.11)}{=} A_{i}\left(A\left(A_{i}\left(x, A_{j}(x, y)\right), u\right), A\left(A_{j}(x, y), u\right)\right) \\
& \stackrel{(2.4)}{=} A_{i}\left(A(x, u), A_{j}(A(x, u), A(y, u))\right) .
\end{aligned}
$$

Аналогично получаем равенство:

$$
A(y, u)=A_{i}\left(A(y, u), A_{j}(A(y, u), A(x, u))\right) .
$$

Рассмотрим фактор-алгебру $\mathscr{A} / \Theta_{i, j}$ по конгруэнции $\Theta_{i, j}$. Отображения

$$
\begin{aligned}
& \varphi_{0}: x \rightarrow\left([x]_{1,2},[x]_{1,3},[x]_{2,3}\right), \\
& \widetilde{\psi}_{0}: A \rightarrow\left([A]_{1,2},[A]_{1,3},[A]_{2,3}\right),
\end{aligned}
$$

где $[\alpha]_{i, j}$ - класс эквивалентности элемента $\alpha$ по $\Theta_{i, j}$, определяют мономорфизм алгебры $\mathscr{A}$ в прямое произведение $\mathscr{A} / \Theta_{1,2} \times \mathscr{A} / \Theta_{1,3} \times \mathscr{A} / \Theta_{2,3}$. Гомоморфность этой пары очевидна, а инъективность отображения $\varphi_{0}$, а следовательно, и отображения $\widetilde{\psi}_{0}$, вытекает из следуюшей импликации: если

$$
f_{1,2}(x, y)=f_{1,3}(x, y)=f_{2,3}(x, y)=x,
$$

то

$$
x=A_{1}\left(A_{2}(x, y), A_{3}(x, y)\right) .
$$

Последняя импликация вытекает из следующего сверхтождества (при $X=A_{1}$, $\left.Y=A_{2}, Z=A_{3}\right)$ :

$$
X(Y(x, y), Z(x, y))=X(X(Y(x, Z(x, y)), Y(x, y)), Z(x, y)),
$$

которое вьполняется в алгебре $\mathscr{A}$, поскольку оно также является следствием сверхтождеств (2.1)-(2.4). Действительно, достаточно сверхтождество (2.8) записать в виде

$$
X(Y(x, y), z)=X(X(Y(x, z), Y(x, y)), z)
$$

и вместо $z$ положить $Z(x, y)$.

В результате алгебра $\mathscr{A}$ оказьвается изоморфной подпрямому произведению $\left(\varphi_{0} Q ; \widetilde{\psi}_{0} \Sigma\right)$ алгебр $\mathscr{A} / \Theta_{1,2}, \mathscr{A} / \Theta_{1,3}, \mathscr{A} / \Theta_{2,3}$, проекции которого не являются изоморфизмами. Покажем, например, что первая проекция подпрямого произведения $\left(\varphi_{0} Q ; \widetilde{\psi}_{0} \Sigma\right)$ не является изоморфизмом. Поскольку $A_{1} \neq A_{2}$, то сушествуют $x, y \in Q$ такие, что $A_{1}(x, y) \neq A_{2}(x, y)$, где $x \neq y$. Однако $\left[A_{1}(x, y)\right]_{1,2}=\left[A_{2}(x, y)\right]_{1,2}$, ибо

$$
\begin{aligned}
& f_{1,2}\left(A_{1}(x, y), A_{2}(x, y)\right)=A_{1}(x, y), \\
& f_{1,2}\left(A_{2}(x, y), A_{1}(x, y)\right)=A_{2}(x, y) .
\end{aligned}
$$


Для доказательства последних равенств воспользуемся сверхтождеством

$$
X(X(x, y), Y(X(x, y), Y(x, y)))=X(x, y)
$$

являюшимся следствием сверхтождества (2.8). Действительно, при $y=z$ из сверхтождества (2.8) вытекает сверхтождество

$$
X(x, y)=X(X(x, y), Y(x, y))
$$

Поэтому имеем:

$$
\begin{aligned}
X(x, y) & =X(X(x, y), Y(x, y)) \\
& \stackrel{(2.13)}{=} X(X(X(x, y), Y(x, y)), Y(X(x, y), Y(x, y))) \\
& \stackrel{(2.13)}{=} X(X(x, y), Y(X(x, y), Y(x, y))),
\end{aligned}
$$

т.е. получаем сверхтождество (2.12). Далее:

$$
f_{1,2}\left(A_{1}(x, y), A_{2}(x, y)\right)=A_{1}\left(A_{1}(x, y), A_{2}\left(A_{1}(x, y), A_{2}(x, y)\right)\right) \stackrel{(2.12)}{=} A_{1}(x, y)
$$

Аналогично, с использованием сверхтождества

$$
Y(x, y) \stackrel{(2.1)}{=} X(Y(x, y), Y(x, y)) \stackrel{(2.13)}{=} X(Y(x, y), Y(Y(x, y), X(x, y)))
$$

доказывается второе равенство.

Первая часть теоремы 2.2 доказана. Докажем ее вторую часть (ср. [40], [41]).

Пусть $f \in \Sigma$, и пусть непустое подмножество $I \subseteq Q$ является $f$-замкнутым, т.е.

$$
x, y \in I \longrightarrow f(x, y) \in I,
$$

тогда отношение $\widetilde{f}_{I}$, определенное по правилу

$$
a \widetilde{f}_{I} b \Longleftrightarrow f(a, I) \cap f(b, I) \neq \varnothing,
$$

где $f(a, I)=\{f(a, x) \mid x \in I\}$, будет конгруэнщией для любой алгебры $\mathscr{A}$ со сверхтождествами (2.1)-(2.4). Рефлексивность и симметричность отношения $\widetilde{f}_{I}$ очевидны. Докажем транзитивность.

Пусть $a \widetilde{f}_{I} b$ и $b \widetilde{f}_{I} c$, т.е. сушествуют элементы $x, y, u, v \in I$ такие, что $f(a, x)=$ $f(b, y)$ и $f(b, u)=f(c, v)$. Тогда имеем:

$$
\begin{aligned}
f(a, f(x, u)) & =f(f(a, x), u)=f(f(b, y), u) \\
& =f(b, f(y, u))=f(b, f(u, y))=f(f(b, u), y) \\
& =f(f(c, v), y)=f(c, f(v, y)),
\end{aligned}
$$

где $f(x, u), f(v, y) \in I$, т.е. $a \tilde{f}_{I} c$. 
Пусть $g \in \Sigma$, и пусть $a_{1} \widetilde{f}_{I} a_{2}$ и $b_{1} \widetilde{f}_{I} b_{2}$; докажем условие $g\left(a_{1}, b_{1}\right) \widetilde{f}_{I} g\left(a_{2}, b_{2}\right)$. Существуют $x, y, u, v \in I$ такие, что $f\left(a_{1}, x\right)=f\left(a_{2}, y\right)$ и $f\left(b_{1}, u\right)=f\left(b_{2}, v\right)$. Без ущерба для общности элементы $x, y, u, v$ можно выбрать равными. Действительно, если $\omega=f(x, f(y, f(u, v)))$, тогда $\omega \in I$, и согласно сверхтождествам (2.1)-(2.3) имеем:

$$
\omega=f(x, \omega)=f(y, \omega)=f(u, \omega)=f(v, \omega),
$$

следовательно,

$$
\begin{aligned}
f\left(a_{1}, \omega\right) & =f\left(a_{1}, f(x, \omega)\right)=f\left(f\left(a_{1}, x\right), \omega\right) \\
& =f\left(f\left(a_{2}, y\right), \omega\right)=f\left(a_{2}, f(y, \omega)\right)=f\left(a_{2}, \omega\right) .
\end{aligned}
$$

Аналогично, $f\left(b_{1}, \omega\right)=f\left(b_{2}, \omega\right)$. Далее, согласно сверхтождеству $(2.4)$ :

$$
\begin{aligned}
f\left(\omega, g\left(a_{1}, b_{1}\right)\right) & =g\left(f\left(\omega, a_{1}\right), f\left(\omega, b_{1}\right)\right) \\
& =g\left(f\left(\omega, a_{2}\right), f\left(\omega, b_{2}\right)\right)=f\left(\omega, g\left(a_{2}, b_{2}\right)\right),
\end{aligned}
$$

т.е. $g\left(a_{1}, b_{1}\right) \widetilde{f}_{I} g\left(a_{2}, b_{2}\right)$.

Если множество $I=\{a\}, a \in Q$, тогда оно $f$-замкнуто для любого $f \in \Sigma$, и соответствуюшую конгруэнцию $\widetilde{f}_{I}$ обозначим через $\widetilde{f}_{a}$. Рассмотрим пересечение $\widetilde{f}=$ $\bigcap_{a \in Q} \widetilde{f}_{a}$. Если $x \widetilde{f} y$, тогда $x \widetilde{f}_{x} y$, и $x \widetilde{f}_{y} y$, т.е. $f(x, x)=f(y, x)$ и $f(x, y)=f(y, y)$,т.е. $x=y$. Итак, конгруэнция $\widetilde{f}$-нулевая, и поскольку алгебра $\mathscr{A}$ подпрямо неразложима, то $\widetilde{f}_{a}$-нулевая конгруэнция для некоторого $a \in Q$. Поэтому $f(x, a)=x$ для любого $x \in Q$ (поскольку $f(x, a) \widetilde{f}_{a} x$ ), т.е. элемент $a$ будет единичньм элементом операции $f \in \Sigma: a=O_{f}$. Итак, каждая операция $f \in \Sigma$ подпрямо неразложимой алгебры $\mathscr{A}$ со сверх тождествами (2.1)-(2.4) обладает единицей $O_{f} \in Q$.

Далее, по любым операциям $f, g \in \Sigma$ определим отношение $f_{g} \subseteq Q \times Q$ следуюшим образом:

$$
x f_{g} y \Longleftrightarrow x=y \text { или } f\left(x, O_{g}\right) \neq O_{g}, \quad f\left(y, O_{g}\right) \neq O_{g} .
$$

Легко доказать, что $f_{g}$ - конгруэнщия для алгебры $(Q ;\{f, g\})$. А именно, $f_{g}$ - отношение эквивалентности, и если $x f_{g} y$ и $z \in Q$, то $f(x, z) f_{g} f(y, z)$ и $g(x, z) f_{g} g(y, z)$, поскольку, если $f\left(x, O_{g}\right) \neq O_{g}$ и $f\left(y, O_{g}\right) \neq O_{g}$, то $f\left(f(x, z), O_{g}\right) \neq$ $O_{g}, f\left(f(y, z), O_{g}\right) \neq O_{g}$, и $f\left(g(x, z), O_{g}\right) \neq O_{g}, f\left(g(y, z), O_{g}\right) \neq O_{g}$. Действительно, пусть $f\left(f(x, z), O_{g}\right)=O_{g}$, тогда

$$
\begin{aligned}
O_{f} & =g\left(O_{f}, O_{g}\right)=g\left(O_{f}, f\left(f(x, z), O_{g}\right)\right) \\
& =f\left(g\left(O_{f}, f(x, z)\right), g\left(O_{f}, O_{g}\right)\right)=f\left(g\left(O_{f}, f(x, z)\right), O_{f}\right) \\
& =g\left(O_{f}, f(x, z)\right)=f\left(g\left(O_{f}, x\right), g\left(O_{f}, z\right)\right) \\
g\left(O_{f}, x\right)= & f\left(g\left(O_{f}, x\right), O_{f}\right)=f\left(g\left(O_{f}, x\right), f\left(g\left(O_{f}, x\right), g\left(O_{f}, z\right)\right)\right) \\
= & f\left(f\left(g\left(O_{f}, x\right), g\left(O_{f}, x\right)\right), g\left(O_{f}, z\right)\right)=f\left(g\left(O_{f}, x\right), g\left(O_{f}, z\right)\right)=O_{f} ; \\
O_{g}= & f\left(O_{g}, O_{f}\right)=f\left(O_{g}, g\left(O_{f}, x\right)\right)=g\left(f\left(O_{g}, O_{f}\right), f\left(O_{g}, x\right)\right) \\
= & g\left(O_{g}, f\left(O_{g}, x\right)\right)=f\left(O_{g}, x\right),
\end{aligned}
$$

что противоречит условию $f\left(x, O_{g}\right) \neq O_{g}$. 
Следовательно, из предположения $f\left(f(y, z), O_{g}\right)=O_{g}$ вытекает равенство $f\left(O_{g}, y\right)=O_{g}$. Если же $f\left(g(x, z), O_{g}\right)=O_{g}$, тогда

$$
\begin{aligned}
O_{f} & =g\left(O_{f}, O_{g}\right)=g\left(O_{f}, f\left(g(x, z), O_{g}\right)\right) \\
& =f\left(g\left(O_{f}, g(x, z)\right), g\left(O_{f}, O_{g}\right)\right) \\
& =f\left(g\left(O_{f}, g(x, z)\right), O_{f}\right)=g\left(O_{f}, g(x, z)\right),
\end{aligned}
$$

т.е. $g\left(g(x, z), O_{f}\right)=O_{f}$, и, следовательно, как и выше, $g\left(O_{f}, x\right)=O_{f}$. Поэтому и $f\left(O_{g}, x\right)=O_{g}$. Опять пришли к противоречию. Аналогично, предположение $f\left(g(y, z), O_{g}\right)=O_{g}$ приводит к противоречию $f\left(O_{g}, y\right)=O_{g}$.

Если для подпрямо неразложимой $T$-алгебры $\mathscr{A}=(Q ; \Sigma)$, где $T=\{2\}$ и $\Sigma=\{f, g\}$, имеем $Q=\left\{O_{f}, O_{g}\right\}$, тогда в случае $f \neq g$ алгебра $\mathscr{A}$ - двухэлементная решетка, а в случае $f=g$ алгебра $\mathscr{A}$ одноэлементна $\left(O_{f}=O_{g}\right)$.

Если же $Q \neq\left\{O_{f}, O_{g}\right\}$, т.е. сушествует $a \in Q$ такое, что $a \neq O_{f}, O_{g}$, тогда $f\left(a, O_{g}\right) \neq O_{g}$. Действительно, предполагая $f\left(a, O_{g}\right)=O_{g}$, получим

$$
\begin{aligned}
y & =g\left(y, O_{g}\right)=g\left(y, f\left(a, O_{g}\right)\right)=f\left(g(y, a), g\left(y, O_{g}\right)\right) \\
& =f(g(y, a), y)=f(y, g(y, a)) .
\end{aligned}
$$

Поэтому если $y\left(\widetilde{f_{a}} \cap \widetilde{g_{a}}\right) x$, тогда

$$
\begin{aligned}
x & =f(x, g(x, a))=f(x, g(y, a))=g(f(x, y), f(x, a))=g(f(x, y), f(y, a)) \\
& =g(f(y, x), f(y, a))=f(y, g(x, a))=f(y, g(y, a))=y,
\end{aligned}
$$

и поскольку алгебра $\mathscr{A}$ - подпрямо неразложима, то $\widetilde{f_{a}}$ или $\widetilde{g_{a}}-$ нулевая конгруэнция, следовательно, $a=O_{f}$ или $a=O_{g}$. Противоречие! Итак, если $a \in Q$ и $a \neq O_{f}, O_{g}$, тогда $f\left(a, O_{g}\right) \neq O_{g}$.

Рассмотрим конгруэнцию

$$
q_{f}^{g}=f_{g} \cap\left(\bigcap_{a \neq O_{f}, O_{g}}\left(\widetilde{f}_{a} \cap \widetilde{g}_{a}\right)\right)
$$

алгебры $\mathscr{A}=(Q ;\{f, g\})$. Поскольку конгруэнщия $\widetilde{f}_{a}$ будет нулевой тогда и только тогда, когда $a=O_{f}$, то конгруэнция $\bigcap_{a \neq O_{f}, O_{g}}\left(\widetilde{f_{a}} \cap \widetilde{g_{a}}\right)$ согласно подпрямой неразложимости алгебры $\mathscr{A}$ не будет нулевой. Далее, справедлива импликация:

$$
x q_{f}^{g} y \longrightarrow x=y
$$

Действительно, если $x=O_{f}$, тогда $f\left(x, O_{g}\right)=f\left(O_{f}, O_{g}\right)=O_{g}$, и, следовательно, $x=y$, поскольку $x f_{g} y$. Аналогично, из условия $x=O_{g}$ получим $f\left(x, O_{g}\right)=$ $f\left(O_{g}, O_{g}\right)=O_{g}$ и опять приходим к равенству $x=y$. Точно так же из условия 
$y=O_{f}\left(y=O_{g}\right)$ вытекает равенство $x=y$. Поэтому при доказательстве импликации можно предположить $x, y \neq O_{f}, O_{g}$. Следовательно, $x\left(\widetilde{f_{x}} \cap \widetilde{g_{x}}\right) y$ и поэтому

$$
\begin{gathered}
f(x, g(x, x))=f(y, g(y, x)), \\
x=f(y, g(y, x)), \\
f(y, x)=f(y, f(y, g(y, x)))=f(y, g(y, x))=x .
\end{gathered}
$$

Аналогично, имеем $x\left(\widetilde{f}_{y} \cap \widetilde{g}_{y}\right) y$ и $f(x, y)=y$. Таким образом, $x=y$. Следовательно, $f_{g}$ - нулевая конгруэнция, и потому множество $Q$ содержит лиш один элемент $a \neq O_{f}, O_{g}$. Итак, либо $|Q|=3$, если $O_{f} \neq O_{g}$, либо $|Q|=2$, если $O_{f}=O_{g}$ (следовательно, и $f=g$ ).

Заметим также, что в случае $|Q|=3$ в алгебре $\mathscr{A}=(Q ;\{f, g\})$ справедливо котождество

$$
X(x, a)=X(a, x)=a
$$

для любого $x \in\left\{O_{f}, O_{g}\right\}$. Действительно, $f\left(a, O_{g}\right) \neq O_{f}$, а если $f\left(a, O_{g}\right)=O_{g}$, тогда возникает противоречие:

$$
a=f\left(a, O_{f}\right)=f\left(a, f\left(O_{g}, a\right)\right)=f\left(O_{g}, a\right)=O_{f} .
$$

Пусть $g\left(a, O_{f}\right)=O_{f}$. Тогда

$$
\begin{aligned}
O_{g} & =f\left(O_{g}, O_{f}\right)=f\left(O_{g}, g\left(a, O_{f}\right)\right)=g\left(f\left(O_{g}, a\right), f\left(O_{g}, O_{f}\right)\right) \\
& =g\left(f\left(O_{g}, a\right), O_{g}\right)=f\left(O_{g}, a\right) .
\end{aligned}
$$

Противоречие! Если же $g\left(a, O_{f}\right)=O_{g}$, тогда

$$
a=g\left(a, O_{g}\right)=g\left(a, g\left(a, O_{f}\right)\right)=g\left(a, O_{f}\right)=O_{g} .
$$

Противоречие!

СледСТвИЕ 2.1. С точностью до изоморфизма единственными неодноэлементными подпрямо неразложимыми Т-алгебрами, где $T=\{2\}$, в сверхмногообразии Т-алгебр, порожденном любой дистрибутивной решеткой (многообразием дистрибутивных решеток), являются: двухәлементная полурешетка, двухәлементная решетка и трехәлементная алгебра $(\{0,1, a\},\{+, \cdot\})$, являющаяся расширением двухәлементной решетки $(\{0,1\},\{+, \cdot\})$ с помощью әлемента а, удовлетворяющего котождеству

$$
X(x, a)=X(a, x)=a
$$

для любого $x \in\{0,1, a\}$. Таким образом, любое сверхтождество многообразия дистрибутивных решеток (любой дистрибутивной решетки) является следствием сверхтождеств (2.1), (2.2), (2.3), (2.4) этого многообразия. 
Теорема 2.3. Если Т-алгебра $\mathscr{A}=(Q ; \Sigma)$, где $T=\{1,2\}$, удовлетворяет сверхтождествам (2.1)-(2.4), а также

$$
\begin{gathered}
F(F(x))=x, \\
X(F(x), y)=X(F(X(x, y)), y), \\
F(X(F(X(x, y)), F(X(x, F(y)))))=x
\end{gathered}
$$

и подпрямо неразложима, тогда множсество унарных операчий алгебры $\mathscr{A}$ одноэлементно, мощность множества ее бинарных операций $\leqslant 2$ u $|Q| \leqslant 2$.

ДокАЗАТЕльство. Для любой бинарной операции $f \in \Sigma$ и для любого непустого $f$-замкнутого подмножества $I \in Q$ отношение $\widetilde{f}_{I}$, определенное при доказательстве теоремы 2.2, является конгруэнщией и для рассматриваемой $T$-алгебры. Остается лишь добавить, что для любой унарной операции $F \in \Sigma$

$$
a \widetilde{f}_{I} b \rightarrow F(a) \widetilde{f}_{I} F(b) .
$$

Действительно, если $f(a, x)=f(b, x), x \in I$, тогда согласно сверхтождеству $(2.15)$ получим:

$$
f(F(a), x)=f(F(f(a, x)), x)=f(F(f(b, x)), x)=f(F(b), x),
$$

т.е. $f(a) \widetilde{f}_{I} F(b)$.

Поэтому, как и в доказательстве теоремы 2.2 , заключаем, что любая бинарная операция $f \in \Sigma$ подпрямо неразложимой $T$-алгебры $\mathscr{A}=(Q ; \Sigma)$, где $T=\{1,2\}$, со сверхтождествами (2.1)-(2.4), (2.15), обладает единищей $O_{f} \in Q$. Очевидно,

$$
I \subseteq J \longrightarrow \widetilde{f_{I}} \subseteq \widetilde{f_{J}} .
$$

$f$-замкнутое подмножество $I \subseteq Q$ назьвается простьц, если

$$
f(x, y) \in I \longrightarrow x, y \in I .
$$

Очевидно, $Q$ является $f$-замкнутьм и простым для любой бинарной операции $f \in \Sigma$, и пересечение $f$-замкнутых и простых подмножеств есть $f$-замкнутое и простое подмножество.

Для любого элемента $a \in Q$ и любой бинарной операции $f \in \Sigma$ непустое подмножество

$$
(a)_{f}=\{x \in Q \mid f(a, x)=a\}
$$

будет $f$-замкнутым и простьм. Действительно, если $x, y \in(a)_{f}$, т.е. $f(a, x)=a$ и $f(a, y)=a$, то

$$
f(a, f(x, y))=f(f(a, x), y)=f(a, y)=a,
$$

т.е. $f(x, y) \in(a)_{f}$. Если же $f(x, y) \in(a)_{f}$, то $x, y \in(a)_{f}$. Например,

$$
\begin{aligned}
f(a, x) & =f(f(a, f(x, y)), x)=f(a, f(f(y, x), x)) \\
& =f(a, f(y, f(x, x)))=f(a, f(y, x))=f(a, f(x, y))=a .
\end{aligned}
$$


Кроме того, очевидно, что

$$
(a)_{f}=\left\{O_{f}\right\} \Longleftrightarrow a=O_{f},
$$

и конгруэнция $\widetilde{f}_{(a)_{f}}$ будет нулевой тогда и только тогда, когда $a=O_{f}$.

Если $I$ и $J-f$-замкнутые и простые подмножества, то

$$
\widetilde{f}_{I} \subseteq \tilde{f}_{J} \longrightarrow I \subseteq J
$$

поскольку при $\widetilde{f}_{I} \subseteq \widetilde{f}_{J}$ имеем:

$$
\begin{aligned}
I & =\left\{x \in Q \mid \exists a, b \in I, f(x, a)=f\left(O_{f}, b\right)\right\} \\
& =\left\{x \in Q \mid x \widetilde{f}_{I} O_{f}\right\} \subseteq\left\{x \in Q \mid x \widetilde{f}_{J} O_{f}\right\} \\
& =\left\{x \in Q \mid \exists c, d \in J, f(x, c)=f\left(O_{f}, d\right)\right\}=J .
\end{aligned}
$$

Следовательно,

$$
\widetilde{f}_{(a)_{f}} \cap \widetilde{f}_{(b)_{f}}=\widetilde{f}_{(a)_{f} \cap(b)_{f}} .
$$

Пусть $|Q| \geqslant 3$, и пусть $F, f$ - фиксированные унарная и бинарная операции из $\Sigma$. Следовательно, существует $a \in Q, a \neq O_{f}, F\left(O_{f}\right)$. Тогда, согласно сверхтождеству (2.14) $F(a) \neq O_{f}$. Поэтому $(a)_{f} \neq\left\{O_{f}\right\},(F(a))_{f} \neq\left\{O_{f}\right\}$. Если же $x \in(a)_{f} \cap$ $(F(a))_{f}$, то $f(a, x)=a, f(F(a), x)=F(a)$ и

$$
x \stackrel{(2.16)}{=} F(f(F(f(x, a)), F(f(x, F(a)))))=F(f(F(a), F(F(a))))=F(f(F(a), a)) .
$$

Следовательно, пересечение $(a)_{f} \cap(F(a))_{f}$ одноэлементно, поэтому

$$
(a)_{f} \cap(F(a))_{f}=\left\{O_{f}\right\}
$$

и согласно (2.17) пересечение $\widetilde{f}_{(a)_{f}} \cap \widetilde{f}_{(F(a))_{f}}$ двух ненулевых конгруэнций - нулевая конгруэнция. Противоречие! Итак, $|Q| \leqslant 2$.

Если $|Q|=2$, тогда существуют всего две бинарные операции, удовлетворяюшие условиям (2.1), (2.2), и столько же унарных операций, удовлетворяюших условию (2.14). Однако тождественное отображение $\varepsilon_{Q}$ не удовлетворяет сверхтождествy $(2.16)$ :

$$
\begin{aligned}
x & =\varepsilon_{Q} X\left(\varepsilon_{Q} X(x, y), \varepsilon_{Q} X\left(x, \varepsilon_{Q}(y)\right)\right) \\
& =\varepsilon_{Q} X\left(\varepsilon_{Q} X(y, x), \varepsilon_{Q} X\left(y, \varepsilon_{Q}(x)\right)\right)=y .
\end{aligned}
$$

СЛЕДСТвИЕ 2.2. С точностью до изоморфизма единственными неодноэлементными подпрямо неразложимыми $T$-алгебрами, где $T=\{1,2\}$, в сверхмногообразии $T$-алгебр, порожденном любой булевой алгеброй (многообразием булевых алгебр), являются двухәлементная булева алгебра и ее $\left\{+,{ }^{\prime}\right\}$-редукт. Таким образом, любое сверхтождество многообразия булевых алгебр (любой булевой алгебры) является следствием сверхтождеств (2.1), (2.2), (2.3), (2.4), (2.14), (2.15), (2.16) этого многообразия. 
Теорема 2.4 [21]. Если T-алгебра $\mathscr{A}=(Q ; \Sigma)$, где $T=\{2\}$, удовлетворяет сверхтождествам (2.1)-(2.3), а также

$$
X(Y(X(x, y), z), Y(y, z))=Y(X(x, y), z)
$$

u подпрямо неразложима, тогда мощность $|\Sigma| \leqslant 2$, и поэтому

1) любое сверхтождество многообразия всех решеток является следствием сверхтохсдеств (2.1), (2.2), (2.3), (2.18) этого многообразия;

2) любое сверхтождество многообразия всех модулярных решеток является следствием сверхтождеств (2.1), (2.2), (2.3), (2.18), (1.2) этого многообразия.

Об исследованиях сверхтождеств в полиномиальных (термальных) алгебрах см. в $[42]-[53]$.

\section{$\S$ 3. Категория арифметически однотипных систем. Неклассическая семантика языка 2-го порядка}

Под арифметическим типом алгебраической системы (кратко - системы) $\mathscr{A}=$ $(Q ; \Sigma, \Omega)$ понимается упорядоченная пара $\left(T_{1}, T_{2}\right)$ подмножеств множества всех натуральных чисел:

$$
\begin{aligned}
& T_{1}=\{|A| \mid A \in \Sigma\} \subseteq N, \\
& T_{2}=\{|P| \mid P \in \Omega\} \subseteq N,
\end{aligned}
$$

где $|P|$ (соответственно $|A|)$ - арность предиката $P$ (операции $A$ ). Алгебраическая система с арифметическим типом $\left(T_{1}, T_{2}\right)$ называется $\left(T_{1}, T_{2}\right)$-системой. Если $\Sigma=\varnothing$, то $\mathscr{A}$ назьвается $T_{2}$-моделью. Две системы назьваются арифметически однотипными, если их арифметические типы равны.

Пусть $\mathscr{A}=\langle Q ; \Sigma, \Omega\rangle$ и $\mathscr{A}^{\prime}=\left\langle Q^{\prime} ; \Sigma^{\prime}, \Omega^{\prime}\right\rangle$ - две арифметически однотипные системы. ${ }^{7}$ Тройку отображений $(\alpha, \widetilde{\beta}, \widetilde{\gamma})$, где $\alpha: Q \rightarrow Q^{\prime}, \widetilde{\beta}: \Sigma \rightarrow \Sigma^{\prime}, \widetilde{\gamma}: \Omega \rightarrow \Omega^{\prime}$, назовем гомоморфизмом из системы $\mathscr{A}$ в систему $\mathscr{A}^{\prime}$ (обозначение: $(\alpha, \widetilde{\beta}, \widetilde{\gamma}): \mathscr{A} \Rightarrow \mathscr{A}^{\prime}$ ), если $\widetilde{\beta}$ и $\widetilde{\gamma}$ сохраняют арность и

$$
\begin{gathered}
\alpha A\left(x_{1}, \ldots, x_{n}\right)=[\widetilde{\beta}(A)]\left(\alpha x_{1}, \ldots, \alpha x_{n}\right), \\
P\left(y_{1}, \ldots, y_{m}\right)=И \Rightarrow[\widetilde{\gamma}(P)]\left(\alpha y_{1}, \ldots, \alpha y_{m}\right)=И,
\end{gathered}
$$

где $A \in \Sigma, P \in \Omega,|A|=n,|P|=m, x_{1}, \ldots, x_{n}, y_{1}, \ldots, y_{m} \in Q$.

Арифметически однотипные системы и их гомоморфизмы $(\alpha, \widetilde{\beta}, \widetilde{\gamma}$ ) (в качестве морфизмов) образуют категорию. В дальнейшем понятия подсистемы, ядра, конгруэции, фактор-системы, прямого произведения систем, фильтрованного произведения и ультрапроизведения систем понимаются в смысле этой категории.

Пусть заданы некоторьй язык $\mathscr{K}$ второго порядка [1], [2] и произвольные подмножества $T_{1}, T_{2}$ множества натуральных чисел $\mathbb{N}$. Будем говорить, что формула $F$

\footnotetext{
${ }^{7}$ Для простоты рассматриваются алгебраические системы без нульарных операций и предикатов.
} 
этого языка имеет арифметический тип $\left(T_{1}, T_{2}\right)$ или является $\left(T_{1}, T_{2}\right)$-формулой, если множество арностей всех функциональных (предикатных) переменных, участвующих в этой формуле, содержится в $T_{1}$ (соответственно в $T_{2}$ ). Значения и истинность $\left(T_{1}, T_{2}\right)$-формул языка $\mathscr{K}$ здесь определяются на алгебраической системе $\mathscr{A}=$ $\langle Q ; \Sigma, \Omega\rangle$ арифметического типа $\left(T_{1}, T_{2}\right)$ как значения и истинность формул с ограниченними предикатными (функциональными) кванторами $(\forall X),(\exists X)$, означающими: "для каждого значения $X=P \in \Omega$ (соответственно $X=A \in \Sigma$ ) соответствуюшей арности", "существует значение $X=P \in \Omega$ (соответственно $X=A \in \Sigma$ ) соответствуюшей арности". Значения соответствуюших арностей для свободных предикатных и функциональных переменных также берутся из системы $\mathscr{A}$, точнее, из $\Omega$ и $\Sigma$ соответственно.

Абсолютно-замкнутая формула второго порядка назьвается предложением (свойством) второго порядка. Прямое произведение $\left(T_{1}, T_{2}\right)$-систем, вообще говоря, не сохраняет свойств второго порядка.

Рассмотрим фильтрованные произведения в рассматриваемой категории систем. Пусть $\mathscr{A}_{i}=\left(Q_{i} ; \Sigma_{i}, \Omega_{i}\right), i \in I,-$ системы арифметического типа $\left(T_{1}, T_{2}\right)$, и пусть $\widehat{\mathscr{A}}=(\widehat{Q} ; \widehat{\Sigma}, \widehat{\Omega})$ - прямое произведение этих $\left(T_{1}, T_{2}\right)$-систем, определяемое аналогично прямым произведениям Т-алгебр [8]. Пусть $\mathscr{D}$ - какой-нибудь фильтр над $I$. Тройка отношений эквивалентности $\left(\equiv_{\mathscr{D}}, \sim_{\mathscr{D}}, \cong_{\mathscr{D}}\right)$, где $\equiv_{\mathscr{D}}, \sim_{\mathscr{D}}$ и $\cong_{\mathscr{D}}$ определяются аналогично:

$$
\begin{aligned}
f \equiv_{\mathscr{D}} g & \Longleftrightarrow\{\alpha \in I \mid f(\alpha)=g(\alpha)\} \in \mathscr{D}, \quad f, g \in \widehat{Q}, \\
F \sim_{\mathscr{D}} G & \Longleftrightarrow\{\alpha \in I \mid F(\alpha)=G(\alpha)\} \in \mathscr{D}, \quad F, G \in \widehat{\Sigma}, \\
P \cong_{\mathscr{D}} R & \Longleftrightarrow\{\alpha \in I \mid P(\alpha)=R(\alpha)\} \in \mathscr{D}, \quad P, R \in \widehat{\Omega},
\end{aligned}
$$

будет конгруэнцией $\left(T_{1}, T_{2}\right)$-системы $\hat{\mathscr{A}}$, т.е. $\left(\equiv \mathscr{D}, \sim_{\mathscr{D}}\right)$ - конгруэнция $T_{1}$-алгебры $\langle\widehat{Q} ; \widehat{\Sigma}\rangle$ в смысле [8]. Тройка $\langle\widehat{Q} / \mathscr{D} ; \widehat{\Sigma} / \mathscr{D}, \widehat{\Omega} / \mathscr{D}\rangle$, состояшая из фактор-множеств $\widehat{Q}$, $\widehat{\Sigma}$ и $\widehat{\Omega}$ по отношениям эквивалентности $\equiv \mathscr{D}, \sim_{\mathscr{D}}$ и $\cong_{\mathscr{D}}$, естественно преврашается в $\left(T_{1}, T_{2}\right)$-систему. Действительно, пусть $f \mathscr{D}$-класс эквивалентности элемента $f \in \widehat{Q}$ по отношению $\equiv_{\mathscr{D}},[A] \mathscr{D}-$ класс эквивалентности операции $A \in \widehat{\Sigma}$ по отношению $\sim \mathscr{D}$, и $[R] \mathscr{D}$ - класс эквивалентности отношения $R \in \widehat{\Omega}$ по эквивалентности $\cong_{\mathscr{D}}$. Каждый элемент $[A] \mathscr{D} \in \widehat{\Sigma} / \mathscr{D}$ определяет операцию на множестве $\widehat{Q} / \mathscr{D}$ той же арности, что и операция $A \in \widehat{\Sigma}$ :

$$
[A] \mathscr{D}\left(f_{1} \mathscr{D}, \ldots, f_{n} \mathscr{D}\right)=\left[A\left(f_{1}, \ldots, f_{n}\right)\right] \mathscr{D},|A|=n,
$$

корректность которой следует из определения конгруэции. Имеем:

$$
[A] \mathscr{D}\left(f_{1} \mathscr{D}, \ldots, f_{n} \mathscr{D}\right)=f \mathscr{D} \Longleftrightarrow\left\{\alpha \in I \mid A(\alpha)\left(f_{1}(\alpha), \ldots, f_{n}(\alpha)\right)=f(\alpha)\right\} \in \mathscr{D} .
$$

Каждый элемент $[R] \mathscr{D} \in \widehat{\Omega} / \mathscr{D}$ определяет предикат на множестве $\widehat{Q} / \mathscr{D}$ той же арности, что и предикат $R \in \widehat{\Omega}$ :

$$
[R] \mathscr{D}\left(f_{1} \mathscr{D}, \ldots, f_{m} \mathscr{D}\right)=И \Longleftrightarrow\left\{\alpha \in I \mid R(\alpha)\left(f_{1}(\alpha), \ldots, f_{m}(\alpha)\right)=И\right\} \in \mathscr{D},|R|=m,
$$


корректность которого следует из определения фильтра. Действительно, если $g_{i} \in f_{i} \mathscr{D}, i=1, \ldots, m, P \in[R] \mathscr{D}$ и

$$
\begin{aligned}
& I_{0}=\{\alpha \in I \mid R(\alpha)=P(\alpha)\} \in \mathscr{D}, \\
& I_{j}=\left\{\alpha \in I \mid f_{j}(\alpha)=g_{j}(\alpha)\right\} \in \mathscr{D}, \quad j=1, \ldots, m,
\end{aligned}
$$

TO

$$
\begin{aligned}
& \{\alpha \in I \mid P(\alpha) \\
& \left.\left(g_{1}(\alpha), \ldots, g_{m}(\alpha)\right)=И\right\} \\
& \supseteq I_{0} \cap I_{1} \cap \cdots \cap I_{m} \cap\left\{\alpha \in I \mid R(\alpha)\left(f_{1}(\alpha), \ldots, f_{m}(\alpha)\right)=И\right\} \in \mathscr{D} .
\end{aligned}
$$

Полученная $\left(T_{1}, T_{2}\right)$-система $\langle\widehat{Q} / \mathscr{D} ; \widehat{\Sigma} / \mathscr{D}, \widehat{\Omega} / \mathscr{D}\rangle$ назьвается фильтрованным (по фильтру $\mathscr{D})$ произведением $\left(T_{1}, T_{2}\right)$-систем $\mathscr{A}_{i}, i \in I$, и обозначается через $\prod_{i \in I} \mathscr{A}_{i} / \mathscr{D}$. Если фильтр $\mathscr{D}$ - ультрафильтр над $I$, то фильтрованное произведение $\left(T_{1}, T_{2}\right)$-систем называется ультрапроизведением $\left(T_{1}, T_{2}\right)$-систем.

Запись $\mathscr{A} \models \mathscr{F}$ означает, что $\left(T_{1}, T_{2}\right)$-формула $\mathscr{F}$ или ее соответствуюшее значение истинна на $\left(T_{1}, T_{2}\right)$-системе $\mathscr{A}$.

Через $\mathscr{F}\left(x_{1}, \ldots, x_{n}, X_{1}, \ldots, X_{m}, S_{1}, \ldots, S_{k}\right)$ обозначим $\left(T_{1}, T_{2}\right)$-формулу, свободные предметные переменные которой содержатся среди $x_{1}, \ldots, x_{n}$, а свободные функшиональные (предикатные) переменные - среди $X_{1}, \ldots, X_{m}$, (соответственно среди $\left.S_{1}, \ldots, S_{k}\right)$.

ОПРЕДЕЛЕНИЕ 3.1. $\left(T_{1}, T_{2}\right)$-формула $\mathscr{F}\left(x_{1}, \ldots, x_{n}, X_{1}, \ldots, X_{m}, S_{1}, \ldots, S_{k}\right)$ называется фильтруюшейся по фильтру $\mathscr{D}$ (над $I)$, если для любого множества $\left(T_{1}, T_{2}\right)$-систем $\mathscr{A}_{i}=\left\langle Q_{i} ; \Sigma_{i}, \Omega_{i}\right\rangle, i \in I$, и любых $a_{1}, \ldots, a_{n} \in \widehat{Q}, F_{1}, \ldots, F_{m} \in \widehat{\Sigma}, P_{1}, \ldots, P_{k} \in \widehat{\Omega}$ соответствуюших арностей имеет место

$$
\begin{aligned}
\left\{\alpha \in I \mid \mathscr{A}_{\alpha} \models\right. & \left.\mathscr{F}\left(a_{1}(\alpha), \ldots, a_{n}(\alpha), F_{1}(\alpha), \ldots, F_{m}(\alpha), P_{1}(\alpha), \ldots, P_{k}(\alpha)\right)\right\} \in \mathscr{D} \\
& \Longleftrightarrow \prod_{i \in I} \mathscr{A}_{i} / \mathscr{D}=\mathscr{F}\left(a_{1} \mathscr{D}, \ldots, a_{n} \mathscr{D},\left[F_{1}\right] \mathscr{D}, \ldots,\left[F_{m}\right] \mathscr{D},\left[P_{1}\right] \mathscr{D}, \ldots,\left[P_{k}\right] \mathscr{D}\right) .
\end{aligned}
$$

В рассматриваемой категории $\left(T_{1}, T_{2}\right)$-систем и их гомоморфизмов $(\alpha, \widetilde{\beta}, \widetilde{\gamma})$ существование фильтрованных произведений и ультрапроизведений приводит к формулировкам основных теоретико-модельных результатов для формул второго порядка с ограниченными функциональными и предикатными кванторами.

ТЕОрема 3.1 (Лося для формул второго порядка с ограниченными кванторами). Произвольная $\left(T_{1}, T_{2}\right)$-формула $\mathscr{F}\left(x_{1}, \ldots, x_{n}, X_{1}, \ldots, X_{m}, S_{1}, \ldots, S_{k}\right)$ является фильтрующейся (в смысле определения 3.1) по любому ультрафильтру.

ДокАЗАТЕльство. Применяется стандартная индукция по длине формулы $\mathscr{F}$. Пусть $D$ - ультрафильтр над множеством $I$. Для атомных формул утверждение верно. Далее, допуская, что утверждение верно для формул $\mathscr{F}_{1}, \mathscr{F}_{2}$, доказьваем его для формул $\mathscr{F}_{1} \& \mathscr{F}_{2}, \neg \mathscr{F}_{1},(\exists x) \mathscr{F}_{1},(\exists X) \mathscr{F}_{1}$ и $(\exists S) \mathscr{F}_{1}$ (остальные логические операции выражаются через перечисленные), где $x$ - предметная, $X$ - функциональная и $S$ предикатная переменные. 
СЛЕДСТВИЕ 3.1. Если предложсение второго порядка и арифметического типа $\left(T_{1}, T_{2}\right)$ истинно в каждой $\left(T_{1}, T_{2}\right)$-системе $\mathscr{A}_{i}, i \in I$, то оно истинно и в любом ультрапроизведении этих $\left(T_{1}, T_{2}\right)$-систем.

Алгебраическую систему $\mathscr{A}$ арифметического типа $\left(T_{1}, T_{2}\right)$ будем назьвать $\left(T_{1}, T_{2}\right)$-моделью множества $Г$ предложений второго порядка и арифметического типа $\left(T_{1}, T_{2}\right)$, если $\mathscr{A} \models \mathscr{F}$ для всех $\mathscr{F} \in \Gamma$.

СлЕДСТВИЕ 3.2. Множсество Г предложений второго порядка и арифметического типа $\left(T_{1}, T_{2}\right)$ имеет $\left(T_{1}, T_{2}\right)$-модель тогда и только тогда, когда всякое его конечное подмножество $\Gamma_{0} \subseteq \Gamma$ имеет $\left(T_{1}, T_{2}\right)$-модель.

Пусть $\Gamma_{1}, \Gamma_{2}$ - множества предложений второго порядка и арифметического типа $\left(T_{1}, T_{2}\right)$. Будем говорить, что $\Gamma_{2}$ является следствием $\Gamma_{1}$, если любая $\left(T_{1}, T_{2}\right)$-модель для $\Gamma_{1}$ является $\left(T_{1}, T_{2}\right)$-моделью и для $\Gamma_{2}$. Множества $\Gamma_{1}, \Gamma_{2}$ назьваются эквивалентными (равносильными), если $\Gamma_{2}$ является следствием $\Gamma_{1}$, и наоборот.

СлЕДСТВИЕ 3.3. Если множество Г предложений второго порядка и арифметического типа $\left(T_{1}, T_{2}\right)$ равносильно множеству $\{\mathscr{F}\}$, состоящему из одного $\left(T_{1}, T_{2}\right)$-предложсения второго порядка, то существует конечное подмножество $\Gamma_{0} \subseteq \Gamma$, равносильное $\{\mathscr{F}\}$.

$\left(T_{1}, T_{2}\right)$-формула $F$ со свободными предметньми, функциональными и (или) предикатными переменными называется вьполнимой в $\left(T_{1}, T_{2}\right)$-системе $\mathscr{A}$, если существуют такие значения из $\mathscr{A}$ для всех входящих в $F$ свободных переменных, при которых формула $F$ становится истинной в $\mathscr{A}$. Совокупность $\left(T_{1}, T_{2}\right)$-формул $\mathscr{F}$ назьвается вьполнимой в $\left(T_{1}, T_{2}\right)$-системе $\mathscr{A}$, если сушествуют такие значения из $\mathscr{A}$ для всех входящих в элементы $\mathscr{F}$ свободных переменных, при которых формулы из $\mathscr{F}$ становятся истинными в $\mathscr{A}$. Совокупность $\left(T_{1}, T_{2}\right)$-формул назьвается вьполнимой, если сушествует $\left(T_{1}, T_{2}\right)$-система, в которой выполнима эта совокупность.

Пусть $n \in \mathbb{N}$. Совокупность $\left(T_{1}, T_{2}\right)$-формул $Г$ назьвается $n$-вьполнимой, если существует $\left(T_{1}, T_{2}\right)$-система, в которой вьполнимо любое подмножество $\mathscr{F} \subseteq \Gamma$ порядка $\leqslant n$.

СлЕДСТВИЕ 3.4. Если выполнима (п-выполнима) каждая конечная часть бесконечной совокупности $\left(T_{1}, T_{2}\right)$-формул, то выполнима (п-вьполнима) и вся за-

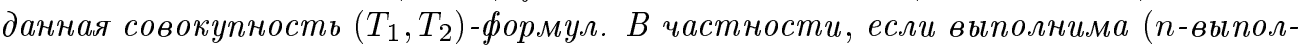
нима) каждая конечная часть бесконечной совокупности $\left(T_{1}, T_{2}\right)$-формул язька 1-го порядка, то вьполнима (п-выполнима) и вся заданная совокупность $\left(T_{1}, T_{2}\right)$-формул $(n=1,2, \ldots)$.

ЗАмЕчАнИЕ. Нетрудно ввести понятие категории с ультрапроизведениями и тем самым прийти к теореме компактности в категориях. Подобное замечание справедливо и для теоремы о полноте в категориях со свободньци объектами.

\section{$\S$ 4. Нетривиальные сверхтождества ассоциативности и дистрибутивности в обратимых и близких к ним алгебрах}

В настояшем параграфе рассматриваются $T$-сверхтождества при $T=\{2\}$. 
Бинарная алгебра $(Q ; \Sigma)$ называется $q$-алгеброй, если в $\Sigma$ существует обратимая операция, т.е. $Q(A)$ - квазигруппа для некоторого $A \in \Sigma$. Бинарная алгебра $(Q ; \Sigma)$ называется $e$-алгеброй, если в $\Sigma$ существует операция с единицей.

ОпредЕлениЕ 4.1. Функциональная переменная $X$ назьвается сингулярной в сверхтождестве $w_{1}=w_{2}$, если в нем $X$ встречается всего один раз и справедливо хотя бы одно из следующих условий:

а) в подслове $w=X\left(\omega_{1}, \omega_{2}\right)$ существуют предметные переменные $x \in\left[\omega_{1}\right]$ и $y \in$ $\left[\omega_{2}\right]$ такие, что каждая из них в подслове $w$ встречается всего один раз;

б) подслово $w=X\left(\omega_{1}, \omega_{2}\right)$ имеет вид $X\left(\omega_{1}, x\right)$ или $X\left(x, \omega_{2}\right)$, и существует предметная переменная $y \in[w]$, отличная от $x$ и встречающаяся в подслове $w$ всего один раз, где $[w]$ - множество предметных переменных в слове $w$.

Функциональная переменная $X$ называется строго сингулярной в сверхтождестве $w_{1}=w_{2}$, если в нем $X$ встречается всего один раз и в подслове $X\left(\omega_{1}, \omega_{2}\right)$ слова $w_{i}$ $(i=1$ или 2$)$ сушествуют предметные переменные $x \in\left[\omega_{1}\right]$ и $y \in\left[\omega_{2}\right]$ такие, что каждая из них в слове $w_{i}$ встречается всего один раз.

Лемма 4.1 [8]. В нетривиальной q-алгебре не может выполняться сверхтождество с сингулярной функииональной переменной. В нетривиальной е-алгебре не может выполняться сверхтождество со строго сингулярной функииональной переменной.

ДокАЗАТЕльство. Предположим обратное. Пусть в нетривиальной $q$-алгебре $\mathscr{A}=\langle Q ; \Sigma\rangle$ с обратимой операцией $A \in \Sigma$ выполняется сверхтождество

$$
w_{1}=w_{2}
$$

с сингулярной функциональной переменной $X$. Задавая для функциональной переменной $X$ два различных значения $A_{1} \neq A_{2}$, где $A_{1}, A_{2} \in \Sigma$, и в обоих случаях давая всем остальным функциональным переменным из сверхтождества (4.0) равное значение $A \in \Sigma$, где $Q(A)$ - квазигруппа, получаем два тождества:

$$
w_{1}^{\prime}=w_{2}^{\prime}, \quad w_{1}^{\prime \prime}=w_{2}^{\prime \prime}
$$

Пусть для определенности $X$ содержится в $w_{1}$, тогда выражения $w_{2}^{\prime}$ и $w_{2}^{\prime \prime}$, будучи идентичными, совпадают, т.е. $w_{2}^{\prime}=w_{2}^{\prime \prime}$, и поэтому $w_{1}^{\prime}=w_{1}^{\prime \prime}$.

В $w_{1}^{\prime}$ и $w_{1}^{\prime \prime}$ все операции совпадают с $A$, кроме $A_{1}, A_{2}$. Поскольку $Q(A)$ - квазигруппа, то, проделав все сокращения, получаем тождество

$$
A_{1}\left(\omega_{1}, \omega_{2}\right)=A_{2}\left(\omega_{1}, \omega_{2}\right) .
$$

Пусть подслово $X\left(\omega_{1}, \omega_{2}\right)$ удовлетворяет условию а) определения 4.1. Кроме предметных переменных $x$ и $y$, придадим каждой предметной переменной произвольное, но фиксированное значение из $Q$. Тогда $\omega_{1}$ преврашается в $\lambda(x)$, а $\omega_{2}-$ в $\mu(y)$, где $\lambda$ и $\mu-$ 
подстановки множества $Q$, поскольку они являются произведениями транслящий для квазигруппы $Q(A)$. Таким образом, имеем

$$
A_{1}(\lambda x, \mu y)=A_{2}(\lambda x, \mu y)
$$

Отсюда, поскольку $\lambda$ и $\mu$ - подстановки, получаем равенство $A_{1}=A_{2}$, что противоречит выбору $A_{1}, A_{2} \in \Sigma$.

Аналогично рассматривается случай б).

При доказательстве второй части леммы все предметные переменные, кроме $x, y$, заменяются единицей.

СлЕДСТВИЕ 4.1. Если в нетривиальной q-алгебре выполняется нетривиальное сверхтождество ассочиативности, то оно может быть только функционального ранга 2 и одного из следующих видов:

$\left((\operatorname{ass})_{1}\right)$

$\left((\text { ass })_{2}\right)$

$\left((\text { ass })_{3}\right)$

$$
\begin{aligned}
& X(x, Y(y, z))=Y(X(x, y), z) \\
& X(x, Y(y, z))=X(Y(x, y), z), \\
& X(x, X(y, z))=Y(Y(x, y), z),
\end{aligned}
$$

причем в классе q-алгебр из сверхтождества (ass) $)_{3}$ вытекает сверхтождество (ass) ${ }_{2}$, а из сверхтождества (ass) 2 вытекает сверхтождество (ass) 1 . В q-алгебре со сверхтождеством (ass) $)_{1}$ выполняется сверхтождество (ass) 2 , если и только если в ней выполняется сверхтождество

$$
X(x, Y(y, x))=X(Y(x, y), x)
$$

а в q-алгебре со сверхтождеством (ass) 2 выполняется сверхтожсдество (ass) ${ }_{3}$, если и только если в ней выполняется котождество

$$
X(a, X(b, c))=Y(Y(a, b), c)
$$

q-алгебра с нетривиальным сверхтождеством ассоциативности оказывается обратимой.

ДокАЗАТЕльство. Если функциональньй ранг сверхтождества ассоциативности равен 4 или 3, тогда в нем существует сингулярная функциональная переменная, поэтому согласно лемме 4.1 такое сверхтождество не может вьполняться в нетривиальной $q$-алгебре. Если же функциональный ранг равен 2 , то возможны лишь три случая, отмеченные в следствии.

Если в $q$-алгебре $(Q ; \Sigma)$ вьполняется одно из сверхтождеств (ass $)_{1},(\text { ass })_{2},(\text { ass })_{3}$, тогда в $(Q ; \Sigma)$ должно вьполняться и тривиальное сверхтождество ассощиативности

$$
X(x, X(y, z))=X(X(x, y), z)
$$

другими словами, все операции из $\Sigma$ - полугрупповые, поэтому $Q(\cdot)$ - группа для некоторого $A \in \Sigma$. Докажем, что если в $q$-алгебре $(Q ; \Sigma)$ выполняется сверхтождество (ass) 1 , тогда каждая операция $A_{i} \in \Sigma$ определяется по правилу 
$A_{i}(x, y)=x \cdot t_{i} \cdot y$, где $t_{i}$ - некоторый элемент из $Q$, зависяший от $A_{i} \in \Sigma$. В самом деле, полагая в (ass) 1 X $A_{i}, Y=(\cdot)$ и $y=e$ - единица группы $Q(\cdot)$, получим $A_{i}(x, z)=A_{i}(x, e) \cdot z=\lambda_{i}(x) \cdot z$, где $\lambda_{i}(x)=A_{i}(x, e)$. Полагая в сверхтождестве (ass) 1 $X=(\cdot)$ и $Y=A_{i}$, получаем $x \cdot\left(\lambda_{i} y \cdot z\right)=\lambda_{i}(x \cdot y) \cdot z, x \cdot \lambda_{i} y=\lambda_{i}(x \cdot y), x \cdot \lambda_{i} e=\lambda_{i}(x)$, $x \cdot t_{i}=\lambda_{i}(x)$, где $t_{i}=\lambda_{i} e$. Следовательно, $A_{i}(x, y)=x \cdot t_{i} \cdot y$. Непосредственной проверкой убеждаемся и в обратном: если каждая операция $A_{i} \in \Sigma$ определяется по правилу $A_{i}(x, y)=x \cdot t_{i} \cdot y$, где $Q(\cdot)$ - группа и $t_{i} \in Q$, тогда в алгебре $(Q ; \Sigma)$ вьполняется сверхтождество (ass) 1 .

Рассмотрим теперь $q$-алгебру $(Q ; \Sigma)$ со сверхтождеством $(\text { ass })_{2}$. Как и в предыдушем случае, полагая $X=(\cdot) \in \Sigma, Y=A_{i} \in \Sigma$ и $z=e$, получим $A_{i}(x, y)=x \cdot \lambda_{i}(y)$, где $\lambda_{i}(y)=A_{i}(y, e)$. Далее, находим $\lambda_{i}(y)=y \cdot t_{i}$, откуда $A_{i}(x, y)=x \cdot y \cdot t_{i}$ и $x \cdot y \cdot z \cdot t_{i}=x \cdot y \cdot t_{i} \cdot z$, т.е. элемент $t_{i}$ принадлежит центру групшы $Q(\cdot)$. Непосредственная проверка показьвает и обратное, что в такой алгебре действительно вьполняется сверхтождество (ass) 2 .

Аналогично доказьвается, что в $q$-алгебре $(Q ; \Sigma)$ выполняется сверхтождество $(\mathrm{ass})_{3}$ тогда и только тогда, когда существует группа $Q(\cdot)$ такая, что каждая операция $A_{i} \in \Sigma$ определяется по правилу $A_{i}(x, y)=x \cdot t_{i} \cdot y$, где $t_{i}$ - центральньй элемент второго порядка групшы $Q(\cdot)$.

СЛЕДСТВИЕ 4.2. Если в нетривиальной е-алгебре выполняется нетривиальное сверхтождество ассочиативности, то оно может быть только функционального ранга 2 и имеет вид одного из сверхтождеств (ass) $1,(\mathrm{ass})_{2}$, (ass) $)_{3}$, причем в классе е-алгебр из сверхтождества (ass) ${ }_{3}$ вытекает сверхтождество (ass) ${ }_{2}$, а из сверхтождества (ass) $)_{2}$ вытекает сверхтождество (ass) ${ }_{1}$.

Доказательство следствия 4.2 подобно доказательству следствия 4.1.

Без доказательства отметим следуюший результат, охватьвающий классические результаты Брака-Хьюза [54], Алберта [55].

ТеОРема 4.1 [9]. Если е-алгебра изотопна бинарной алгебре с нетривиальнылм сверхтождеством ассочиативности, тогда они изомофны.

Сверхтождества ассоциативности в обратимых алгебрах впервые рассмотрены в [10]. См. также [56], [57].

ПримеР 4.1. Пусть $Q(+, \cdot)$ - нильпотентное ассоциативное кольцо индекса 5 , $A_{a}(x, y)=x \cdot a \cdot y, a \in Q$, и пусть $\Sigma=\left\{A_{a} \mid a \in Q\right\}$, тогда в алгебре $(Q ; \Sigma)$ вьполняются следуюшие нетривиальные сверхтождества ассоциативности, левой и правой дистрибутивности:

$$
\begin{aligned}
& X(x, Y(y, z))=U(V(x, y), z), \\
& X(x, Y(y, z))=U(V(x, y), Z(x, z)), \\
& X(Y(x, y), z)=U(V(x, z), Z(y, z)) .
\end{aligned}
$$

Перейдем к классификации нетривиальных сверхтождеств левой и правой дистрибутивности, вьполняющихся в нетривиальных $q$-алгебрах. 
ТеОРема 4.2. Если в нетривиальной q-алгебре с тривиальньлм сверхтождеством правой дистрибутивности

$$
X(X(x, y), z)=X(X(x, z), X(y, z))
$$

выполняется нетривиальное сверхтождество левой дистрибутивности, тогда оно будет иметь функииональный ранг 2 и вид $\left(d_{1}\right)$. Если в нетривиальной q-алгебре с тривиальным сверхтождеством левой дистрибутивности

$$
X(x, X(y, z))=X(X(x, y), X(x, z))
$$

выполняется нетривиальное сверхтохсдество правой дистрибутивности, тогда оно будет иметь функциональньи ианг 2 и вид $\left(d_{2}\right){ }^{8}$

ДокАЗАТЕльство. Поскольку в сверхтождестве левой (правой) дистрибутивности имеется всего пять мест для функциональных переменных, то согласно лемме 4.1 функциональный ранг нетривиального сверхтождества левой (правой) дистрибутивности не может превышать 2 , иначе в сверхтождестве существовала бы сингулярная функциональная переменная. Таким образом, функциональный ранг должен быть равен двум. Поэтому одна из функциональных переменных должна повторяться два раза, а другая - три раза. Помимо сверхтождества $\left(d_{1}\right)$ имеется еше девять таких сверхтождеств левой дистрибутивности:

$$
\begin{aligned}
& X(x, X(y, z))=Y(Y(x, y), Y(x, z)), \\
& X(x, X(y, z))=Y(X(x, y), Y(x, z)), \\
& X(x, X(y, z))=Y(Y(x, y), X(x, z)), \\
& X(x, X(y, z))=X(Y(x, y), Y(x, z)), \\
& X(x, Y(y, z))=X(Y(x, y), Y(x, z)), \\
& X(x, Y(y, z))=X(Y(x, y), X(x, z)), \\
& X(x, Y(y, z))=X(X(x, y), Y(x, z)), \\
& X(x, Y(y, z))=Y(Y(x, y), X(x, z)), \\
& X(x, Y(y, z))=Y(X(x, y), Y(x, z)) .
\end{aligned}
$$

Требуется доказать, что ни одно из сверхтождеств (4.3)-(4.11) не может вьполняться ни в какой нетривиальной $q$-алгебре с тривиальным сверхтождеством правой дистрибутивности (4.1).

Пусть в нетривиальной $q$-алгебре $(Q ; \Sigma)$ со сверхтождеством $(4.1)$ вьполняется сверхтождество (4.3). Согласно определению $q$-алгебры существует такая операция $A \in \Sigma$, что $Q(A)$ - квазигруппа, следовательно, $Q(A)$ - дистрибутивная квазигруппа, т.е. для любых $x, y, z \in Q$ справедливы равенства:

$$
\begin{aligned}
& A(x, A(y, z))=A(A(x, y), A(x, z)), \\
& A(A(x, y), z)=A(A(x, z), A(y, z)) .
\end{aligned}
$$

\footnotetext{
${ }^{8} \mathrm{O}$ сверхтождествах $\left(d_{1}\right),\left(d_{2}\right)$ см. в $\S 1$.
} 
Из первого равенства при $y=x$ и после сокращения следует $A(x, x)=x$ для любого $x \in Q$, т.е. операция $A$ идемпотентна.

На множестве $Q$ определим операцию (о):

$$
x \circ y=A\left(R_{e}^{-1} x, L_{e}^{-1} x\right), \quad e \in Q,
$$

где $R_{e}(x)=A(x, e), L_{e}(x)=A(e, x)$. Получаем лупу $Q($ о $)$ с единицей $e=A(e, e)$. Следовательно,

$$
A(x, y)=R_{e}(x) \circ L_{e}(x),
$$

где $R_{e}, L_{e} \in \operatorname{Aut} Q(\circ)$. Действительно,

$$
\begin{aligned}
R_{e}(x \circ y) & =R_{e} A\left(R_{e}^{-1} x, L_{e}^{-1} y\right)=A\left(A\left(R_{e}^{-1} x, L_{e}^{-1} y\right), e\right) \\
& =A\left(A\left(R_{e}^{-1} x, e\right), A\left(L_{e}^{-1} y, e\right)\right)=A\left(R_{e}\left(R_{e}^{-1} x\right), R_{e}\left(L_{e}^{-1} y\right)\right) \\
& =A\left(R_{e}^{-1}\left(R_{e} x\right), L_{e}^{-1}\left(R_{e} y\right)\right)=R_{e}(x) \circ R_{e}(y),
\end{aligned}
$$

так как $R_{e} L_{e}=L_{e} R_{e}$, поскольку

$$
R_{e}\left(L_{e} x\right)=A(A(e, x), e)=A(A(e, e), A(x, e))=A(e, A(x, e))=L_{e}\left(R_{e} x\right) .
$$

Аналогично доказьвается, что $L_{e}$ - автоморфизм лупы $Q\left(\right.$ о). Обозначая $R_{e}=\varphi$, $L_{e}=\psi$, приходим к равенству:

$$
A(x, y)=\varphi x \circ \psi y, \quad \varphi, \psi \in \operatorname{Aut} Q(\circ),
$$

где $A \in \Sigma, Q(A)$ - квазигруппа.

В сверхтождестве (4.3), полагая $X=A_{i} \in \Sigma$ и $Y=A$, получим

$$
\varphi(\varphi x \circ \psi y) \circ \psi(\varphi x \circ \psi z)=A_{i}\left(x, A_{i}(y, z)\right),
$$

откуда при $x=e$ следует

$$
\varphi(\psi y) \circ \psi^{2}(z)=\mu_{i} A_{i}(y, z),
$$

где $\mu_{i}(x)=A_{i}(e, x)$. Далее:

$$
\begin{gathered}
\mu_{i} A_{i}(e, z)=\psi^{2}(z), \\
\mu_{i}^{2}(z)=\psi^{2}(z) .
\end{gathered}
$$

Из последнего равенства вытекает, что $\mu_{i}^{2}$ - подстановка, следовательно, $\mu_{i}-$ подстановка и $Q\left(A_{i}\right)$ - квазигруппа (изотопная лупе $Q\left(\right.$ о ) ) для любого $A_{i} \in \Sigma$, т.е. $(Q ; \Sigma)$ - обратимая алгебра. Следовательно, $(Q ; \Sigma)$ - идемпотентная алгебра, т.е. в ней выполняется и сверхтождество идемпотентности $X(x, x)=x$.

Пусть $A_{1}, A_{2} \in \Sigma$ и $A_{1} \neq A_{2}$. Полагая в сверхтождестве (4.3) $X=A_{1}, Y=A_{2}$, $y=z$, приходим к противоречию:

$$
\begin{gathered}
A_{1}\left(x, A_{1}(y, y)\right)=A_{2}\left(A_{2}(x, y), A_{2}(x, y)\right), \\
A_{1}(x, y)=A_{2}(x, y), \\
A_{1}=A_{2} .
\end{gathered}
$$

Аналогично рассматриваются и случаи сверхтождеств (4.4)-(4.11). Вторая часть теоремы доказьвается подобными рассуждениями. 
СлЕДСТВИЕ 4.3 [10], [58]. Если в нетривиальной обратимой алгебре выполняется нетривиальное сверхтождество левой (правой) дистрибутивности, тогда оно будет функиионального ранга 2 и вида $\left(d_{1}\right)$ (соответственно $\left.\left(d_{2}\right)\right)$.

ДокАЗАТЕЛЬство. Идемпотентность обратимой алгебры со сверхтождеством левой (правой) дистрибутивности сразу следует из сократимости в обратимых алгебрах. Далее, в идемпотентных сократимых алгебрах из любого сверхтождества (4.3)-(4.11) вытекает сверхтождество $X(x, y)=Y(x, y)$.

СлЕДСТВИЕ 4.4 [8], [9]. Если в нетривиальной q-алгебре выполняются нетривиальные сверхтожсдества левой и правой дистрибутивности, тогда сверхтождество левой (правой) дистрибутивности будет функиионального ранга 2 и вида $\left(d_{1}\right)$ (соответственно $\left.\left(d_{2}\right)\right)$.

О сверхтождествах дистрибутивности см. также [59], [60].

Tеорема 4.3. В обратимой алгебре $(Q ; \Sigma)$ сверхтождества $\left(d_{1}\right)$ и (4.1) вьполняются тогда и только тогда, когда существует коммутативная лупа Муфанг $Q($ (о) такая, что каждая операция $A \in \Sigma$ определяется по правилу:

$$
A(x, y)=\varphi_{A}(x) \circ \psi_{A}(y)
$$

где $\varphi_{A}, \psi_{A} \in \operatorname{Aut} Q(\circ), \varphi_{A} \in \operatorname{Aut} Q(A),\left(\psi_{A}, \widetilde{\varepsilon}\right) \in \operatorname{Aut}(Q ; \Sigma), \varphi_{A}(x) \circ \psi_{A}(x)=x$, $x \circ \varphi_{A}(x) \in K_{Q}\left(\right.$ ядро ${ }^{9}$ лупьи $\left.Q(\circ)\right)$ для всех $A \in \Sigma, x, y \in Q$.

ДокАЗАТЕЛЬСТво. Достаточность. Поскольку $x \circ \varphi_{B}(x) \in K_{Q}$, то, заменяя здесь $x$ на $\varphi_{B}^{-1}(x)$, получим

$$
\varphi_{B}^{-1}(x) \circ x=x \circ \varphi_{B}^{-1}(x) \in K_{Q} .
$$

С другой стороны в силу биассоциативности луп Муфанг

$$
x \circ \varphi_{B}^{-1}(x)=x^{3} \circ\left(x^{-2} \circ \varphi_{B}^{-1}(x)\right),
$$

откуда с учетом свойства $x^{3} \in K_{Q}$ для коммутативных луп Муфанг [61] вьводим:

$$
\begin{gathered}
x^{-2} \circ \varphi_{B}^{-1}(x) \in K_{Q}, \\
x^{2} \circ\left(\varphi_{B}^{-1}(x)\right)^{-1} \in K_{Q}
\end{gathered}
$$

для любых $x \in Q, B \in \Sigma$.

По условию, $Q($ о $)$ - коммутативная лупа Муфанг, т.е. в $Q($ (о) справедливо тождество $x^{2} \circ(y \circ z)=(x \circ y) \circ(x \circ z)$.

\footnotetext{
${ }^{9}$ Левым (правым, средним) ядром лупы $Q(\cdot)$ называется совокупность всех элементов $a \in Q$, для которых $a x \cdot y=a \cdot x y$ (соответственно $x y \cdot a=x \cdot y a, x a \cdot y=x \cdot a y)$ при любых $x, y \in Q$. Ядром лупы называется пересечение всех трех ее ядер. В лупах Муфанг все три ядра совпадают, более того, в лупах со свойством обратимости (т.е. в $I P$-лупах) все три ядра совпадают [61]. Лупа $Q(\cdot)$ назьвается лупой Муфанг, если она удовлетворяет тождеству $x(y z \cdot x)=x y \cdot z x$. Коммутативные лупы Муфанг характеризуются одним тождеством: $x^{2} \cdot y z=x y \cdot x z$.
} 
Однако, с учетом свойства $x^{2} \circ\left(\varphi_{B}^{-1}(x)\right)^{-1} \in K_{Q}$ имеем:

$$
\begin{aligned}
\left(\varphi_{B}^{-1}(x) \circ(y \circ z)\right) \circ\left(x^{2} \circ\left(\varphi_{B}^{-1}(x)\right)^{-1}\right) & =\left(x^{2} \circ\left(\varphi_{B}^{-1}(x)\right)^{-1}\right) \circ\left(\varphi_{B}^{-1}(x) \circ(y \circ z)\right) \\
& =\left(\left(x^{2} \circ\left(\varphi_{B}^{-1}(x)\right)^{-1}\right) \circ \varphi_{B}^{-1}(x)\right) \circ(y \circ z) \\
& =x^{2} \circ(y \circ z)
\end{aligned}
$$

и, аналогично,

$$
(x \circ y) \circ(x \circ z)=\left((x \circ y) \circ\left(\left(x^{-1} \circ \varphi_{B}^{-1}(x)\right) \circ z\right)\right) \circ\left(x^{2} \circ\left(\varphi_{B}^{-1}(x)\right)^{-1}\right) .
$$

Следовательно,

$$
\begin{gathered}
\varphi_{B}^{-1}(x) \circ(y \circ z)=(x \circ y) \circ\left(\left(x^{-1} \circ \varphi_{B}^{-1}(x)\right) \circ z\right), \\
\varphi_{B}\left(\varphi_{B}^{-1}(x) \circ(y \circ z)\right)=\varphi_{B}\left((x \circ y) \circ\left(\left(x^{-1} \circ \varphi_{B}^{-1}(x)\right) \circ z\right)\right), \\
x \circ \varphi_{B}(y \circ z)=\varphi_{B}(x \circ y) \circ\left(\left(\varphi_{B}\left(x^{-1}\right) \circ x\right) \circ \varphi_{B}(z)\right), \\
x \circ\left(\varphi_{B}(y) \circ \varphi_{B}(z)\right)=\left(\varphi_{B} x \circ \varphi_{B} y\right) \circ\left(\psi_{B} x \circ \varphi_{B} z\right), \\
x \circ(y \circ z)=\left(\varphi_{B}(x) \circ y\right) \circ\left(\psi_{B}(x) \circ z\right)
\end{gathered}
$$

для любых $x, y, z \in Q, B \in \Sigma$. Следовательно, для любого $B \in \Sigma$ имеем:

$$
\begin{aligned}
x \circ B(y, z) & =x \circ\left(\varphi_{B} y \circ \psi_{B} z\right) \stackrel{(4.12)}{=}\left(\varphi_{B} x \circ \varphi_{B} y\right) \circ\left(\psi_{B} x \circ \psi_{B} z\right) \\
& =\varphi_{B}(x \circ y) \circ \psi_{B}(x \circ z)=B(x \circ y, x \circ z) .
\end{aligned}
$$

Теперь сверхтождества $\left(d_{1}\right)$ и (4.1) легко проверяются:

$$
\begin{aligned}
& B(A(x, y), A(x, z))= B\left(\varphi_{A} x \circ \psi_{A} y, \varphi_{A} x \circ \psi_{A} z\right) \\
&= \varphi_{A} x \circ B\left(\psi_{A} y, \psi_{A} z\right)=\varphi_{A} x \circ \psi_{A} B(y, z)=A(x, B(y, z)) ; \\
& \begin{aligned}
A(A(x, z), A(y, z)) & =A\left(\varphi_{A} x \circ \psi_{A} z, \varphi_{A} y \circ \psi_{A} z\right) \\
& =A\left(\psi_{A} z \circ \varphi_{A} x, \psi_{A} z \circ \varphi_{A} y\right)=\psi_{A} z \circ A\left(\varphi_{A} x, \varphi_{A} y\right) \\
& =\psi_{A} z \circ \varphi_{A} A(x, y)=\varphi_{A} A(x, y) \circ \psi_{A} z=A(A(x, y), z) .
\end{aligned}
\end{aligned}
$$

Необходимость. Пусть $(Q ; \Sigma)$ - обратимая алгебра со сверхтождествами $\left(d_{1}\right),(4.1)$ и $e$-произвольньй фиксированньй элемент из $Q$. Очевидно, что $(Q ; \Sigma)$ - идемпотентна. Для любого $A \in \Sigma$ рассмотрим отображения (биекции)

$$
\begin{aligned}
R_{A}: x \rightarrow A(x, e), & x \in Q, \\
L_{A}: x \rightarrow A(e, x), & x \in Q,
\end{aligned}
$$

и определим операцию оА следуюшим образом:

$$
u \circ_{\mathrm{A}} v=A\left(R_{A}^{-1}(u), L_{A}^{-1}(v)\right), \quad u, v \in Q .
$$


Легко заметить, что $Q\left({ }_{\mathrm{A}}\right)$ - лупа (для любого $A \in \Sigma$ ) с единицей $e=A(e, e)$. Поскольку $A(u, v)=R_{A}(u) \circ_{\mathrm{A}} L_{A}(v)$, то $u=A(u, u)=R_{A}(u) \circ_{\mathrm{A}} L_{A}(u)$. Далее:

$$
\begin{aligned}
R_{A}\left(L_{B}(u)\right) & =R_{A} B(e, u)=A(B(e, u), e) \\
& =A(B(e, u), B(e, e))=B(e, A(u, e))=L_{B}\left(R_{A}(u)\right) ; \\
L_{A}\left(L_{B}(u)\right) & =L_{A} B(e, u)=A(e, B(e, u)) \\
& =B(A(e, e), A(e, u))=B(e, A(e, u))=L_{B}\left(L_{A}(u)\right) .
\end{aligned}
$$

Таким образом, для любых $A, B \in \Sigma$ имеем:

$$
R_{A} L_{B}=L_{B} R_{A}, \quad L_{A} L_{B}=L_{B} L_{A} .
$$

Кроме того, из сверхтождества $\left(d_{1}\right)$ вытекает,что $L_{A}$ - автоморфизм алгебры $(Q ; \Sigma)$, а из сверхтождества $(4.1)$ следует, что $R_{A}$ - автоморфизм квазигруппы $(Q ; A)$ для любого $A \in \Sigma$ :

$$
\begin{aligned}
& L_{A} B(x, y)=A(e, B(x, y))=B(A(e, x), A(e, y))=B\left(L_{A}(x), L_{A}(y)\right) \\
& R_{A} A(x, y)=A(A(x, y), e)=A(A(x, e), A(y, e))=A\left(R_{A}(x), R_{A}(y)\right) .
\end{aligned}
$$

Исходя из вьшесказанного, получаем:

$$
\begin{aligned}
L_{A}\left(x \circ_{\mathrm{B}} y\right) & =L_{A} B\left(R_{B}^{-1} x, L_{B}^{-1} y\right) \\
& =B\left(L_{A}\left(R_{B}^{-1} x\right), L_{A}\left(L_{B}^{-1} y\right)\right)=B\left(R_{B}^{-1}\left(L_{A} x\right), L_{B}^{-1}\left(L_{A} y\right)\right) \\
& =R_{B}\left(R_{B}^{-1}\left(L_{A} x\right)\right) \circ_{\mathrm{A}} L_{B}\left(L_{B}^{-1}\left(L_{A} y\right)\right)=L_{A} x \circ_{\mathrm{A}} L_{A} y
\end{aligned}
$$

и, аналогично,

$$
R_{A}\left(x \circ_{\mathrm{A}} y\right)=R_{A}(x) \circ_{\mathrm{A}} R_{A}(y),
$$

для любых $A, B \in \Sigma$.

Полагая $\varphi_{A}=R_{A}, \psi_{A}=L_{A}$, приходим к равенству:

$$
A(x, y)=\varphi_{A}(x) \circ_{\mathrm{A}} \psi_{A}(y) .
$$

Пусть $\left(\mathrm{o}_{\mathrm{A}}\right)=(\circ), \varphi_{A}=\varphi, \psi_{A}=\psi$. Тогда, исходя из левого дистрибутивного закона

$$
A(x, A(y, z))=A(A(x, y), A(x, z)),
$$

получаем:

$$
\begin{aligned}
\varphi x \circ \psi(\varphi y \circ \psi z) & =\varphi(\varphi x \circ \psi y) \circ \psi(\varphi x \circ \psi z), \\
\varphi x \circ\left(\psi(\varphi y) \circ \psi^{2} z\right) & =\left(\varphi^{2} x \circ \varphi(\psi y)\right) \circ\left(\psi(\varphi x) \circ \psi^{2} z\right) .
\end{aligned}
$$

В последнем равенстве сделаем замены: $x \rightarrow \varphi^{-1} x, y \rightarrow \varphi^{-1}\left(\psi^{-1} y\right), z \rightarrow \psi^{-2} z$; учитьвая равенство $\varphi \psi=\psi \varphi$, вьводим:

$$
x \circ(y \circ z)=(\varphi x \circ y) \circ(\psi x \circ z) .
$$


Пусть $y=e$, тогда

$$
x \circ z=\varphi x \circ(\psi x \circ z),
$$

и, заменяя $z$ на $y \circ(\psi x)^{-1}$, где $s^{-1}$ - правый обратный элемента $s$ в лупе $Q(\circ)$, получаем

$$
x \circ\left(y \circ(\psi x)^{-1}\right)=\varphi x \circ\left(\psi x \circ\left(y \circ(\psi x)^{-1}\right)\right)
$$

или согласно (4.13)

$$
\begin{aligned}
(\varphi x \circ y) \circ\left(\psi x \circ(\psi x)^{-1}\right) & =\varphi x \circ\left(\psi x \circ\left(y \circ(\psi x)^{-1}\right)\right), \\
\varphi x \circ y & =\varphi x \circ\left(\psi x \circ\left(y \circ(\psi x)^{-1}\right)\right), \\
y & =\psi x \circ\left(y \circ(\psi x)^{-1}\right),
\end{aligned}
$$

откуда, заменяя $x$ на $\psi^{-1}(x)$, получаем тождество:

$$
x \circ\left(y \circ x^{-1}\right)=y .
$$

Рассмотрим левый дистрибутивный закон для операции $A \in \Sigma$ и преобразуем его следуюшим образом (с использованием предложения 1.2 в случае $\Sigma=\{A\})$ :

$$
\begin{aligned}
& A(x, A(y, z))=A(A(x, y), A(x, z)), \\
& A(x, A(y, z))=A(A(x, A(x, z)), A(y, A(x, z))), \\
& A(x, A(x, z))={ }^{-1} A(A(x, A(y, z)), A(y, A(x, z))), \\
& A(x, A(x, z))={ }^{-1} A(A(x, A(y, z)), A(A(y, x), A(y, z))), \\
& A(x, A(x, z))=A\left({ }^{-1} A(x, A(y, x)), A(y, z)\right) .
\end{aligned}
$$

Пусть $x=e$ и $\alpha(y)={ }^{-1} A(e, y)$, тогда из последнего равенства имеем:

$$
\begin{aligned}
\psi^{2}(z)=A(\alpha(\varphi(y)), A(y, z)) & =\varphi(\alpha(\varphi y)) \circ \psi(\varphi y \circ \psi z) \\
& =\varphi(\alpha(\varphi y)) \circ\left(\psi(\varphi y) \circ \psi^{2}(z)\right)
\end{aligned}
$$

т.е. $z=\varphi\left(\alpha\left(\psi^{-1}(y)\right)\right) \circ(y \circ z)=I(y) \circ(y \circ z)$, где $I: Q \rightarrow Q-$ подстановка и, очевидно, $I(y)=y^{-1}$. Таким образом, справедливо тождество

$$
x^{-1} \circ(x \circ y)=y,
$$

причем $\left(x^{-1}\right)^{-1}=x$, поскольку согласно $(4.15)$

$$
x \circ y=\left(x^{-1}\right)^{-1} \circ\left(x^{-1} \circ(x \circ y)\right)=\left(x^{-1}\right)^{-1} \circ y .
$$

Сравнивая тождества (4.14) и (4.15), находим $y \circ x^{-1}=x^{-1} \circ y$, т.е. лупа $Q(\circ)-$ коммутативная лупа со свойством обратимости.

Заменяя в тождестве (4.13) $x$ на $\varphi^{-1} x$ и учитывая равенство

$$
\varphi^{-1} x=A\left(\varphi^{-1} x, \varphi^{-1} x\right)=\varphi\left(\varphi^{-1} x\right) \circ \psi\left(\varphi^{-1} x\right)=x \circ \psi\left(\varphi^{-1} x\right),
$$


получим

$$
\left(x \circ \psi\left(\varphi^{-1} x\right)\right) \circ(y \circ z)=(x \circ y) \circ\left(\psi\left(\varphi^{-1} x\right) \circ z\right),
$$

T.e.

$$
(x \circ f(x)) \circ(y \circ z)=(x \circ y) \circ(f(x) \circ z),
$$

где $f(x)=\psi\left(\varphi^{-1} x\right)$. Поэтому

$$
(x \circ y) \circ(f(x) \circ z)=(x \circ f(x)) \circ(z \circ y)=(x \circ z) \circ(f(x) \circ y),
$$

т.е.

$$
\begin{aligned}
& (x \circ y) \circ L_{f(x)}(z)=(x \circ z) \circ L_{f(x)}(y), \\
& \left(x \circ L_{f(x)}^{-1}(y)\right) \circ z=\left(x \circ L_{f(x)}^{-1}(z)\right) \circ y, \\
& L_{x}\left(L_{f(x)}^{-1}(y)\right) \circ z=L_{x}\left(L_{f(x)}^{-1}(z)\right) \circ y,
\end{aligned}
$$

где $L_{a}(z)=a \circ z, L_{a}\left(L_{a}^{-1}(z)\right)=a \circ L_{a}^{-1}(z), z=a \circ L_{a}^{-1}(z), a^{-1} \circ z=a^{-1}\left(a \circ L_{a}^{-1}(z)\right)$, $a^{-1} \circ z=L_{a}^{-1}(z)$. Следовательно,

$$
\begin{aligned}
\left(u \circ L_{x}\left(L_{f(x)}^{-1}(e)\right)\right) \circ v & =\left(e \circ L_{x}\left(L_{f(x)}^{-1}(u)\right)\right) \circ v \\
& =L_{x}\left(L_{f(x)}^{-1}(u)\right) \circ v=u \circ L_{x}\left(L_{f(x)}^{-1}(v)\right) \\
& =u \circ\left(e \circ L_{x}\left(L_{f(x)}^{-1} v\right)\right)=u \circ\left(L_{x}\left(L_{f(x)}^{-1} e\right) \circ v\right),
\end{aligned}
$$

т.е.

$$
L_{x}\left(L_{f(x)}^{-1} e\right)=x \circ\left(f(x)^{-1} \circ e\right)=x \circ f(x)^{-1} \in K_{Q}
$$

(в лупах со свойством обратимости все три ядра совпадают).

Исходя из доказанного включения и равенства (4.16), легко проверить муфанговость коммутативной лупы $Q(\circ)$ :

$$
\begin{aligned}
x^{2} \circ(y \circ z) & =(y \circ z) \circ x^{2} \\
& =(y \circ z) \circ\left((x \circ x) \circ\left(f(x) \circ f(x)^{-1}\right)\right) \\
& =(y \circ z) \circ\left((x \circ f(x)) \circ\left(x \circ f(x)^{-1}\right)\right) \\
& =((y \circ z) \circ(x \circ f(x))) \circ\left(x \circ f(x)^{-1}\right) \\
& =(x \circ f(x)) \circ(y \circ z) \circ\left(x \circ f(x)^{-1}\right) \\
& =((x \circ y) \circ(f(x) \circ z)) \circ\left(x \circ f(x)^{-1}\right) \\
& =(x \circ y) \circ\left((f(x) \circ z) \circ\left(x \circ f(x)^{-1}\right)\right) \\
& =(x \circ y) \circ\left((z \circ f(x)) \circ\left(x \circ f(x)^{-1}\right)\right) \\
& =(x \circ y) \circ\left(z \circ\left(f(x) \circ\left(f(x)^{-1} \circ x\right)\right)\right) \\
& =(x \circ y) \circ(z \circ x)=(x \circ y) \circ(x \circ z) .
\end{aligned}
$$


Докажем включение $x \circ \varphi(x) \in K_{Q}$. Исходя из равенства (4.13), получим:

$$
\begin{aligned}
\left(x \circ\left(x^{-1} \circ \varphi^{-1}(x)\right)\right) \circ(y \circ z) & =\varphi^{-1} x \circ(y \circ z)=(x \circ y) \circ\left(\psi\left(\varphi^{-1} x\right) \circ z\right) \\
& =(x \circ y) \circ\left(\left(\varphi^{-1} x \circ x^{-1}\right) \circ z\right) \\
& =(x \circ y) \circ\left(\left(x^{-1} \circ \varphi^{-1} x\right) \circ z\right),
\end{aligned}
$$

поскольку $\psi(x)=x \circ \varphi\left(x^{-1}\right)$. Таким образом, мы пришли к равенству вида $(4.16) \mathrm{c}$ функцией $f(x)=x^{-1} \circ \varphi^{-1}(x)$. Согласно (4.17)

$$
\begin{gathered}
x^{-1} \circ f(x) \in K_{Q}, \\
x^{-1} \circ\left(x^{-1} \circ \varphi^{-1}(x)\right)=x^{-2} \circ \varphi^{-1}(x) \in K_{Q} .
\end{gathered}
$$

Однако $x^{3} \in K_{Q}$ и, очевидно, $K_{Q}$ инвариантно относительно автоморфизма $\varphi$, поэтому $x^{3} \circ\left(x^{-2} \circ \varphi^{-1} x\right) \in K_{Q}, x \circ \varphi^{-1}(x) \in K_{Q}, \varphi(x) \circ x \in K_{Q}$.

Для завершения доказательства теоремы 4.3 остается показать, что в обратимой алгебре $(Q ; \Sigma)$ со сверхтождествами $\left(d_{1}\right),(4.1)$ справедливо равенство оА $=$ ов для любых $A, B \in \Sigma$.

Лемма 4.2. Если $(Q ; \Sigma)$ - обратимая алгебра со сверхтождеством $\left(d_{1}\right)$, тогда для любых $A, B \in \Sigma$ справедливо тождество

$$
x \circ_{\mathrm{A}} B(y, z)=B\left(x \circ{ }_{\mathrm{A}} y, x \circ{ }_{\mathrm{A}} z\right) .
$$

ДокАЗАТЕЛЬСтво. Согласно сверхтождеству $\left(d_{1}\right)$ имеем

$$
A(x, B(y, z))=B(A(x, y), A(x, z)),
$$

поэтому

$$
R_{A} x \circ_{\mathrm{A}} L_{A} B(y, z)=B\left(R_{A} x \circ_{\mathrm{A}} L_{A} y, R_{A} x \circ_{\mathrm{A}} L_{A} z\right) .
$$

Поскольку $L_{A}-$ автоморфизм алгебры $(Q ; \Sigma)$ и лупы $Q($ оA $)$, то этим же свойством обладает и подстановка $L_{A}^{-1}$ :

$$
\begin{gathered}
L_{A}^{-1}\left(R_{A} x \circ_{\mathrm{A}} L_{A} B(y, z)\right)=L_{A}^{-1} B\left(R_{A} x \circ_{\mathrm{A}} L_{A} y, R_{A} x \circ_{\mathrm{A}} L_{A} z\right), \\
L_{A}^{-1}\left(R_{A} x\right) \circ_{\mathrm{A}} B(y, z)=B\left(L_{A}^{-1}\left(R_{A} x\right) \circ_{\mathrm{A}} y, L_{A}^{-1}\left(R_{A} x\right) \circ_{\mathrm{A}} z\right),
\end{gathered}
$$

т.e.

$$
x \circ_{\mathrm{A}} B(y, z)=B\left(x \circ \circ_{\mathrm{A}} y, x \circ \circ_{\mathrm{A}} z\right) .
$$

Лемма 4.3. Пусть $(Q ; \Sigma)$ - обратимая алгебра со сверхтожсдествами $\left(d_{1}\right)$ $u$ (4.1), и пусть $A \in \Sigma,\left(\mathrm{o}_{\mathrm{A}}\right)=($ (о). Тогда подлупа лупьи $Q(\mathrm{\circ})$, порожденная әлементами $x, y, L_{B}(x)$, для любых $x, y \in Q, B \in \Sigma$, будет әруппой. 
ДокАЗАТЕЛЬСТво. Согласно равенству (4.18)

$$
x \circ B(y, z)=B(x \circ y, x \circ z),
$$

откуда при $x=z^{-1}$ (берется обратный элемент в лупе $Q($ о)) получаем

$$
\begin{gathered}
z^{-1} \circ B(y, z)=B\left(z^{-1} \circ y, e\right)=R_{B}\left(y \circ z^{-1}\right), \\
B(y, z)=R_{B}\left(y \circ z^{-1}\right) \circ z .
\end{gathered}
$$

Аналогично, при $x=y^{-1}$ имеем:

$$
B(y, z)=L_{B}\left(z \circ y^{-1}\right) \circ y .
$$

Следовательно,

$$
R_{B}\left(y \circ z^{-1}\right) \circ z=L_{B}\left(z \circ y^{-1}\right) \circ y .
$$

Полагая здесь $y=e$ и заменяя $z$ на $z \circ y^{-1}=x$, получим:

$$
\begin{gathered}
R_{B}\left(y \circ z^{-1}\right) \circ\left(z \circ y^{-1}\right)=L_{B}\left(z \circ y^{-1}\right), \\
R_{B}\left(y \circ z^{-1}\right)=L_{B}\left(z \circ y^{-1}\right) \circ\left(z \circ y^{-1}\right)^{-1}, \\
R_{B}\left(y \circ z^{-1}\right) \circ z=\left(L_{B}\left(z \circ y^{-1}\right) \circ\left(z \circ y^{-1}\right)^{-1}\right) \circ z, \\
L_{B}\left(z \circ y^{-1}\right) \circ y=\left(L_{B}\left(z \circ y^{-1}\right) \circ\left(z \circ y^{-1}\right)^{-1}\right) \circ z, \\
L_{B}(x) \circ\left(x^{-1} \circ z\right)=\left(L_{B}(x) \circ x^{-1}\right) \circ z,
\end{gathered}
$$

т.е. для элементов $L_{B}(x), x^{-1}, z$ вьполняется соотношение ассоциативности. Поэтому согласно теореме Муфанг [37], если в лупе Муфанг $Q$ (о) для трех ее элементов $a, b, c$ вьполняется равенство $a \circ(b \circ c)=(a \circ b) \circ c$, тогда они порождают ассоциативную подлупу, т.е. групшу. Следовательно, и $L_{B}(x), x, z$ порождают ассоциативную подлупу.

СлЕДСТвИЕ 4.5. Если $(Q ; \Sigma)$ - обратимая алгебра со сверхтождествами $\left(d_{1}\right)$ $u(4.1), \operatorname{mогда~}\left(\mathrm{o}_{\mathrm{A}}\right)=\left(\mathrm{o}_{\mathrm{B}}\right)$ для любых $A, B \in \Sigma$.

ДоКАЗАТЕЛЬСТВо. Пусть $($ ов$)=(\oplus),\left({ }_{\mathrm{A}}\right)=($ о $)$. Поскольку $L_{B}-$ автоморфизм лупы $Q($ о), то

$$
B(y, z)=R_{B} y \oplus L_{B} z=L_{B}\left(z \circ y^{-1}\right) \circ y=\left(L_{B}(z) \circ\left(L_{B} y\right)^{-1}\right) \circ y,
$$

откуда при $z=e$ получим $R_{B} y=\left(L_{B} y\right)^{-1} \circ y$. Применяя лемму 4.3 к элементам $y, L_{B} y$ и $L_{B} z$, получим:

$$
\begin{aligned}
R_{B} y \oplus L_{B} z & =\left(L_{B} z \circ\left(L_{B} y\right)^{-1}\right) \circ y=L_{B} z \circ\left(\left(L_{B} y\right)^{-1} \circ y\right) \\
& =L_{B} z \circ R_{B} y=R_{B} y \circ L_{B} z .
\end{aligned}
$$

Теорема 4.3 доказана. 
TеОРема 4.3'. В обратимой алгебре $(Q ; \Sigma)$ выполняются сверхтождества $\left(d_{2}\right)$ и (4.2) тогда и только тогда, когда существует коммутативная лупа Муфанг $Q($ (о) такая, что каждая операчия $A \in \Sigma$ определяется по правилу:

$$
A(x, y)=\varphi_{A}(x) \circ \psi_{A}(y),
$$

где $\varphi_{A}, \psi_{A} \in \operatorname{Aut} Q(\circ), \psi_{A} \in \operatorname{Aut} Q(A),\left(\varphi_{A}, \widetilde{\varepsilon}\right) \in \operatorname{Aut}(Q ; \Sigma), \varphi_{A}(x) \circ \psi_{A}(x)=x$, $x \circ \varphi_{A}(x) \in K_{Q}$ для всех $A \in \Sigma, x, y \in Q$.

СлЕДСТВИЕ 4.6. В обратимой алгебре $(Q ; \Sigma)$ сверхтождества дистрибутивности $\left(d_{1}\right)$ и $\left(d_{2}\right)$ выполняются тогда и только тогда, когда существует коммутативная лупа Муфанг $Q($ () такая, что каждая операчия $A \in \Sigma$ определяется по правилу:

$$
A(x, y)=\varphi_{A}(x) \circ \psi_{A}(y),
$$

где $\varphi_{A}, \psi_{A} \in \operatorname{Aut} Q(\circ),\left(\varphi_{A}, \widetilde{\varepsilon}\right),\left(\psi_{A}, \widetilde{\varepsilon}\right) \in \operatorname{Aut}(Q ; \Sigma), \varphi_{A}(x) \circ \psi_{A}(x)=x, x \circ \varphi_{A}(x) \in$ $K_{Q}$ для всех $A \in \Sigma, x, y \in Q$.

Вопрос характеризации $q$-алгебр со сверхтождествами $\left(d_{1}\right),(4.1)$ (или $\left.\left(d_{2}\right),(4.2)\right)$ остается еще открытым (о характеризации $q$-алгебр со сверхтождествами $\left(d_{1}\right),\left(d_{2}\right)$ см. в [9]). Однако в связи с теоремами 1.1, 1.2 представляет естественный интерес и решение следуюших задач: охарактеризовать обратимые справа (слева) алгебры со сверхтождеством левой (правой) дистрибутивности $\left(d_{1}\right)$ (соответственно $\left.\left(d_{2}\right)\right)$; охарактеризовать обратимые справа (слева) алгебры со сверхтождествами $\left(d_{1}\right)$, $X(x, x)=x$ (соответственно $\left.\left(d_{2}\right), X(x, x)=x\right)$.

В заключение сформулируем ряд результатов вытекающих из теорем $4.3,4.3^{\prime}$ и следствия 4.6 (с использованием предложения 1.2).

СЛЕДСТВИЕ 4.7. Если в обратимой алгебре $(Q ; \Sigma)$ выполняются сверхтождества $\left(d_{1}\right),(4.1)$ (или $\left(d_{2}\right),(4.2)$, или $\left.\left(d_{1}\right),\left(d_{2}\right)\right)$, тогда и в алгебре $(Q ; \Sigma \cup$ $\left.\Sigma^{-1} \cup^{-1} \Sigma\right)$ выполняются әти сверхтождества.

СлЕДСТВИЕ 4.8. Если в обратимой алгебре $(Q ; \Sigma)$ выполняются сверхтождества $\left(d_{1}\right),(4.1)$ (или $\left(d_{2}\right),(4.2)$, или $\left.\left(d_{1}\right),\left(d_{2}\right)\right)$, тогда и в алгебре $\left(Q ; \Sigma \cup \Sigma^{-1} \cup^{-1}\right.$ $\left.\Sigma \cup^{-1}\left(\Sigma^{-1}\right) \cup\left({ }^{-1} \Sigma\right)^{-1} \cup \Sigma^{*}\right)$ выполняются эти сверхтождества.

СледСТВИЕ 4.9. Обратимая алгебра со сверхтождествами $\left(d_{1}\right),(4.1)$ будет абелевой тогда и только тогда, когда она удовлетворяет сверхтождеству $\left(d_{2}\right)$, а соответствующая коммутативная лупа Муфанг (из теоремь 4.3) удовлетворяет тождеству ассоциативности, т.е. является коммутативной группой.

СлЕДСТВИЕ 4.9' . Обратимая алгебра со сверхтождествами $\left(d_{2}\right),(4.2)$ будет абелевой тогда и только тогда, когда она удовлетворяет сверхтохсдеству $\left(d_{1}\right)$, а соответствующая коммутативная лупа Муфанг (из теоремь 4.3') удовлетворяет тождеству ассоциативности.

СлЕДСТВИЕ 4.10. Обратимая алгебра $(Q ; \Sigma)$ со сверхтождествами дистрибутивности $\left(d_{1}\right),\left(d_{2}\right)$ будет абелевой тогда и только тогда, когда в ней выполняется тождество

$$
A(B(x, y), B(z, u))=B(A(x, z), A(y, u))
$$

для некоторых $A, B \in \Sigma$. 
СлЕДСТВИЕ 4.11. Если в обратимой алгебре $(Q ; \Sigma)$ со сверхтождествами дистрибутивности $\left(d_{1}\right),\left(d_{2}\right)$ выполняется соотношение

$$
A(B(a, b), B(c, d))=B(A(a, c), A(b, d))
$$

для некоторых $a, b, c, d \in Q, A, B \in \Sigma$, тогда обратимая подалгебра алгебры $(Q ; \Sigma)$, порожденная әлементами $a, b, c, d$, будет абелевой.

СлЕДСТВИЕ 4.12. В обратимой алгебре со сверхтождествами дистрибутивности $\left(d_{1}\right),\left(d_{2}\right)$ обратимая подалгебра, порожденная любыми тремя ее элементами, абелева.

Об обобщениях сверхтождеств см. в [62]-[67].

\section{СПИСОК ЛИТЕРАТУРЫ}

[1] Чёрч А. Введение в математическую логику. Т. 1. М.: ИЛ, 1961.

[2] Мальцев А. И. Алгебраические системы. М.: Наука, 1970.

[3] Мальцев А.И. Некоторые вопросы теории классов моделей // Труды IV Всесоюзного математического съезда. Т. 1, 1963. С. 169-198.

[4] Кейслер Г., Чэн Ч. Теория моделей. М.: Мир, 1977.

[5] Булос Дж., Джеффрри Р. Вычислимость и логика. М.: Мир, 1994.

[6] Мальцев А. И. Модельные соответствия // Изв. АН СССР. Сер. матем. 1959. Т. 23. № 3. C. 313-336.

[7] Henkin L. Some interconnections between modern algebra and mathematical logic // Trans. Amer. Math. Soc. 1953. V. 74. № 3. P. 410-427.

[8] Мовсисян Ю. М. Введение в теорию алгебр со сверхтождествами. Ереван: Изд-во ЕГУ, 1986.

[9] Мовсисян Ю.М. Сверхтождества и сверхмногообразия в алгебрах. Ереван: Изд-во ЕГУ, 1990.

[10] Белоусов В. Д. Системы квазигрупп с обобщенными тождествами // УМН. 1965. Т. 20. № 1. С. $75-146$.

[11] Мовсисян Ю. М.К теореме Шауфлера // Матем. заметки. 1993. Т. 53. № 2. С. 84-93.

[12] Schauffler R. Die Assoziativität im Ganzen besonders bei Quasigruppen // Math. Z. 1957. V. 67. № 5. P. 428-435.

[13] Denes J., Keedwell A. D. Lattin Squares and Their Applications. Budapest: Academiai Kiado, 1974.

[14] Taylor W. Hyperidentities and hypervarieties // Aequationes Math. 1981. V. 23. P. 30-49.

[15] Neumann W. D. Mal'cev conditions, spectra and Kronecker product // J. Austral. Math. Soc. 1978. V. 25. P. 103-117.

[16] Schröder E. Über eine eigenthümliche Bestimmung einer Function durch formale Anforderungen // J. Reine Angew. Math. 1881. V. 90. P. 189-220.

[17] Schröder E. Lehrbuch der Aritmetik und Algebra. Leipzig, 1873.

[18] Мовсисян Ю. М. Мултипликативная группа поля и сверхтождества // Изв. АН СССР. Сер. матем. 1989. Т. 53. № 5. С. $1040-1055$.

[19] Senft J.R. On weak automorphisms of universal algebras // Dissertationes Math., Rozprawy Mat. V. 74, 1970.

[20] Sichler J. Weak automorphisms of universal algebras // Algebra Universalis. 1973. V. 3. P. 1-7.

[21] Мовсисян Ю. М. Алгебры со сверхтождествами многообразия булевых алгебр // Изв. АН. Сер. матем. 1996. Т. 60. №6. С. 127-168.

[22] Мовсисян Ю. М. Свертождества булевых алгебр // Изв. АН. Сер. матем. 1992. Т. 56. № 3. С. $654-672$. 
[23] Moufang R. Zur structure von Alternativ Körpern // Math. Ann. 1935. V. 110. P. 416-430.

[24] Denecke K., Lau D., Pöschel R., Schweigert D. Free clones and solid varieties // General Algebra and Discrete Mathematics. V. 21. Lemgo: Heldermann-Verlag. Res. Exposition Math., 1995. P. 59-81.

[25] Graczynska E., Schweigert D. Hyperidentities of a given type // Algebra Universalis. 1990. V. 27. P. 305-318.

[26] Холл М. Теория групп. М.: ИЛ, 1962.

[27] Dicker R. M. A set of independent axioms for a field and a condition for a group to be the multiplicative group of a field // Proc. London Math. Soc. 1968. V. 18. P. 114-124.

[28] Grätzer G. A theorem on doubly transitive permutation groups with application to universal algebras // Fund. Math. 1963. V. 53. P. 25-41.

[29] Скорняков Л. А. Стохастическая алгебра // Изв. вузов. Матем. 1985. № 7. С. 3-11.

[30] Romanowska A. B., Smith J. D. H. Modal Theory. Berlin: Heldermann-Verlag, 1985.

[31] Neuman W.D. On the quasivariety on convex subsets of affine spaces // Arch. Math. 1970. V. 21. P. 11-16.

[32] Higgins P. J. Groups with multiple operators // Proc. London Math. Soc. 1956. V. 6. P. $366-416$.

[33] Segre C. Un nuovo campo di ricerche geometriche // Atti Accad. Sci. Torino Cl. Sci. Fis. Mat. Natur. 1889. V. 25. P. 276-301.

[34] Jacobson N. Normal semi-linear transformations // Amer. J. Math. 1939. V. 61. P. 45-58.

[35] Marczewski E. A general scheme of the notions of independence in mathematics // Bull. Acad. Polon. Sci. Ser. Math. Astron. Phys. 1958. V. 6. P. 731-736.

[36] Goetz A. On weak isomorphisms and weak homomorphisms of abstract algebras // Colloq. Math. 1966. V. 14. P. 163-167.

[37] Traczyk T. Weak isomorphisms of Boolean and Post algebras // Colloq. Math. 1965. V. 13. P. $159-164$

[38] Glazek K. Weak automorphisms of general algebras and related topics // Math. Seminar Notes. 1980. V. 8. P. 1-36.

[39] Биркгоф Г. Теория решеток. М.: Наука, 1984.

[40] Kalman J. A. Subdirect decomposition of distributive quasilattices // Fund. Math. 1971. V. 71. P. 161-163.

[41] Мовсисян Ю.М., Бархударян А.Г. О сверхмногообразии QВ-алгебр // Ученые записки ЕГУ. 1996. № 2. С. 16-24.

[42] Padmanabhan R., Penner P. Bases of hyperidentities of lattices and semilattices // C. R. Math. Rep. Acad. Sci. Canada. 1982. V. 4. P. 9-14.

[43] Padmanabhan R., Penner P. Binary hyperidentities of lattices // Aequationes Math. 1992. V. 44. P. 154-167.

[44] Bergman G. M. Hyperidentities of groups and semigroups // Aequationes Math. 1981. V. 23. P. 50-65.

[45] Slominski J. On the satisfiabilities and varieties for abstract algebras induced by the cones and functor dinamics // Demonstratio Math. 1993. V. 26. №1. P. 11-22.

[46] Polak L. On hyperassociativity // Algebra Universalis. 1996. V. 36. P. 363-378.

[47] Denecke K., Pöschel R. The characterization of primal algebras by hyperidentities // Preprint. Berlin: Karl-Weierstrass-Institut Für Mathematik, 1988.

[48] Mal'cev I. A., Schweigert D. Hyperidentitaten von QZ-algebren // Preprint. Kaiserslautern: Fachbereich Mathematik Universitat Kaiserslautern, 1989.

[49] Graczynska E. Connections between identities and hyperidentities // Bull. Sect. Logik Univ. Lódź. 1988. V. 17. P. 34-41.

[50] Penner P. Hyperidentities of semilattices // Houston J. Math. 1984. V. 10. P. 81-108.

[51] Plonka J. On hyperidentities of some varieties // General Algebra and Discrete Mathematics. V. 21. Lemgo: Heldermann-Verlag. Res. Exposition Math., 1995. P. 199-213.

[52] Plonka J., Szylicka Z. Proper hypersubstitutions of the join of independent varieties // Discuss. Math. Algebra Stochastic Methods. 1995. V. 15. № 1. P. 127-134. 
[53] Денеке К., Мальцев И.А., Решке М. О разделимости булевых клонов гипертождествами // Сиб. матем. журн. 1995. Т. 36. № 5. С. 1049-1066.

[54] Bruck R. H. Contributions to the theory of loops // Trans. Amer. Math. Soc. 1946. V. 60. P. 245-354.

[55] Albert A. A. Quasigroups. I // Trans. Amer. Math. Soc. 1943. V. 54. P. 507-519.

[56] Sade A. Groupoïdes en relation associative et semigroupes mutuellement associatifs // Ann. Soc. Sci. Bruxelles. Ser. 1. 1960. V. 74. P. 91-99.

[57] Чупона Г. За финитарните операции // Годишен зб. ун-т Ckonje, Природноматем-фак. (1959-1961). T. 12. C. 7-49.

[58] Aczel J. Proof of a theorem on distributive type hyperidentities // Algebra Universalis. 1971. V. 1. P. 1-6.

[59] Чупона Г. За релацијата дистрибутивности мегу алгебарските операции // Годишен зб. ун-т Ckonje, Природноматем-фак. (1956-1958). Т. 9. С. 23-29.

[60] Stein S.K. On the foundation of quasigroups // Trans. Amer. Math. Soc. 1957. V. 85. P. 228-256.

[61] Bruck R. H. A Survey of Binary Systems (3rd printing, corrected). Berlin: Springer-Verlag, 1971.

[62] Мелконян В.Г. Квазитождества и условные сверхтождества дистрибутивных решеток // Докл. АН Армении. 1993. Т. 94. № 4. С. 195-199.

[63] Graczynska E., Pöschel R., Volkov M. V. Solid pseudovarieties // General Algebra and Applications in Discrete Mathematics. Aachen: Shaker-Verlag, 1997. P. 93-100.

[64] Szylicka Z. Weak hyperidentities of varieties // General Algebra and Applications in Discrete Mathematics. Aachen: Shaker-Verlag, 1997. P. 189-203.

[65] Welke D. Hyperidentitäten partieller Algebren // Dissertation, Universität Potsdam, 1996.

[66] Denecke K., Glazek K. $M$-solid varieties and $Q$-free clones // Math. Slovaca. 1996. V. 46. № 5. P. 515-524.

[67] Arworn S., Denecke K. Groupoids of hypersubstitutions and G-solid varieties // General Algebra and Applications in Discrete Mathematics. Aachen: Shaker-Verlag, 1997. P. 5-22. 